University of Redlands

\title{
Implementing GIS for Facilities Management at the California Institute of Technology
}

\author{
Major Individual Project submitted in partial satisfaction of the requirements \\ for the degree of Master of Science in Geographic Information Systems \\ by
}

Anas A. Bingaith

Douglas M. Flewelling, Ph.D., Committee Chair

Mark Kumler, Ph.D.

May, 2010 
Implementing GIS for Facilities Management at the California Institute of Technology

Copyright (C) 2010

by

Anas Bingaith 
The report of Anas A. Bingaith is approved.

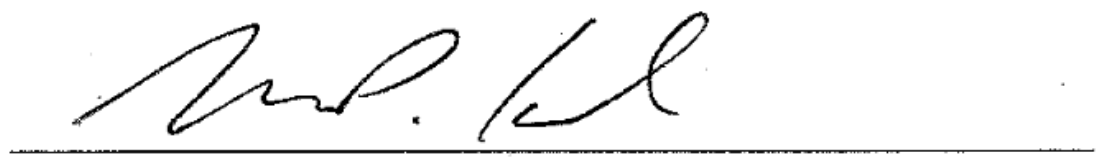

Mark Kumler, Ph.D.

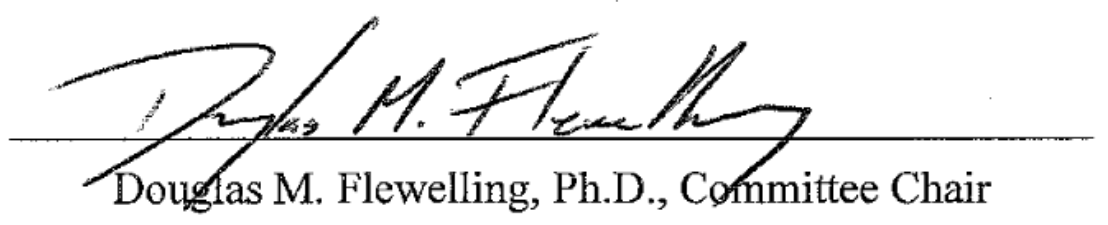

May 2010 



\section{Acknowledgements}

First of all, I would like to thank and praise Allah for giving me the strength and guidance to accomplish the project, and for more blessing than I can reckon.

I would also like to thank gratefully and sincerely my parents who always pray for me, and support me to develop my education and skills to ensure that I have a bright professional future. Thankful words are not enough to express what is in my heart to them.

My education in the USA would not have been possible without the generous full scholarship I received from the Ministry of Higher Education (MOHE) in Saudi Arabia. The Saudi Arabian Cultural Mission (SACM) in the USA also contributes great support, both financially and academically, to help me achieve my academic goal. Many thanks to MOHE and SACM.

I would like to express my deep appreciation to Dr. Douglas Flewelling, my advisor and committee chair, for his guidance, support, and encouragement throughout this project and the program. Dr. Flewelling has always provided me the confidence I needed to accomplish the project.

I also express my gratitude to Dr. Mark Kumler for his accepting to serve on my committee. I am grateful for his valuable feedback and comments in my thesis.

I am greatly indebted to the University of Redlands and the MS GIS program for providing the great environment and services in studying and living. Special thanks go to the MS GIS program coordinator Debra Riley for her constant assistance. Many thanks to John Laska, Brandon Davis, and Ruben Ortiz for the technology support. I extend my appreciation to Mrs. Lynn Flewelling for her amazing effort and skills to help me edit the entire project report. I would also like to acknowledge Mr. Greg Thomas for his great help and the workshop regarding working with CAD files in GIS. Huge thanks to ESRI for allowing me and other students to use the library and borrow needed books and materials. I am also very grateful to all ESRI instructors; in particular, I would like to thank Ray Carnes for sharing his knowledge and expertise. I also thank Don Kuehne, the technical product manager for CAD interoperability at ESRI, for answering my inquiries regarding some issues in the project.

I would like to acknowledge a huge debt to my client Kenneth Hargreaves, the Senior Director of Design and Construction at the California Institute of Technology (Caltech), for giving me the opportunity to do this interesting and challenging project. My client provided me needed data and resources for this project. I would also like to thank David Mispagel, Caltech Project Manager, for his great efforts in helping me to provide clean data in a useable and understandable format. David provided significant help to the project by giving me a lot of his valuable time for working with me in fixing and identifying data. I also thank Michael Uvaney, from Caltech, for his assistance in the preparation of needed data.

I would like to extend my gratitude to the City of Pasadena; in particular, Brian Sims, for providing aerial photos for the project area. I would also like to thank Shaun Farrell, from the Facilities Management Department at University of Virginia, for providing the attribute data for University of Virginia campus. 
I am also grateful to my dear friend Johnson Kosgei for his continuous consultation and support for the project and the program, as well. It was my honor and pleasure to have Mr. Kosgei as my roommate and classmate.

Last but not least, I would like to express my sincere appreciation to my friends in Cohort 14. This is the best cohort ever. We helped and treated each other as members of one family: thank you Alyssa, Bernard, David, Dorothy, Geraldo, Jennifer, Johnson, Joyce, Laura, Michael, Renee, Sean, and Tia for your inspiration, encouragement, and support. I would also like to extend my gratitude to Cohort 14 supporters Gabriel, Jasper, Matthew, Olga, and Rick. From other cohorts, I would like to acknowledge Ibrahima Almamy from Cohort 12 and Irene Muthuka from Cohort 15 for their assistance and support. 


\section{Abstract \\ Implementing GIS for Facilities Management at the California Institute of Technology}

by

Anas Bingaith

Most of the time managing facilities involve storing, querying, and updating spatial data. GIS is a perfect tool for building a database. The Facilities Department at the California Institute of Technology (Caltech) needed a method for more effective and efficient management of their facilities. Their map was not georeferenced nor interactive and did not have sufficient metadata. Their most current CAD files lacked accuracy. For any project, the Facilities Department faced many problems, including time spent searching for information, organizing data, difficulties finding accurate location of facilities, duplication of work, and difficulties in adding and updating data. Therefore, the project objective was to move CAD files to GIS. This involved georeferencing CAD files, converting CAD data to geodatabase feature classes, geodatabase design, and base map creation and design. These tasks were useful in assigning geographic coordinates to spatial data, providing better management of data, base map creation and design, and accuracy enhancement of CAD features. This project provided a basic understanding of how to work with CAD files in GIS, and outlined procedures and methodologies for geodatabase design and creating a base map for the facilities from CAD data. 



\section{Table of Contents}

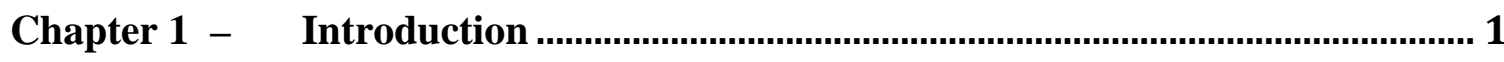

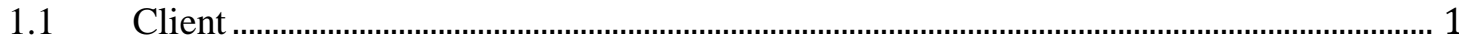

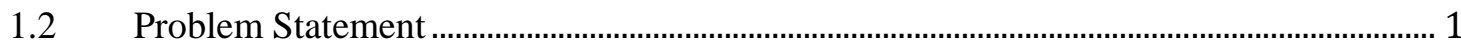

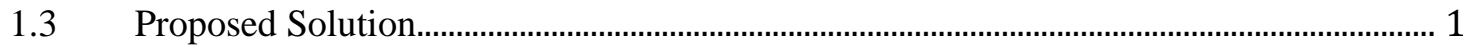

1.3.1 Goals and Objectives.................................................................................................... 2

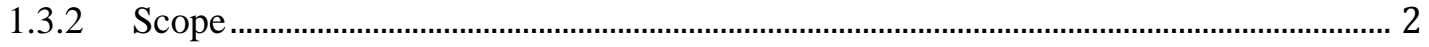

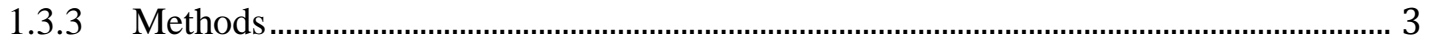

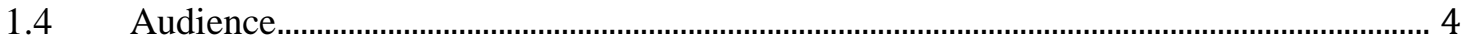

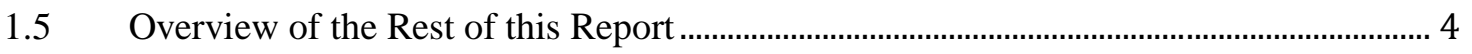

Chapter 2 - Background and Literature Review ……............................................... 5

$2.1 \quad$ Relationship between CAD and GIS........................................................................... 5

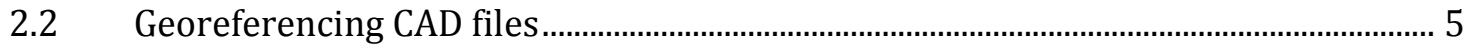

2.3 Examples of GIS Uses for Facilities Management at a University Campus ................. 6

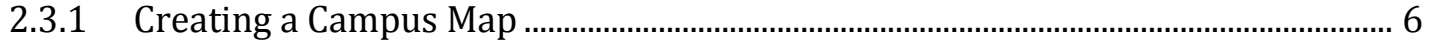

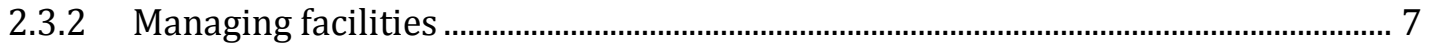

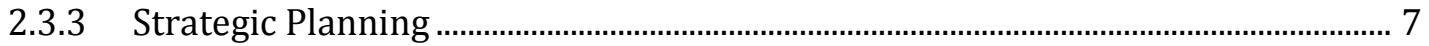

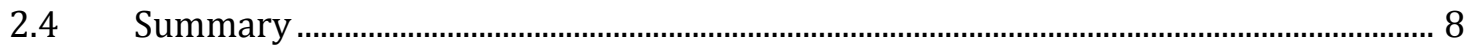

Chapter 3 - Systems Analysis and Design ………................................................ 9

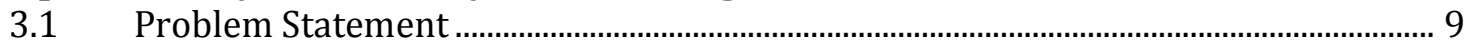

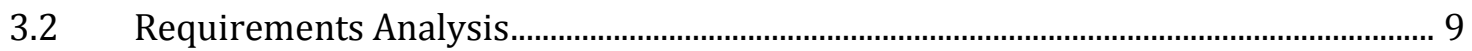

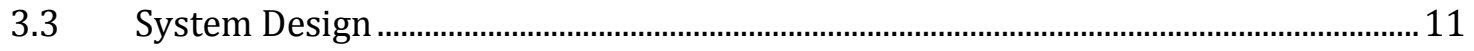

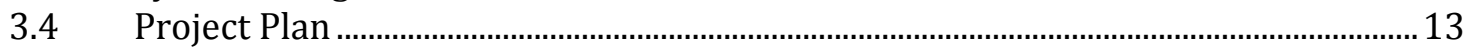

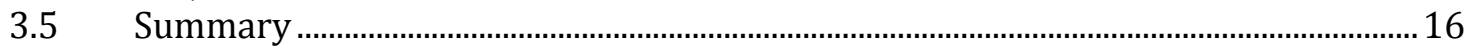

Chapter 4 - Database Design ...........................................................................17

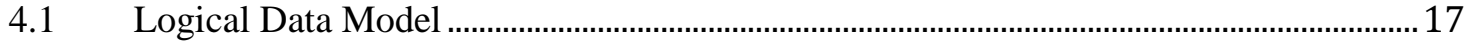

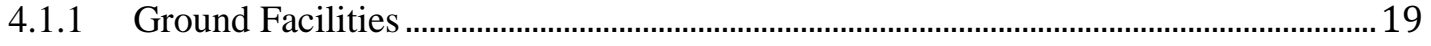

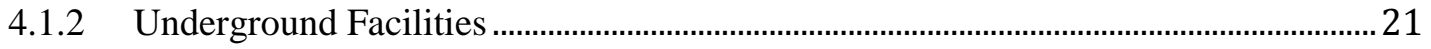

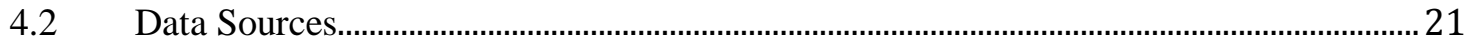

4.3 Data Collection Methods....................................................................................................22

$4.4 \quad$ Data Scrubbing and Loading .................................................................................................22

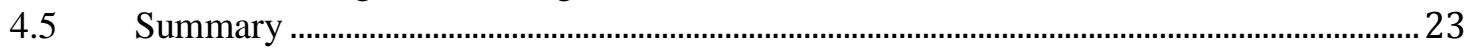

Chapter 5 - Implementation .............................................................................25

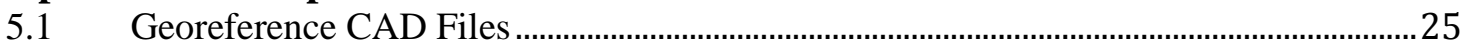

5.1.1 Assign Spatial Reference for CAD Files ..........................................................................2

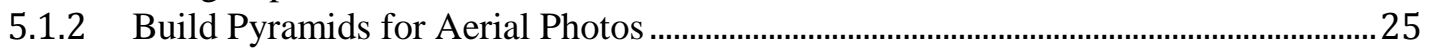

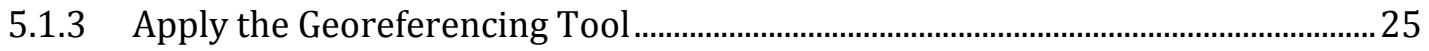

5.1.4 Test the Accuracy for Aerial Photos..................................................................................26

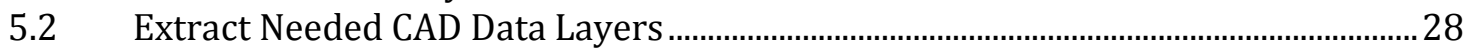

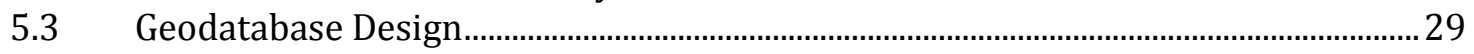

5.3.1 Convert CAD Data to Geodatabase Feature Classes .......................................................2

5.3.2 Organize Feature Classes and Raster Data Sets..............................................................30

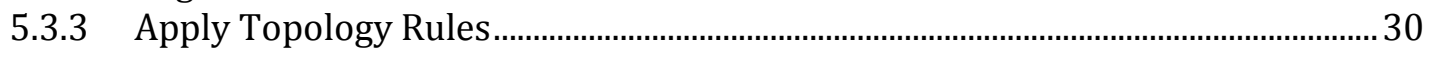

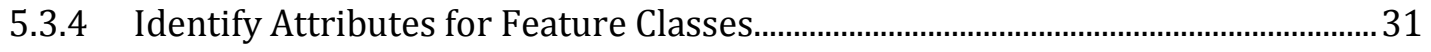

5.3.5 Create Subtypes and Domains............................................................................................... 31 


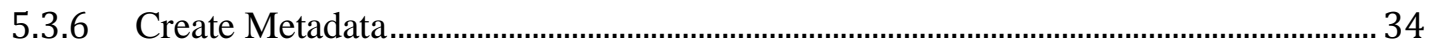

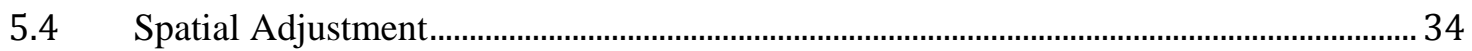

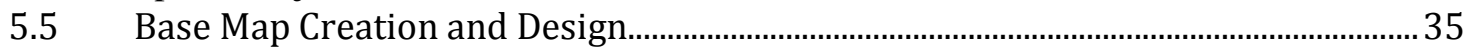

5.6 Convert Feature Classes to CAD Data............................................................................. 38

Chapter 6 - Results and Analysis ........................................................................39

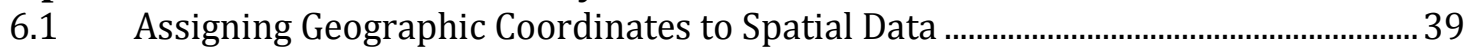

6.2 Providing Better Management of Data ................................................................................ 39

6.3 Base Map Creation and Design....................................................................................... 40

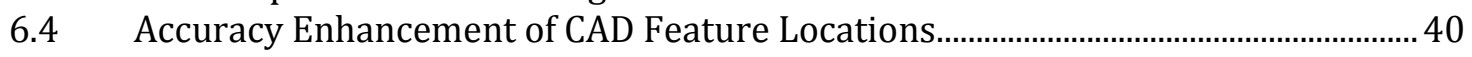

Chapter 7 - Conclusions and Future Work ……......................................................41

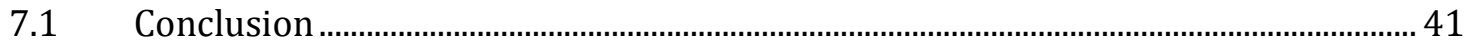

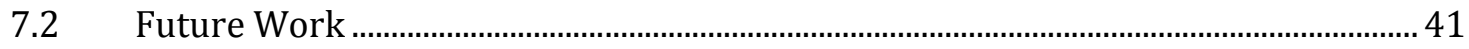

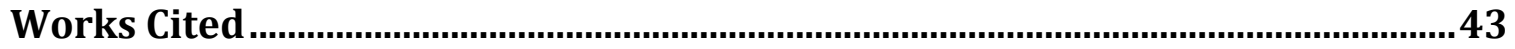

Appendix A. Schema Report ..................................................................................45

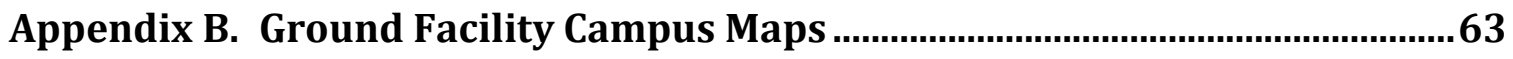

Appendix C. Underground Facility Campus Maps ............................................... 


\section{Table of Figures}

Figure 1.1: Project Methodology ............................................................................ 3

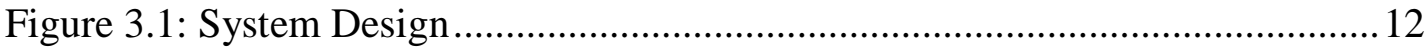

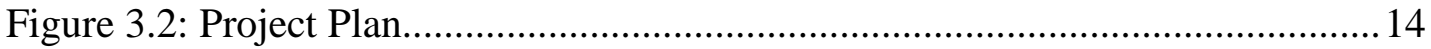

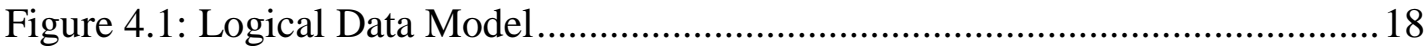

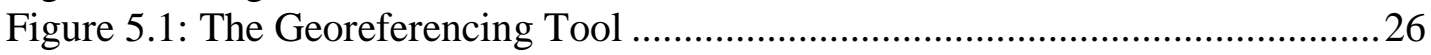

Figure 5.2: Location accuracy after georeferencing CAD features .........................27

Figure 5.3: Extract needed CAD data layers .....................................................28

Figure 5.4: Convert Coverage Annotation tool.....................................................29

Figure 5.5: Feature to Polygon tool converts only enclosed boundary objects ..........30

Figure 5.6: Convert features to polygons after applying topology rules .................... 31

Figure 5.7: Creating a subtype for manhole feature class...................................... 32

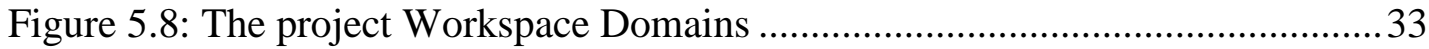

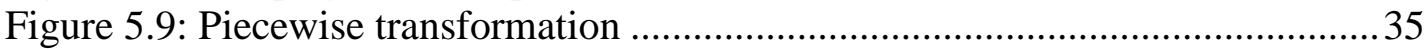

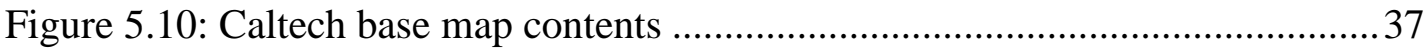





\section{List of Tables}

Table 1. Non-Functional Requirements ............................................................. 11 



\section{List of Acronyms and Definitions}

$\begin{array}{ll}\text { CAD } & \text { Computer Aided Design } \\ \text { Caltech } & \text { California Institute of Technology } \\ \text { ESRI } & \text { Environmental Systems Research Institute } \\ \text { GIS } & \text { Geographic Information Systems } \\ \text { DEM } & \text { Digital Elevation Model } \\ \text { BLOB } & \text { Binary Larger Object } \\ \text { GUID } & \text { Global Identifier } \\ \text { TIN } & \text { Triangulated Irregular Network } \\ \text { IDW } & \text { Inverse Distance Weighting } \\ \text { 3-D } & \text { Three Dimensional }\end{array}$





\section{Chapter 1 - Introduction}

Facilities management departments in most universities are responsible for a wide range of services, such as construction, operation and maintenance, utilities generation, and distribution. They are responsible for many important tasks regarding facilities including creating maps, locating underground utilities, spatial analysis and data querying, editing and updating data, and emergency response. Implementing a geographic information system (GIS) enables facilities departments to undertake these tasks and provide more effective and efficient management of facilities.

\subsection{Client}

Kenneth Hargreaves is the Senior Director of Design and Construction in the Facilities Department at the California Institute of Technology (Caltech). All capital improvement projects at Caltech, such as master planning and space management, are in his purview. The Design and Construction Division has 21 staff members who are mostly architects, engineers, and project managers to execute these projects. A primary function of the office is to serve as a communication and organizational hub on the campus for all fields of construction, renovation, and modernization. Mr. Hargreaves tasked Mr. David Mispagel, a Caltech Project Manager, to provide and identify needed data regarding the project.

\subsection{Problem Statement}

The current map of Caltech is not georeferenced. It is also not interactive nor does it have sufficient metadata. Due to these database limitations, the Facilities Department faced many problems while implementing projects which resulted in extra time spent on organizing and searching for relevant data. For example, to get information about a building such as address, name, and number, or any data about it, one has to search either computer aided design (CAD) drawing files or hard copy maps, and in some cases, both. Once a feature was identified, one had to further search for more information stored in related documents. These documents must be collected from different locations, and they are stored in different formats, such as Excel, Word, and PDF. Moreover, users in the Facilities Department found it difficult to relate disparate spatial data to the map because most data did not have geographic coordinate systems. Furthermore, attributes do not associate with maps in CAD. Therefore, queries and spatial analysis are impossible. Other problems include difficulties finding the accurate locations of underground facilities, duplication of work, and difficulties in editing and updating data. In addition, the Facilities Department required a related solution for master planning, space management, management, utility mapping, maintenance, and emergency response.

\subsection{Proposed Solution}

The approach to solving these problems with facilities management at Caltech was to convert CAD files to GIS. Implementing GIS for the Facilities Department provides a 
common coordinate system for spatial data, better management and organizing of various data sets, and an interactive base map.

The project assigned a coordinate system to all layers in the map to locate them in a correct place with their correct relative locations. Therefore, this simplifies finding accurate locations of facilities, especially underground facilities. For example, one can display underground facilities layers in registration with an aerial image layer, and then make precise measurements.

In a GIS, databases associate with maps. Thus, analyses and queries can be applied to spatial data. For this project, a file geodatabase was created to hold all data sets. A geodatabase has the unique advantage of storing database tables, map information, and metadata in a central repository. Unlike GIS, in CAD related data sets may require to be stored in different formats or at different locations. Thus, GIS makes it easy for users to find information immediately, as well as to organize, edit, and update data. Furthermore, data entry can be automated and validated in GIS.

A base map that contains all layers of interest was compiled. Users can add or display any layers of interest from many different sources of spatial data. All map elements can be easily controlled using GIS including labels, symbols, and scale of display.

\subsubsection{Goals and Objectives}

The main goal of this project is to support the Facilities Management Department at Caltech to manage facilities in an effective and efficient way by implementing GIS. The expected outcome is a file geodatabase, base map, and better accuracy of CAD feature locations to support managing, planning, and decision making.

\subsubsection{Scope}

The scope of this project and the client's expectation were to georeference CAD files, design geodatabase, and create a base map for the Caltech campus. Caltech has 125 buildings spread over a 124 acre campus. Three important tasks were applied to this project: moving CAD files to GIS, designing the geodatabase, and creating a base map.

At the beginning of this project, there were more than 500 CAD files that were provided by the client. However, not all these were needed for the project. Moreover, many of these data had layers showing only underground facilities of which these underground facilities layers cannot be easily georeferenced to the aerial photos. Furthermore, some CAD features that could be georeferenced were found to be not accurate in their locations. There was shift about three to ten feet in different locations. The only solution for that was to use the spatial adjustment tool and apply the Rubbersheet method. However, the client had different CAD files which had very accurate locations of their features, but there were few of them. Thus, later on, in middle of working in the project, the client decided to provide these accurate few CAD files to be used for the project with the old files. Thus, the scope was changed to include some of the CAD files and to document the procedures so that the client may apply them to other CAD files.

The hardware component of this solution consisted of one computer. The software component was ArcGIS 9.3 desktop tools and functionality includes ArcInfo and 
ArcCatalog. The data components were 13 CAD files sets and 9 aerial photos that cover the study area.

This project required four main GIS skills; working with CAD files in GIS, geodatabase design, spatial adjustment, and base map creation and design.

\subsubsection{Methods}

The techniques and methods that were used to complete the project are illustrated (Figure 1.1) and described below.

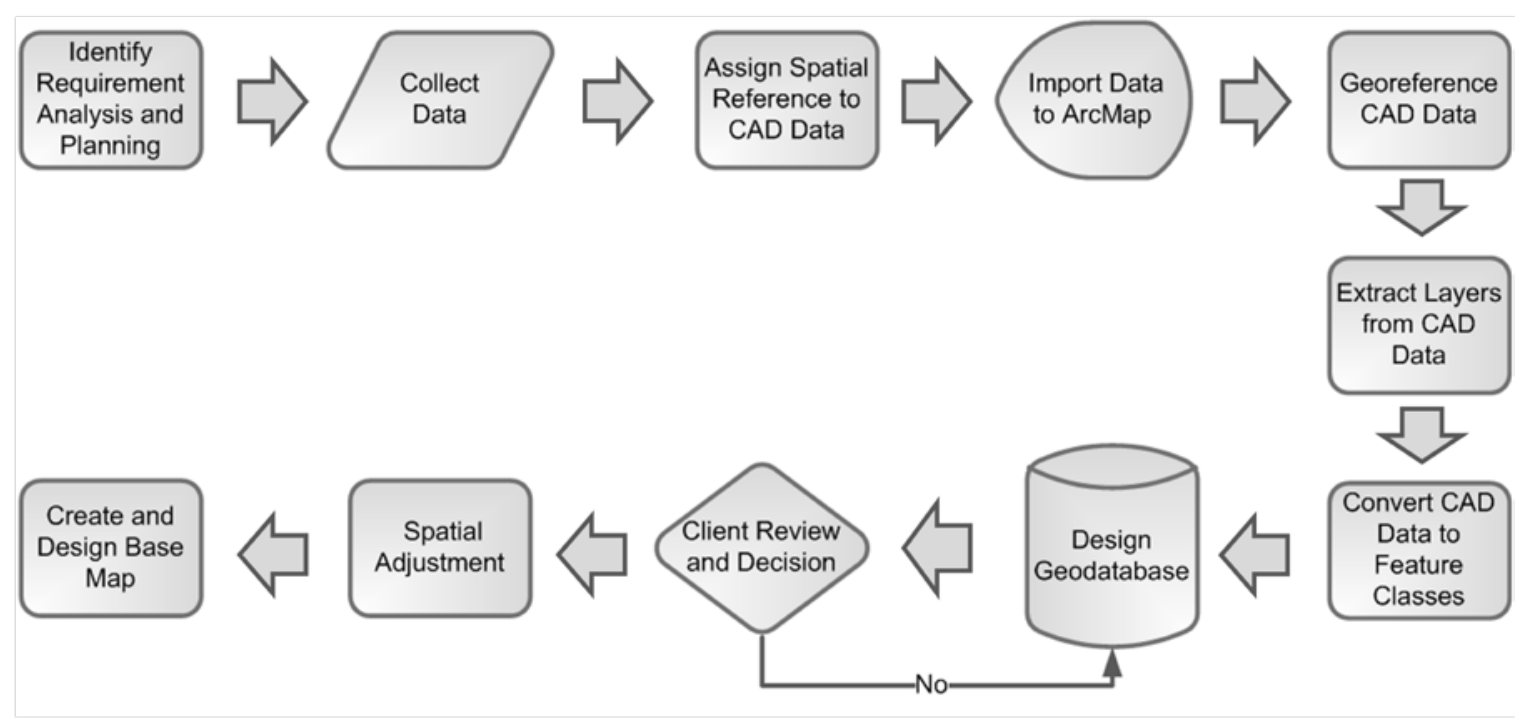

Figure 1.1: Project Methodology

1. Identify requirement analysis and planning: In this task, the problem and solution were identified, scope and goals were developed, and a project plan was created.

2. Collect data: CAD files and aerial photos were collected.

3. Assign spatial reference to CAD files: CAD files of interest were selected, given clear names, and assigned spatial reference.

4. Import data to ArcMap: CAD files and aerial photos were imported to ArcGIS.

5. Georeference CAD files: CAD files were given geographic coordinates and projection to define their locations on earth.

6. Extract layers from a CAD data: A CAD data contained many layers. For example, one CAD data had separate layers for buildings, grounds, parking, and fire hydrants. It was necessary to create separate CAD data for each of these layers; for example, one CAD data for only buildings, one for grounds, and so on.

7. Convert CAD data to feature classes: A file geodatabase was created. Then CAD data were converted to feature classes in the file geodatabase.

8. Design the geodatabase: This task involved in identifying attributes, organizing feature classes, creating topology, and compiling metadata.

9. Client review and decision.

10. Adjust spatial data: This task was applied to establish more accurate location estimates using the Rubbersheet method. 
11. Create and design a base map: This task involved assigning symbols and scales to features, and creating and designing a new base map.

\subsection{Audience}

This project report is intended to be helpful to both GIS users and non-GIS users. For non-GIS users, such as the client for this project, it gives them the sense of understanding how CAD files work in GIS, and also the relationship between GIS and CAD. For GIS users, it gives them understanding and basic ideas of how to move CAD files into GIS. It focuses on converting and georeferencing CAD files into a GIS, and working with it within a GIS environment. Moreover, it focuses on designing a geodatabase from CAD files and creating a base map. It also allows users to improve the positional accuracy of the CAD features, and discern the potential source of error. This is attained by overlaying CAD data with data from known reliable sources. In general, this report is helpful for any person who wants to work with CAD files in GIS, and design a geodatabase or base map.

\subsection{Overview of the Rest of this Report}

In general, the project report explains four main tasks: move CAD files into GIS, design the geodatabase, adjust spatial data, and create and design a base map. The project report divided to seven chapters.

Chapter two is about background and literature review. It provides research about the relationship between CAD and GIS, georeferencing CAD files, and examples of GIS uses for facilities management on university campuses.

Chapter three, which is about system analysis and design, explains the client's problem, and discusses the requirements analysis for functional and non-functional requirements. In addition, it presents the system design and project plan.

Database design, Chapter four, explains how this project was done. It describes the design of a geodatabase, and provides the reasons for designing it this way. It illustrates the logical data model. This model discusses the feature data sets and their relationships, and describes the feature classes and tables. It investigates important metadata, and where data came from. Finally, it describes the data collection methodology, and the methods for cleaning the data before importing it to the geodatabase.

Project implementations were explained in Chapter five. It describes in details the implementation of GIS for georeferencing CAD files, geodatabase design, accuracy enhancement of CAD feature locations, and base map creation and design.

Chapter six presents analysis, and the results that were important for the client. It also discusses problems and the reasons behind them.

The last chapter is Chapter seven, which is about conclusion and future work. It summarizes the goals, methods, and the results of the project. Moreover, it suggests some potential extensions to the project. 


\section{Chapter 2 - Background and Literature Review}

Safety, high quality service, beauty, and exceptional facilities are all important to the quality of a university campus. Thus, a facilities management department is an essential part of every university, and oversees tasks such as operating and maintaining campus facilities, grounds and infrastructure, and providing an attractive, clean, accessible, safe and well planned educational environment for campus activities. Moreover, it supports scientific and research projects. GIS has all the functionality necessary to make it the perfect tool to manage these responsibilities.

Many facilities departments are using computer aided design (CAD) software for drawings and maps. Recently, however, many are adopting GIS to work with their CAD files. Thus, it is important for a facilities manager who wants to implement GIS to understand the relationship between GIS and CAD, and how CAD files can be used with a GIS. In addition, it is important to know what GIS can do to support facilities departments.

\subsection{Relationship between CAD and GIS}

A GIS is a computer-based system to aid in the collection, maintenance, storage, analysis, and distribution of spatial data and information (Bolsted, 2008). However, computer aided design (CAD) is the use of computer technology to create precision drawing or technical illustrations in two or three dimensions. It is used primarily by architects, engineers, and artists (Lewis \& Nell, 2007). CAD and GIS are different in many areas such as modeling, objects, topology, and data management.

According to Kathryn Clifton, a GIS Coordinator in the City of Salisbury, at the 1998 Autodesk Design World, Bill Wittreich of Wittreich \& Associates, gave a presentation titled "Beyond CAD into GIS." What follows is paraphrased from a portion of his talk: CAD is excellent for drawing while GIS is excellent for spatial database operations. Objects in a CAD environment, such as points, lines, and polygons, do not have relationships between them, and they do not relate to each other. Features in CAD might touch or overlap. Thus, it is impossible to create networks in CAD systems due to it has limitations in handing connectivity. However, objects in GIS have a coordinate system that locates them relative to fixed places on earth. Another difference between CAD and GIS is that GIS has topology while CAD does not. Therefore, it is possible to create networks in GIS having enclosed areas. In GIS, objects have relationships between them. A user can create node topology, network topology, and polygon topology. This allows for spatial analysis and modeling of direction and resistance. Furthermore, a CAD system is usually memory-based whereas GIS uses disk-based storage. Thus, GIS can model more extensive areas than CAD systems. GIS also separates object storage from object display while CAD does not separate objects (Kathryn, 2002).

\subsection{Georeferencing CAD files}

Georeferencing is a process to assign geographic coordinates to raster or vector data, and define their existence in the physical space on Earth. This process links unknown coordinates of raster or vector data to a known coordinate system. A CAD file is usually 
quite accurate relative to a local coordinate system. However, GIS has the unique power of relating CAD drawings to a map with data from many sources in a global coordinate system (SBL Geomatics Group, 2009).

According to ArcGIS Desktop Help 9.3 (ESRI, 2008), spatial data must have a geographic or projection coordinate system in order to use all the functionality of a GIS. Raw CAD files often have unknown projections and geographic coordinate systems. Therefore, the first step to work with CAD data in GIS is to georeference the CAD data. CAD data can be georeferenced by applying three steps. The first step is defining a coordinate system for CAD files by using ArcCatalog, which provides the ability to choose predefined coordinate systems, import coordinate systems, or define a new coordinate system. When CAD files are defined, the coordinate system must be stored as projection file (.prj) in the same CAD files folder to save the spatial reference for the CAD files. The second step is transforming CAD files by using the Georeferencing tool in ArcMap. This tool applies only two control points for accurate locations of CAD features because CAD drawings only require similarity transformation that supports rotation, shifting, and scaling. This transformation preserves uniform scaling, and applies to the target layer that is specified in the Georeferencing tool. To have a control point, two set points are needed. The first one must be in the source data needed to transform its coordinate system, and the second one must be in the destination data needed to have the same its coordinate system. The third step is updating georeferencing. This step should be applied when the transformation of CAD files is complete. It defines the transformation and the coordinate system for the CAD files by creating a text file called a world file (.wld). This file should be saved in the same CAD file folder and contains the coordinates for the two control points (ESRI, 2008).

Some CAD data, however, are not accurate due to geometric distortion, and need more than two control points. The Rubbersheet method from the Spatial Adjustment tool is the perfect way to perform geometric adjustment. However, in order to work with this tool, CAD data must be converted to a feature class. The Rubbersheet method is used to adjust features with more accurate information. It uses displacement links to adjust features that move dependently on nearby displacement links. The feature that near from displacement links, is the more it will move (ESRI, 2008).

\subsection{Examples of GIS Uses for Facilities Management at a University Campus}

Facilities departments can use GIS for many important applications that support efficient management of facilities. GIS can be used to create a campus map, manage facilities, and for strategic planning.

\subsubsection{Creating a Campus Map}

GIS can be used to create a base map of a campus. Some data layers available from public sources, such as 3-D topographical surfaces, aerial photos, parcel boundaries, roads and paved areas, watercourses, wetlands, floodplains, soils boundaries, building footprints, utilities, landscaping, photos set of all exterior lights, photo set of all buildings, CAD drawings of new and recent building project, as-built drawing, old maps, and blueprints. GIS can use these layers and their information as campus virtual tours so 
that prospective students can explore the campus. It will help them easily and quickly identify campus features and their attributes, including their history and photographs of them (Weiss, 2009). For example, at Troy University, a 3-D campus map was created. ArcGIS was used to generate the 3-D model of campus. The ArcScene application was used to show the 3-D model. The 3-D model was generated using a computed digital elevation model (DEM). The DEM provides the height information in the 3-D model (Ramroop, 2006).

\subsubsection{Managing facilities}

GIS is an excellent tool to provide efficient and effective management of facilities whether they are ground or underground facilities. Most challenges that a facilities manager faces include dealing with managing underground facilities. The common issues with underground facilities include finding the exact location for underground facilities, network analysis, and maintenance. With a GIS, one can easily identify exact location of underground facilities. This can be identified by overlaying an aerial photo or any other ground feature with underground facilities, and then perform measurements. Also, GIS can provide network-based spatial analysis for utilities including flow direction and topology creation. Moreover, GIS can be used to track work orders such as landscape and utilities maintenance. For example, a list of maintenance tasks showing the description and location in the work order. In landscape maintenance, GIS can be used to display locations of different kinds of trees, flowers, and other plants on a map and the schedule for regular requirements of watering, pruning, weeding, and fertilizing. Thus, GIS can help in maintenance of landscape appearance and increases productivity of the facilities department staff (Weiss, 2009).

\subsubsection{Strategic Planning}

GIS can be used to create useful tools and maps to be used in strategic planning, such as accessibility planning, pedestrian planning, emergency planning and response, and facility space planning.

GIS can display and illustrate handicap accessible buildings, routes, and parking. Disabled visitors or students can have maps showing primary and auxiliary handicap accessible routes, location of handicap parking spaces, buildings that are handicap accessible, location of sidewalk curb cuts and handicap ramps, and handicap accessible entrances (Weiss, 2009). Ferries State University (FSU) has implemented GIS to provide the best service for people who have disabilities, and make sure that FSU meets the Americans with Disabilities Act (ADA) and the university standards. The GIS project was mapped out the handicap accessibility facilities (parking spaces and their accessibilities to the surrounding buildings). In addition, it was used aerial photographs and various mapping techniques (e.g.; handheld GPS) to map and identify accessibility routes and facilities (London, 2006).

GIS is a perfect tool for pedestrian and transportation planning. For example, one can create a map with GIS showing path locations on campus such as pedestrian paths, bicycle routes, and bus stops and routes. It can include parking areas for cars, bicycles, and motorcycles as well as display visitors, and student parking spaces in different colors (Weiss, 2009). "Parking, Transit and Traffic services (PTTS) at Texas A\&M University 
is using the campus GIS for mapping and managing bus routes, striping work on streets and parking lots, keeping track of signs, and managing parking lot allocations. PTTS is planning, with the Police Department, to implement a dispatching system for bus, police and 911 emergency uses” (Bassham, 1994).

GIS is also important tool for emergency planning and response. For example, it can show fire hydrants and display their locations. In the event of a fire or earthquake, a campus planner can use GIS to tell if the area is at risk or not. GIS can locate and show evacuation routes and the best areas to evacuate. In addition, it helps a campus planner under any emergency to quickly locate water and gas shut-off valves (Weiss, 2009).

GIS can also show detailed floor plans of campus buildings. It can be used to manage classroom space, identify all features in the classroom, and for emergency response (Weiss, 2009).

\subsection{Summary}

GIS is a powerful tool for facilities management. Most facilities management departments have CAD data. Thus, implementing GIS for facilities management involves working with CAD files. The first step for doing so is to georeference CAD files, design a geodatabase, and create a base map. Once the base map has been created, all facility information can be compiled and added to the system. Then, many GIS applications can apply to help Facilities Management departments to manage facilities in an effective and efficient way. Therefore, this project intends to create the fundamental base of implementing GIS to support efficient management of facilities at the California Institute of Technology (Caltech). This fundamental base provides a facilities manager the knowledge of how to work with CAD files in GIS, how to design a geodatabase, and how to create a base map. 


\section{Chapter 3 - Systems Analysis and Design}

This chapter identifies the key elements before starting the project implementation. These elements are: understanding the problem, requirements analysis, project design and components, and a project plan.

\subsection{Problem Statement}

Identifying and understanding the client's problems are the first important step to successfully implementing the solution. The client had many problems regarding managing facilities. First, the client had difficulties finding exact locations for underground facilities. CAD drawings used to locate the underground facilities were not accurate because they were created from a photo that was not an orthophoto. Also, surveying measurements were not used in the initial creation of CAD drawings. Thus, measurements from resulting CAD features did not yield the accurate locations for facilities. Second, the data needed to be more organized and categorized to be easy to manage. Data were stored in different formats. For example, some drawings were stored in digital formats, such as AutoCAD files, jpeg, or tiff, and others existed only as hard copy. Information regarding spatial data was also stored in different formats, including hard copy and digital formats such as Excel, PDF, and Word documents. Moreover, most of the spatial data lacked sufficient metadata. Thus, the client spent considerable time editing, updating, organizing, and searching for information. Any change in the spatial data required a preliminary search for all relevant data format. Therefore, the client needed to manage the facilities data more efficiently and effectively. The map of Caltech was also not georeferenced. The Facilities Department requires a related solution for master planning, space management, utility mapping, maintenance, and emergency response. However, data for these sources could not be combined and used because of the lack of a reference system. Also, users could not produce maps that link two or more data sets together.

\subsection{Requirements Analysis}

The GIS system should meet the following mandatory requirements:

1. Users should able to relate CAD data and feature classes to many sources of spatial data in a global coordinate system.

2. The system should store and organize data sets in a file geodatabase.

3. Capability to handle enclosed polygons.

4. Users should edit, query, and analyze spatial data in efficient way.

5. Capability for storage and retrieval of metadata for data sets.

6. The system should display base map layers with its design.

7. Accommodate enhance accuracy of CAD features.

8. Users should be able to apply all GIS functionality including topology and spatial connectivity.

CAD files were provided with unspecified projections and geographic coordinate

systems, so it was difficult to link them with spatial data. Thus, the system should provide 
georeferenced CAD files that have defined feature coordinates, so users can relate CAD data with other data from varying sources based on a global coordinate system.

The system should store and organize data sets in one container called a file geodatabase. Thus, data sets can be easily updated and edited, and easily organized and categorized. Feature classes can be categorized into different feature data sets. A feature dataset can include many feature classes, such as annotation, fittings, ground facilities with their topology and geometric network, and underground facilities with their topology and geometric network. In addition, a collection of relationships between objects can be created in tables or feature classes.

In order for users to calculate an area or apply polygon topology, polygon features must an enclosed boundary. However, the client's CAD features did not contain true polygons. Therefore, the system should handle enclosed polygons.

The system should provide identified attributes for feature classes so that users could visualize, query, and analyze spatial data. CAD properties such as line color, width, and thickness are treated in ArcGIS as attributes. These attributes are not useful in GIS, and therefore the system should exclude them. Subtypes and domains provide an efficient way for editing and use less storage space in the geodatabase, so the system should contains subtypes and domains.

Metadata files are valuable documents that store important information about data. The system should provide metadata that includes properties and descriptive information about each dataset in the project.

The system should have the capacity to overlay base map layers, including aerial photos, ground facilities, and underground facilities. This would help to determine the exact locations of underground facilities by georeferencing them to aerial photos or features of ground facilities. It should also show the base map design, including scales, labels, and symbols. Users can also use this system to produce maps from different data sets efficiently.

Most of CAD features were drawn from ungeoreferenced photos; furthermore, accurate surveying measurements were not referred to when the CAD features were first created. Thus, it lost most of its geometric accuracy. Moreover, in order to use all GIS functionalities, CAD files must have a reference system. Therefore, the implemented solution should provide georeferenced data that is sourced from reliable sources so as to ensure better accuracy of CAD feature locations.

The system should allow users to apply all GIS functionality. Users could use the GIS system to apply spatial analysis, such as measuring, selecting, identifying, querying, visualizing, and filtering data. For example, measurement tools can be used to get the exact locations for underground facilities from aerial photos or ground facilities. Also, different kinds of selections are available in the system. Any feature on the map can be selected from the attribute table, and it is possible to select it based on its location relative to other features. It is possible also to select features by drawing graphics or simply using the select feature tool. The system provides the identify tool. When the user clicks or drags a box over a feature, the attributes will be listed in the identify Dialog box. Any features or attributes of interest can be queried. For example, the user can query and visualize pipes that were installed more than ten years ago in order to replace them. These pipes can in turn be filtered to show only copper. Users can then print maps that show the 
exact locations for these old copper pipes with their attribute tables in an effective and efficient way.

The non functional requirements are as follows (Table 3.2). First, the system requires using ArcMap and ArcCatalog from ArcGIS software. ArcCatalog is used to manage and organize various data sets, while ArcMap is used to display, explore, analyze, assign symbols, print, publish, and create maps. Second, the delivery of the project should be as follows: georeferenced CAD files with better accuracy of their feature locations, base map, file goedatabase, and project report. Some CAD drawings that users in the Facility Department worked on using AutoCAD software had bad accuracy. So it was necessary for providing better accuracy of CAD feature locations. The base map was delivered in ArcMap file (.mxd), so the client had a map containing features with the appropriate symbols and scales. Also, ArcMap file makes it easy and quick to publish, display, and print maps. File geodatabase is the most important product for the project. It contains all data sets, such as tables, feature classes, and raster data sets. The last part of delivery was the project report which contains information and general details about the project. Finally, the project might be presented to the client. It depends on the client time availability.

\section{Table 1. Non-Functional Requirements}

\begin{tabular}{|c|l|c|}
\hline & \multicolumn{1}{|c|}{ Requirement } & Priority \\
\hline 1 & Use ArcGIS software & Mandatory \\
\hline 2 & $\begin{array}{l}\text { Deliver file geodatabase, base map, georeferenced CAD files } \\
\text { with better accuracy of their feature locations, and project } \\
\text { report }\end{array}$ & Mandatory \\
\hline 3 & Present the project to the client & Desirable \\
\hline 4 & Provide a project poster to the client & Desirable \\
\hline
\end{tabular}

\subsection{System Design}

The system is categorized into three major components (Figure 3.1): input, process, and output. Input refers to data input. Process refers to software components and tools that were used to execute the project. Output refers to the final products of the project. 


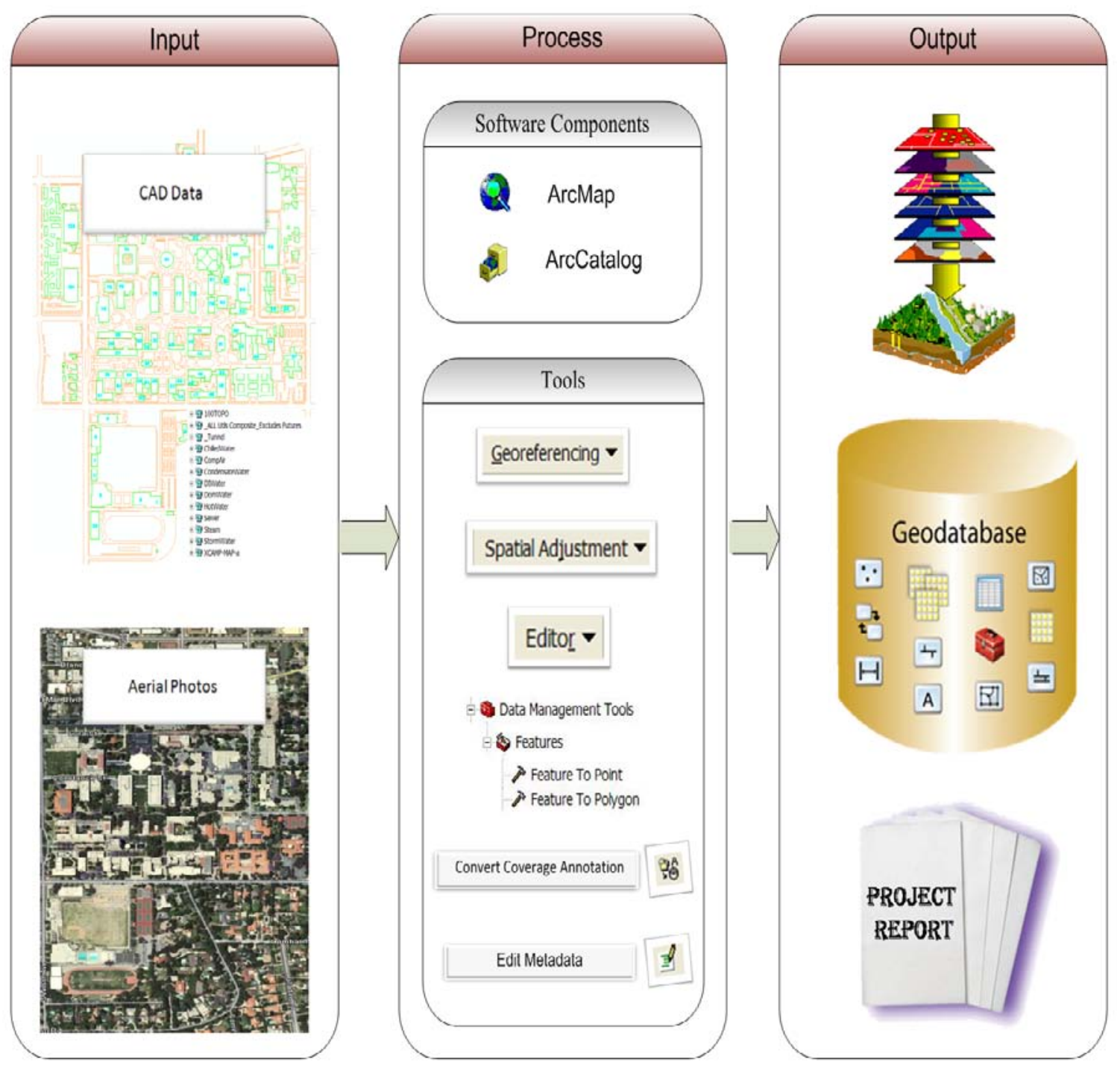

Figure 3.1: System Design

Input components are aerial photos and CAD files. The City of Pasadena provided nine aerial photos for the project area, taken in the winter of 2006. Each photo covers 0.25 square mile. Aerial photos are important for georeferencing CAD files, and can be used for spatial analysis with other data sets. The second component of input was CAD files. The first time the client provided CAD files; there were approximately $580 \mathrm{CAD}$ files, created by many sources and with different accuracy. Also, most the names for these CAD files were written as codes that needed to be searched from an index book to understand what each was for. An appointment was arranged with the client to discuss these issues. The client subsequently decided to provide only CAD files of interest with clear names. This minimized the data to cover thirteen files. These CAD files included ground facilities and underground facilities. Ground facilities included buildings, streets, tree cover, palms, parking lots, fire hydrants, pool areas, pole lights, and manholes. Underground facilities included compressed air pipes, storm water pipes, steam pipes, 
sewer pipes, hot water pipes, chilled water pipes, tunnel, condensate water pipes, demonized water pipes, and domestic water pipes.

The process component was categorized for software components and tools components. Software components included ArcMap and ArcCatalog. Tools components included the following: First, the Georeferencing tool, with three tasks: fit to display, auto adjust, and update georeferencing. The second tool, Spatial Adjustment, had four tasks:

Set Adjust Data, the Rubbersheet in the Adjustment Method, Adjust, and a New Displacement Link icon. Third, the Editor tool was used to enable using the Spatial Adjustment tool. The Sketch Tool icon in the Editor tool was also used for digitizing. From the Data Management Tools in ArcToolbox, the Feature To Point tool and Feature To Polygon tool were used for converting features. However, for converting annotations, Convert Coverage Annotation icon was used. Finally, Edit metadata icon was used to create the metadata.

The final products that represented output in the system design included: a base map in ArcMap document, a file geodatabase including all collection of data set, and a project report.

\subsection{Project Plan}

The project plan (Figure 3.2) was created in early December, 2008 and represents task duration rather than the actual work-time. The project plan contains thirteen tasks that were used for guidance to successfully deliver the project. 


\begin{tabular}{|c|c|c|c|c|c|c|c|}
\hline \multirow{2}{*}{ ID } & \multirow{2}{*}{ Task Name } & \multirow{2}{*}{ Start } & \multirow{2}{*}{ Finish } & \multirow{2}{*}{ Duration } & Q408 & Q1 09 & Q209 Q309 \\
\hline & & & & & \multicolumn{3}{|c|}{ 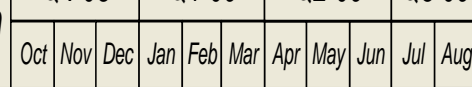 } \\
\hline 1 & Project Planning and Requirements Analysis & 9/29/2008 & 12/15/2008 & $78 d$ & & & \\
\hline 2 & Define Problem and Solution & 9/29/2008 & 10/23/2008 & $25 d$ & 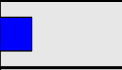 & & \\
\hline 3 & Develop Scope and goals & 10/23/2008 & $11 / 5 / 2008$ & $14 d$ & $\bar{\square}$ & & \\
\hline 4 & Create Project Plan & $11 / 5 / 2008$ & 12/15/2008 & 41d & $\square$ & & \\
\hline 5 & Data Collection & $11 / 28 / 2008$ & $1 / 5 / 2009$ & $39 d$ & $\bar{E}$ & & \\
\hline 6 . & Training and Research & $1 / 5 / 2009$ & $3 / 21 / 2009$ & $76 d$ & & & \\
\hline 71 & Data preprocessing & $3 / 21 / 2009$ & $4 / 13 / 2009$ & $24 d$ & & & 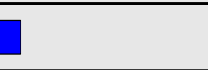 \\
\hline 81 & MIP Documentation & $4 / 13 / 2009$ & $8 / 10 / 2009$ & $120 d$ & & & \\
\hline 9 & Georeferencing & 4/14/2009 & $5 / 14 / 2009$ & $31 d$ & & & $\square$ \\
\hline 10 & CAD Data Conversion & $5 / 15 / 2009$ & $5 / 16 / 2009$ & $2 d$ & & & I \\
\hline 11 : & Spatial Adjustment & $5 / 17 / 2009$ & $6 / 1 / 2009$ & $16 d$ & & & $\square$ \\
\hline 12 & Geodatabase Design & $6 / 2 / 2009$ & $7 / 14 / 2009$ & 43d & & & 7 \\
\hline 13 & Identify Attributes & $6 / 2 / 2009$ & $6 / 20 / 2009$ & 19d & & & $\square$ \\
\hline 14 & Organize Feature Classes & $6 / 21 / 2009$ & $7 / 5 / 2009$ & $15 d$ & & & 口 \\
\hline 15 & Compile the Metadata & $7 / 6 / 2009$ & $7 / 14 / 2009$ & $9 d$ & & & 1 \\
\hline 16 & ESRI User Conference Preparation & $7 / 2 / 2009$ & $7 / 17 / 2009$ & $16 d$ & & & $\mathbf{T}$ \\
\hline 17 & Prepare the Presentation & $7 / 2 / 2009$ & $7 / 17 / 2009$ & $16 d$ & & & $\square$ \\
\hline 18 & Prepare the Poster & $7 / 2 / 2009$ & $7 / 17 / 2009$ & $16 d$ & & & $\square$ \\
\hline 19 & Base Map Creation and Design & $7 / 18 / 2009$ & $8 / 10 / 2009$ & $24 d$ & & & $\square$ \\
\hline 201 & MIP Defense & $8 / 11 / 2009$ & $8 / 11 / 2009$ & $1 d$ & & & I \\
\hline & Deliverable & $8 / 12 / 2009$ & $8 / 12 / 2009$ & $1 d$ & & & I \\
\hline
\end{tabular}

Figure 3.2: Project Plan

The first task was project planning and requirements analysis. It took two and a half month to complete and contained three subtasks, including defining the problem and solution, developing scope and goals, and project planning. It took around 72 hours of actual work time over 25 days to understand the client problem and identify the ideal solution. The next step was developing scope and goals. It took 14 days to determine how far this project could go, and what it could achieve. The last step of the first task was creating a project plan. It took 41 days to identify project tasks and predict the time necessary to complete each task. 
The second task was data collection. It was planned to receive all data needed within 39 days. CAD files arrived in sufficient time from the client. However, the aerial photos took 46 days to receive because I had to contact the city of Pasadena in order to request them, and then sign licensing agreements for releasing the data. Thus, this data was a week late.

Due to my limited experience of working with CAD files in GIS, training and research were an important consideration. The MS GIS program at University of Redlands arranged a training class for working with CAD files in GIS on the $20^{\text {th }}$ of March. The plan was to do some research before the training day to know more about CAD files and prepare for how to work with them in GIS.

The fourth task and first implementation task was data preprocessing. It was planned to complete within 24 days. However, due to the limited experience of project implementation and unexpected circumstances, the reality was different from the project plan for all project implementation tasks. For example, CAD files were expected to be clean and having accurate locations of their features, but they were not. This was discovered after spending time to check the accuracy of aerial photos and georeference the CAD files. Also, during data preprocessing, CAD files of interest were selected and given meaningful names, then assigned a spatial reference. The data preprocessing task took 55 days, until the middle of May.

The fifth task was the documentation. It was started on the $5^{\text {th }}$ of July, a month late than what was planned. It is expected to complete by May 2010 which is different than the plan due to personal circumstances.

The second task of implementation was georeferencing CAD files. It was planned to be finish by the middle of May, within 31 days. However, it was started by the middle of May, and finished by the end of May, within only 15 days.

The third implementation task and the seventh in the project plan was CAD data conversion. It was planned to finish it in two days. However, this task involved some complex issues. For example, for a building feature that converted from polyline in CAD to polygon feature in GIS, some features were not converted because they were a nonclose area. Fixing this problem required to two more tasks: creating topology and digitizing some features. Some CAD data were also impossible to extract some of their layers from ArcGIS. It was required to fix that in the AutoCAD environment and took one month to complete.

Having inaccurate locations of CAD features caused inaccurate feature classes. Thus, a spatial adjustment task was applied to provide better accuracy of feature classes. Also, the client needed to have better accuracy of CAD feature locations to work with it in AutoCAD. Therefore, feature classes were converted to CAD files. These two tasks took one week. The plan to complete this task was expected to complete by the first of June. However, it was completed by $22^{\text {nd }}$ of June.

The ninth task was geodatabase design. It included three subtasks: identify attributes, organize feature classes, and compile metadata. This task should have started immediately after data collection because attributes data take a long time to collect. Thus, attributes were identified without collecting their data. It took 18 days to complete it.

Environmental Systems Research Institute (ESRI) User Conference preparation was the tenth task in the project plan. It included two subtasks: presentation preparation and 
poster preparation. The task was started on the $2^{\text {nd }}$ of July as the expected plan, and was completed two days earlier than planned.

The last implementation task was base map creation and design. This task included designing the campus maps include symbols and labels for feature classes, and to specify the scale range at which each layer would be shown. Then it was saved in ArcMap document. This task was started on the $23^{\text {rd }}$ of July, and completed by the $10^{\text {th }}$ of August.

The last task in the project plan was the deliverable. Project products such as file geodatabase, ArcMap document, and georeferenced CAD files were delivered to the client on date. Project report, however, is expected to deliver by May 2010.

\subsection{Summary}

The client wanted to implement GIS to be a solution for many problems such as finding exact locations for underground facilities, organizing and managing data, and enhancing the accuracy of CAD feature locations.

The functional requirements are as follows: the system should provide georeferenced CAD data with better accuracy of their feature locations, display all layers in the base map, and provide effective management of data. Spatial analysis also can be applied, and different maps for analysis can be printed. In the non functional requirements, the system requires using ArcMap and ArcCatalog. The project should deliver georeferenced CAD files with better accuracy of their feature locations, base map, file goedatabase, and project report. Finally, the project might be presented to the client. It depends on the client time availability.

The system is categorized into three major components. First, input components, which included aerial photos and CAD files. Second, process components, was categorized for software components and tools components. Software components included ArcMap and ArcCatalog. Tools components included Georeferencing tool, Spatial Adjustment tool, the Editor tool, Feature To Point tool and Feature To Polygon tool, Convert Coverage Annotation icon, and Edit metadata icon. Finally, output components, included a base map in ArcMap document, a file geodatabase, georeferenced CAD files, and a project report.

Project was delivered successfully on time. However, the project report is not yet delivered. It is expected to deliver the report by May 2010. The expected time-duration for many tasks in the project plan was different from the reality. Thus, it caused to be a late for many tasks. 


\section{Chapter 4 - Database Design}

This chapter explains how the database was designed, the data that were used, and the methods for preparing the data for analysis. The database was designed to show how a detailed data model of a database contains detailed attributes for each feature class. Also, it describes the feature classes and tables in the logical data model for the GIS implementation. Moreover, this chapter shows where the data came from, and what metadata are important; for example, organization that prepares data, date of preparation, last date of update, how was data created, accuracy of data, projection, size and format of data. In addition, methods of collection data and responsibilities are illustrated in this chapter including the period of time taken to collect the data. Furthermore, cleaned up and prepared data before loading them into the geodatabase were explained. This chapter also discusses the challenge of dealing with enhancing the accuracy of data, and how the project scope was changed. Sections include: Logical Data Model, Data Sources, Data Collection Methods, Data Scrubbing and Loading, and Summary.

\subsection{Logical Data Model}

This section describes the feature contents in the file geodatabase with their attributes, and how they are related one another. It also explains subtypes and domains created for attributes, and selected data types for each attribute. In addition, it illustrates attribute identifications needed by the client. For more details information about data (See Appendix A for the schema report).

The file geodatabase contains two feature data sets and one raster catalog. The two feature data sets are: ground facilities and underground facilities. They contain 38 feature classes. The underground facilities feature dataset contains 18 feature classes. Most of features have feature classes and annotation feature classes include: chilled water, compressed air, condensate water, demonized water, domestic water, hot water, sewer, and steam. However, storm and tunnel features do not have annotation feature classes. The ground facilities features dataset contains 20 feature classes. Ten of them are required to identify their attributes by the client, and they are as follows: buildings, parking lots, pool areas, vegetation, streets, emergency telephones, light poles, manholes, fire hydrants, and palms. The other feature classes used for cartographic purposes, annotations, or boundaries, such as sport fields, sidewalk, parking lines, parking labels, street annotations, street polygons, tree cover, building name and number annotations, and boundaries. The logical data model focuses only on campus facility feature classes that were required to identify their attributes by the client (Figure 4.1). 


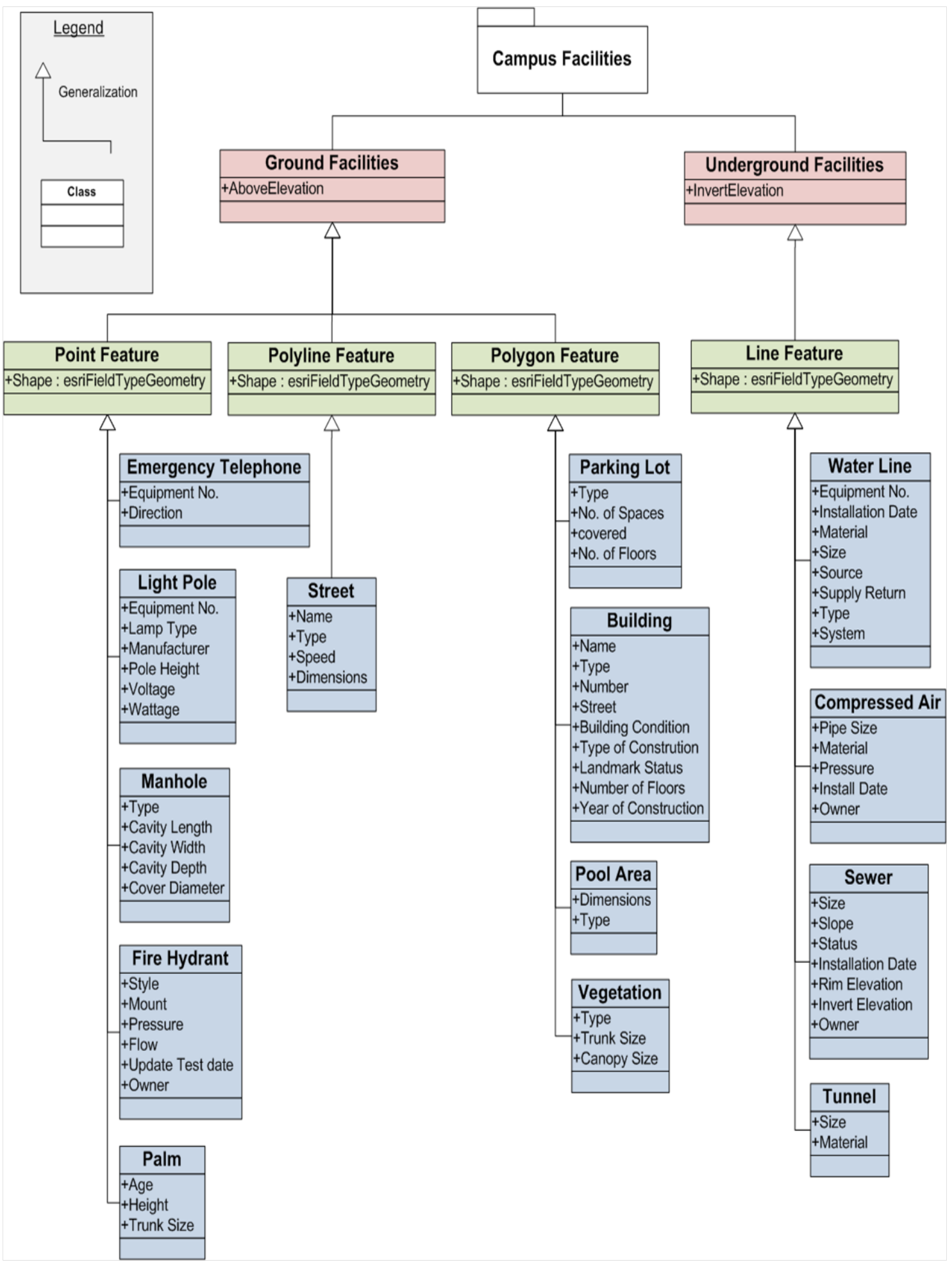

Figure 4.1: Logical Data Model 
The campus facilities are divided into two groups: ground facilities and underground facilities. Features in these groups are categorized depending on geometric type include point, line, and polygon.

\subsubsection{Ground Facilities}

Point feature classes in the ground facilities include: emergency telephones, light poles, manholes, fire hydrants, and palms. Emergency telephones were digitized using a digital map of Caltech. The rest of the point feature classes were converted from the old CAD data. The last update for these CAD files was in 1994. However, they are very accurate because they were created by using surveying measurements. Each point feature class is required to identify their attributes, and select the type of data. Also, it is recommended to create subtypes, when possible, since it provides an efficient way for editing and adding attributes, and uses less storage space in the geodatabase.

There are 18 emergency telephones on the Caltech campus. The attributes identified for emergency telephone feature class are equipment number and the direction it is facing. The data type that was selected for equipment number was text. However, eight subtypes were created for the direction field, which are north, northeast, east, southeast, south, southwest, west, and northwest. These subtypes were coded from zero to seven. Therefore, a short integer was selected as the data type for this field. When the user needs to edit or add data to the direction field, it is only required to select the attribute name instead of typing it.

There are approximately 485 light poles on the Caltech campus. They were categorized to three different sizes: large, medium, and small. There are approximately 129 large light poles, 112 medium light poles, and 244 small light poles. The large light poles usually located in main streets and sport fields while the medium one usually located in minor streets and front of buildings. The small light poles are the most common and widely distributed across the campus. The attributes that were identified for the client's needs are as follows: in a text format, there are equipment number, lamp type, and manufacturer. In a short integer format, there are voltage and wattage. In a float format, there is pole height. The reason for selecting the float format is that the number might contain decimal places with expected fractional numbers between -3.4E-38 and 1.2E38. If the expected number is bigger than that, the double format is necessarily selected. The float data type takes up to four bytes, whereas the double data type takes up eight bytes.

The third point feature is the manhole feature class. There are approximately 144 manholes around the campus. Five attributes were suggested by the client including manhole type, cavity length, width, depth, and diameter. The data type that designed for these attributes is float except for the manhole type. There are four types of manholes: sewer, electrical vault, tunnel, and telecom. Subtypes were therefore created for these types in the type field. Thus, short integers were selected for this field.

There are approximately 15 fire hydrants around the Caltech campus. Fire hydrants require annual inspections and maintenance, so the client needs to identify these following attributes: fire hydrant style, mount, pressure, flow, test update, and owner. The style means the type of hydrant: fire hydrant, post indicator valve, or Siamese connection. A post indicator controls the water flow, and a Siamese connection is a standpipe that has two connections side by side. The mount attribute indicates whether the structure is 
mounted in the wall or freestanding. In the table of attribute it is important that fire hydrant feature class contains a field for the most recent test date regarding pressure and flow to be prepared for any fire emergency. Text format was selected as the data type for fire hydrant style, mount, and owner. Regarding pressure and flow, a short integer format was selected.

The last point feature is the palm feature. There are approximately 263 palms on the campus. A palm's height and trunk size were identified as palm attributes. It is recommended that palm feature class be merged with tree feature class when it is available, and categorized as subtypes in type fields of tree attributes. It is also recommended to identify the canopy size for tree attributes.

The street feature is the only line feature in the ground facilities. Required identification attributes include: name, type, speed, and dimension. This feature was imported from ESRI street data; then streets in the Caltech campus area were selected and extracted to feature class. The default attributes by ESRI were used without any changing.

The polygon feature of the ground facilities contains four feature classes include: parking lots, buildings, pool areas, and vegetation. They were converted from most recent CAD data at Caltech. However, the feature locations of these CAD files are not accurate enough to be used for engineering purposes. Errors of plus and minus approximately ten feet were found in some areas after georeferencing the CAD files. Thus, Spatial Adjustment tools were applied to these future classes using the Rubbersheet method to provide better accuracy. Although this method minimizes the error to approximately plus or minus five feet in some areas, it is not recommended that this data be used for any engineering purposes.

There are approximately 18 parking lots in the Caltech campus. Three of them are covered and having multilevel. The attributes required to identify are parking type (covered or not), number of floors, number of parking spaces, number of handicap parking spaces, number of visitor parking spaces, number of reserved parking spaces, number of ninety minute parking spaces, and number of general parking spaces. The client suggested three types of parking lots: surface lot, Subterranean, and multilevel. Therefore, a domain for the type field was created for these types of parking lots. Also, another domain was created for the covered field with subtypes of Yes or No. The data type that selected for all identifying attributes was short integer.

Caltech has 125 buildings in a 124 acre campus. The attributes that were identified for this feature are: building name, type, number, address, condition (the physical status of the building at the time of inventory), type of construction (a code which indicates the type of construction used in the building), landmark status, number of floors, and year of construction. The landmark status indicates whether the building has a significant characteristic that includes it in any official listing, such as the National Register of Historic Buildings.

The third polygon feature in the ground facilities is the pool area feature class. There are approximately16 pool areas around the campus, and they are different in dimensions and types. Attributes that identified are name, type, and dimensions.

The vegetation feature class is a polygon geometric type in the ground facilities. The most common type is grasses. The attributes for this feature are identified to type of vegetation and trunk size. 


\subsubsection{Underground Facilities}

All underground facilities are line features, so they are categorized under the line geometric type in the logical data model. Many feature classes in the underground facilities are type of water, and share the same attribute identification, such as chilled water, condensate water, demonized water, domestic water, hot water, storm water, and steam. So they are grouped under waterline in the logical data model.

All water types require the same attribute identifications; include equipment number in text format, installation date in date format, material in short integer format, size in float format, source in text format, supply return in text format, type in text format, and system in text format. The field material can be shared by many underground facilities, such as water types, compressed air, and tunnel. Therefore, a domain was created for this field, and a short integer was selected for the data type.

There are five attributes that client required to identify the compressed air feature class: pipe size in float format, material in short integer format, pressure in float format, installation date in date format, and owner in text format. The owner is important because some pipes are owned by the city of Pasadena; and others by the California Institute of Technology (Caltech).

The sewer feature class required seven attribute identifications, including sewer size in float format, slope in float format, status in text format, installation date in date format, rim elevation in float format, invert elevation in float format, and owner in text format.

Finally, the client required two attributes for tunnel feature class, which are tunnel size and material. The data type for tunnel size is a float, and for material is a short integer because a domain was created for the material field.

\subsection{Data Sources}

Data in this project include: CAD files, aerial photos, and street shape file. The Facilities Department at the Caltech provided CAD files, and limited to 13 files in DWG format. Features in these CAD files differ in accuracy and updating date. One, called 100TOPO, is more accurate than the others because it was created by ASL Consulting Engineering using surveying measurements. However, the last update was in 1994. Thus, only layers that were most needed and not provided from other CAD data were extracted from the 100TOPO CAD file, include light pole, manhole, fire hydrant, and palm layers. The other CAD files were created from a photo that was not orthophoto. Also, surveying measurements were not used in the initial creation of CAD features. Although the locations for the CAD feature are not accurate, they are often used and updated. These CAD files are Chilled Water, Compressed Air, Condensate Water, Demonized Water, Domestic Water, Hot Water, Sewer, Steam, Storm Water, Tunnel, All Utilities, and XCAMP-MAP-a. Four feature classes were extracted from the XCAMP-MAP-a CAD file including parking lots, buildings, pool areas, and vegetation. The city of Pasadena provided nine aerial photos that cover 5.8 square kilometer with 0.1 meter resolution. These aerial photos were taken in the winter of 2006. Each aerial photo has a size of 183 mega bytes in a TIF format, and the projected coordinate system is NAD_1983_StatePlane_California_V_FIPS_0405_Feet. 


\subsection{Data Collection Methods}

The data collection task followed the requirement analysis and planning task. The methods of data collection were discussed and planned in the first meeting with the client during the requirement analysis and planning task. The client took responsibility for providing clean CAD files. The aerial photos were requested from the city of Pasadena. It took one month for aerial photos to be received. The street shape file was exported from the ESRI folder in the K drive that belongs to the University of Redlands.

\subsection{Data Scrubbing and Loading}

The project was spent extra time on cleaning up CAD files. Before loading CAD files into the geodatabase, $\mathrm{CAD}$ files were required to assign spatial reference, georeferencing, and extract useful layers. The first group of CAD files provided by the client contained more than 500 files. Each drawing was given a codename, so it was difficult to tell what the drawings represented. Moreover, the features of these files had errors of more than plus or minus five feet. The client required a minimum accuracy of plus or minus two feet, which was impossible to provide since the original CAD features had less location accuracy than that. This bad accuracy was due to one of two reasons: either the aerial photos are not accurate, or the CAD features are not accurate. To check the accuracy of the aerial photos, another aerial photo that covered the same project area was downloaded from a seamless website. It was then overlaid with the original aerial photos. By using the Swipe Layer button from the Effects tool, it made it easy to see what was underneath the seamless aerial photo layer without having to turn it off in the table of contents. This test showed that the same places in the two aerial photos were exactly overlaid. Another test also was applied; some ESRI feature classes such as street were added to the aerial photo layer. It was found that the street feature class overlaid with the aerial photos in the correct places. The aerial photos were accurate enough to provide better accuracy than plus or minus two feet. Therefore, the client was told that the CAD features were not accurate enough to provide the required accuracy of plus or minus two feet. The client suggested sending another group of CAD files which were not provided before and that were different in accuracy than previous provided group of CAD files. The newly provided group of CAD files was created by using surveying measurements. After testing the accuracy of them, it was found to be accurate enough to provide the required accuracy of plus or minus two feet. Unfortunately, the last date of update for these newly provided of CAD files was 1994, and few drawings were found to be useful for using in this project, such as light pole, manhole, fire hydrant, and palm. Other files were taken from inaccurate CAD files such as parking lot, building, pool area, vegetation, and underground facilities. Thus, it was better to enhance their accuracy before they could be used. The way to do that was by applying the Rubbersheet method from the Spatial Adjustment tool. The inaccurate CAD features lost their geometric accuracy because they were created from a photo that not orthophoto. So, geometric distortions were different in different locations. The Rubbersheet adjustment was used to make geometric adjustments in the CAD features by aligning them with aerial photos. 


\subsection{Summary}

The logical data model was implemented for designing the geodatabase. The file geodatabase has two feature data sets for campus facilities and one raster catalog for aerial photos. The feature classes were categorized in the logical data model depending on the geometric type, and whether if they were ground or underground facilities. To minimize the storage of the file geodatabase and improve the performance, domains and subtypes were created for some attributes, and a data type was selected for each attribute. Only specific feature classes with their attributes, which were identified by the client, show in the logical data model.

Provided data has three different sources. The city of Pasadena provided aerial photos, street shapefile was downloaded from ESRI, and the client provided CAD files. These files came in two groups that different in accuracy and the last date of update. The most current one had bad accuracy which is more than + or -5 feet in some areas. The Rubbersheet method in the spatial adjustment tool was applied to enhance the location accuracy for CAD features. The second group of CAD files was the older one that has good accuracy. Since the last date of update was more than 14 years, only necessary layers were extracted from these CAD files. 



\section{Chapter 5 - Implementation}

This chapter discusses the implementation for the project in detail so that any GIS professional can replicate the work. The implementation was done in six steps, including: georeferencing CAD files, extracting needed CAD data layers, geodatabase design, applying spatial adjustment, base map creation and design, and converting feature classes to CAD files.

\subsection{Georeference CAD Files}

The georeferencing CAD data implementation included assigning spatial references for CAD files, building pyramids for aerial photos, applying the Georeferencing tool, and testing the accuracy of aerial photos.

\subsubsection{Assign Spatial Reference for CAD Files}

The CAD files lacked any information defining which coordinate system was used to create the features. It is possible to work with data even if they do not have a spatial reference. However, in order to take advantage of all GIS functionalities, data must assign a spatial reference. Moreover, data that need to be georeferenced must have the same spatial reference for aerial photos. Thus, data were assigned the same spatial reference as the aerial photos by importing the spatial reference for one of the aerial photos using ArcCatalog. When the coordinate system was defined, it was stored in a .prj file with the same filename as the data and in the same folder.

\subsubsection{Build Pyramids for Aerial Photos}

When aerial photos were added to ArcMap, a Dialog box popped up and asked for creating pyramids for aerial photos. This Dialog box only displays with a raster dataset larger than 1024 by 1024 cells, but each aerial photo had 8000 by 8000 cells. Thus, pyramids were built for all aerial photos to speed up the display of aerial photos. However, build pyramids can be created for any size. To build a pyramid, select the raster from ArcCatalog, then right click and click Build Pyramids. After building pyramids, the information is stored in a reduced resolution dataset (.rrd) file format in the same folder as the aerial photo and the same filename. "Pyramids are copies of the original bands of data in which representations of a dataset are reduced by multiples of 4, so the computer returns the data at a specified resolution required for the display” (ESRI, 2008).

\subsubsection{Apply the Georeferencing Tool}

After assigning a spatial reference for CAD files, they were added to ArcMap with aerial photos for georeferencing. Then the Georeferencing tool was added to the main menu, and a CAD layer that needed to be georeferenced was selected in the Layer box in the Georeferencing tool (Indicated by number 1 in Figure 5.1). Zoom To Layer was then applied for the aerial photos, so CAD features could display with aerial photos after selecting Fit To Display in the Georeferencing tool (Indicated by number 2 in Figure 5.1). 


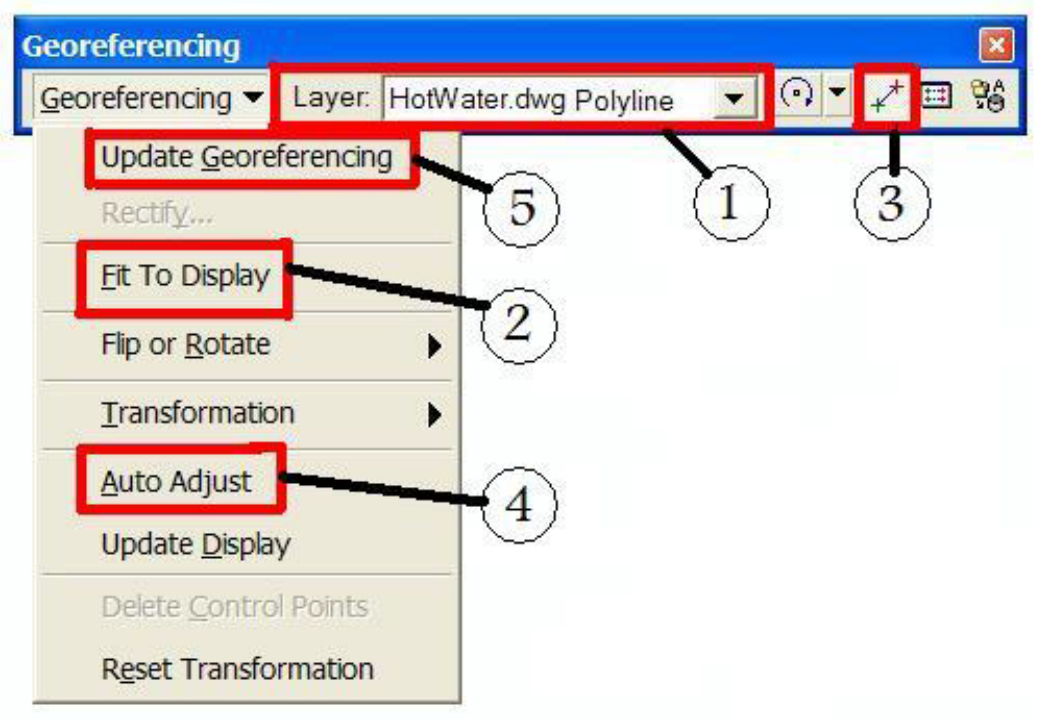

Figure 5.1: The Georeferencing Tool

This tool provides only two control points for georeferencing CAD files because $\mathrm{CAD}$ transformations are based on the similarity transformation method that maintains relative shape and the aspect ratio of $\mathrm{CAD}$. Thus, two control points were added to define the transformation by clicking the Add Control Points tool (Indicated by number 3 in Figure 5.1). The first mouse click for the first control point defined the source, which was from CAD coordinate. The second mouse click defined the destination, which was to aerial photo coordinates. After creating the two control points, CAD features were adjusted by clicking the Auto Adjust tool (Indicated by number 4 in Figure 5.1). These two steps were repeated until CAD transformation was satisfied. Finally, georeferencing was updated by clicking Update Georeferencing in the Georeferencing menu list (Indicated by number 5 in Figure 5.1). In the Save As Dialog box, the name must be the same for the CAD dataset's name and in the same folder. Once the world file was saved, the CAD layer was transformed.

\subsubsection{Test the Accuracy for Aerial Photos}

After georeferencing CAD files, it was found that the CAD features had errors of more than plus or minus five feet in some areas. These errors were different in number and direction from area to area on the campus, as shown in Figure 5.2. The reason was that either the CAD features or the aerial photos were not accurate. Therefore, an accuracy test was applied to the aerial photos. 


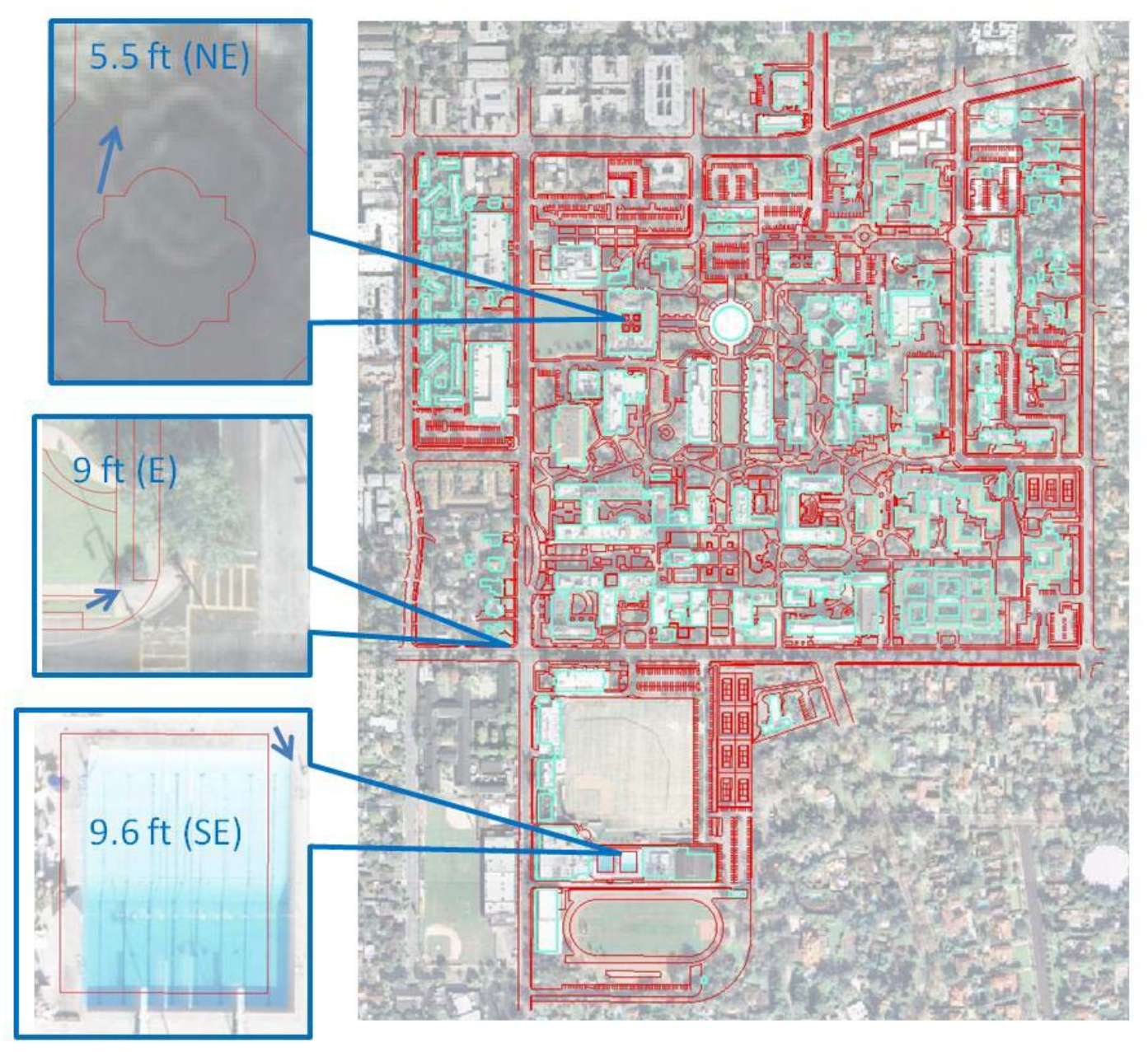

Figure 5.2: Location accuracy after georeferencing CAD features

The accuracy of aerial photos was tested by downloading another aerial photo from a different source (www.seamless.com) for the same project area. It was then added to ArcMap with aerial photos that needed to be tested. The Swipe icon in the Effects tool was used to interactively reveal what was underneath the Seamless aerial photo to make sure that the aerial photos were overlaid with it in all project areas. Moreover, a street shape file was downloaded from ESRI and added to ArcMap with aerial photos to double check that they were also overlaid with the aerial photos in their correct place. After applying these two accuracy tests to the aerial photos, it was found that they were accurate enough to provide a minimum accuracy of plus or minus two feet. Therefore, the problem of the bad accuracy must be caused by the CAD features. The client was informed about the CAD accuracy problem, and subsequently decided to provide another group of CAD files created by different source. When this second group of CAD files was georeferenced, it was found to be more accurate than the previous group, and suitable for the client's needs. However, the date of the last update for this second group of CAD files was 1994, and unfortunately most of its layers were found not to be unnecessary and not useful for this project. Thus, many layers from the inaccurate CAD 
data were added and used. The first group of CAD files had bad accuracy in their feature locations because they were created from a photo that was not an orthophoto. Also, surveying measurements were not applied when these CAD features first created, which caused geometric distortions. Therefore, the spatial adjustment was applied to enhance the accuracy by using the Rubbersheet method (Section 5.4). However, CAD data should be converted to feature classes or shapefiles in order to apply the spatial adjustment. Thus, this step came after creating feature classes.

\subsection{Extract Needed CAD Data Layers}

The CAD data contained many layers, and not all of them were useful for this project. Therefore, only the necessary CAD data layers were extracted and converted to the feature class. The first step for extracting CAD data was to select the geometric type of needed CAD data: point, polyline, polygon, or annotation. Next, from the Drawing Layers tab in the Layer properties for the selecting geometric type, only one of needed drawing layers was checked. It was then copied and pasted in the data layer frame. This step was repeated for all needed layers one by one. For example, the underground facilities CAD file had more than 50 layers. Only 11 layers were needed from the polyline CAD data, however, each of these layers was checked separately. They were then copied and pasted in the data layer frame one by one (Figure 5.3)

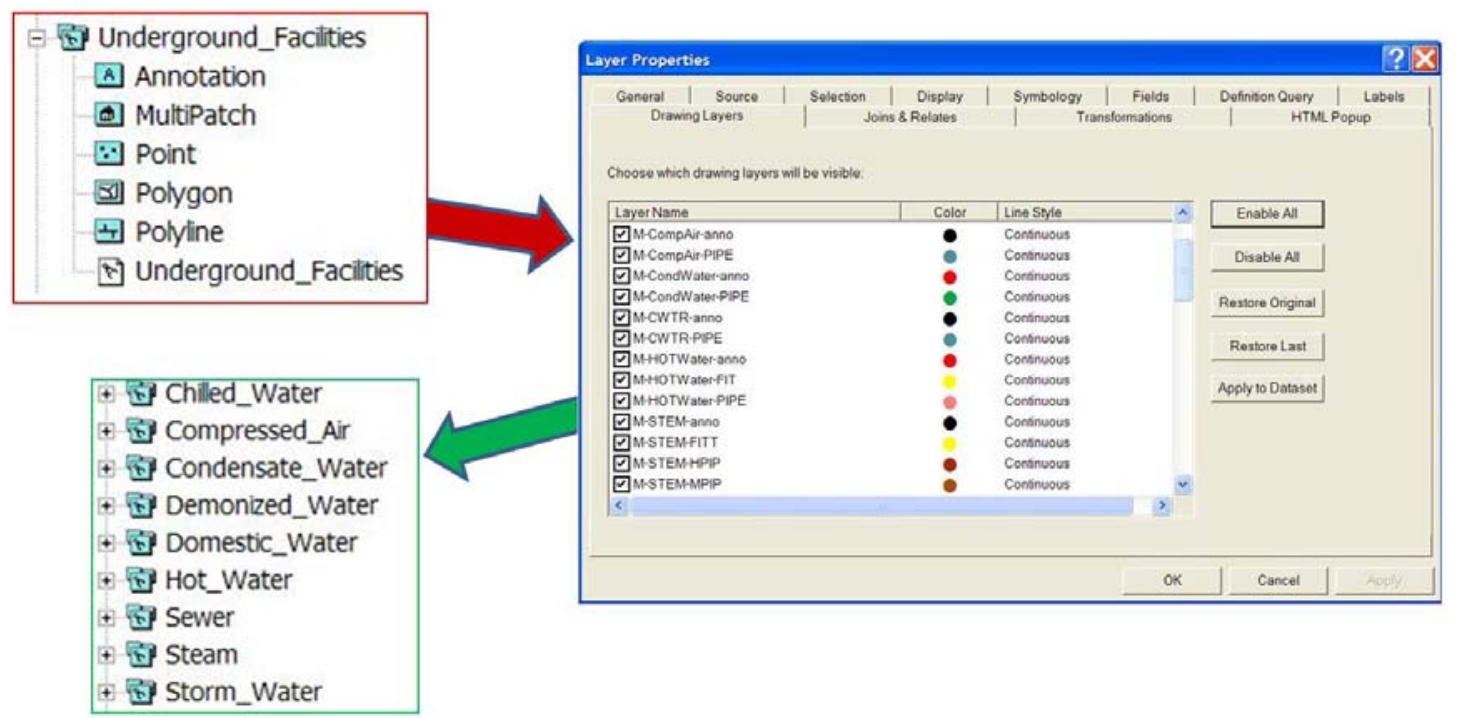

Figure 5.3: Extract needed CAD data layers

Some necessary CAD data layers, however, could not be extracted because these drawings were block entities, a single object. Don Kuehne, the technical product manager for CAD interoperability at ESRI, suggested exploding drawings into their individual features using AutoCAD in order to fix the problem. This problem occurred because someone inserted one drawing into another, so the entire inserted drawing turned into a single object. Thus, David Mispagel, a Caltech Project Manager, could explode the drawings into their individual features, and provide them to be used in the project. 


\subsection{Geodatabase Design}

Geodatabase design for the project involved converting CAD data to geodatabase feature classes, organizing feature classes and raster data sets, applying topology rules, identifying attributes for feature classes, creating subtypes and domains, and creating metadata.

\subsubsection{Convert CAD Data to Geodatabase Feature Classes}

A file geodatabase holds dataset files in a file folder up to one terabyte in size and only a single editor is needed. Thus, a file geodatabase was created. Then CAD data were exported to geodatabase feature classes. This can be done in ArcMap or ArcCatalog. In ArcCatalog, right click in the file geodatabase and select import. In this project, necessary CAD data were exported to the file geodatabase using ArcMap by right clicking for each CAD data and hitting Export Data. However, exporting annotation was different from other geometric types. It required converting it using Convert Coverage Annotation tool (Figure 5.4). This tool is in Customize from Tools in the main menu. Customize has many commands in the Commands tab. The tool is usually located in Label from Categories list. Many annotations can be converted at once to geodatabase annotations by using this tool. ArcCatalog must be closed in the process of converting the annotations in ArcMap. Otherwise, an error Dialog box will pop up.

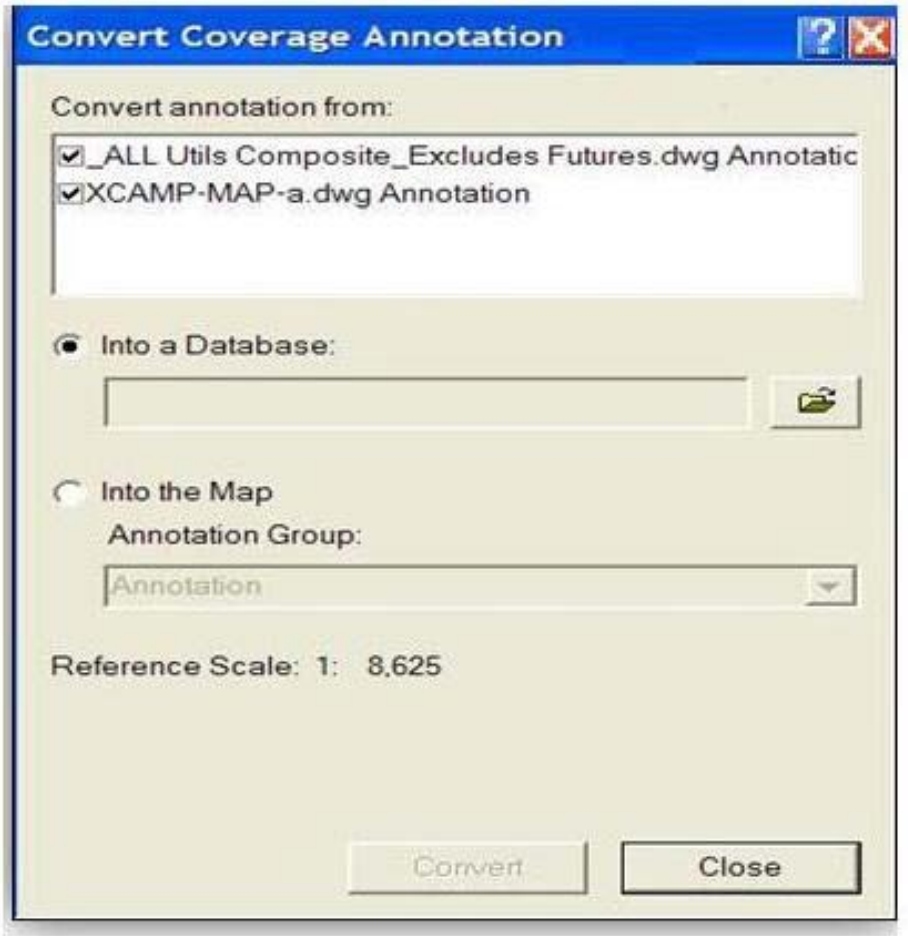

Figure 5.4: Convert Coverage Annotation tool 


\subsubsection{Organize Feature Classes and Raster Data Sets}

The file geodatabase contains feature classes and raster data sets. Feature classes in the project were categorized as ground facilities and underground facilities. Thus, two feature data sets were created: one for ground facilities and another for underground facilities. Moreover, building topology (section 5.3.3) required related feature classes to be in a common dataset. Also, there are many other uses of feature data sets such as building network dataset, geometric network, terrain, and cadastral fabric. However, in this project, building topology was only used for feature data sets. Raster data sets were the aerial photos, and they were eight raster data sets. A raster catalog was created as a container for these raster data sets in the file geodatabase. In the raster catalog, raster data sets displayed as a single layer.

\subsubsection{Apply Topology Rules}

Some features, such as buildings and tree cover, were converted from polyline in CAD to line features in GIS. These features were not converted to polygon immediately because some features had non-closed boundary objects, and only objects with enclosed boundaries could be converted to polygon (Figure 5.5).

\section{buildings}
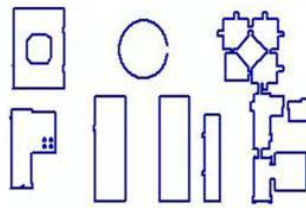

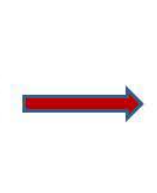

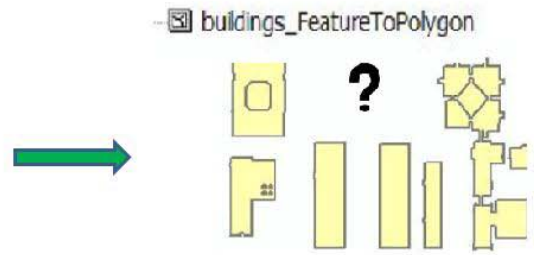

Figure 5.5: Feature to Polygon tool converts only enclosed boundary objects

There were two ways to fix non-closed boundary objects: performing digitizing or applying topology rules. Both ways were tried. Digitizing was performed on non-closed objects that could be easily found and seen. Two topology rules were applied: Must Not Intersect or Touch Interior, and Must Not Overlap (Figure 5.6). The cluster tolerance was adjusted to three feet, which means vertices that fell within the cluster tolerance were snapped together during the validate topology process. If a value of zero was entered, the default or minimum cluster tolerance applied to the topology. After validating topology and checking that all objects had closed areas, the Feature To Polygon tool in the Data Management Tools was applied to convert the line features to polygon features for buildings and tree cover. 


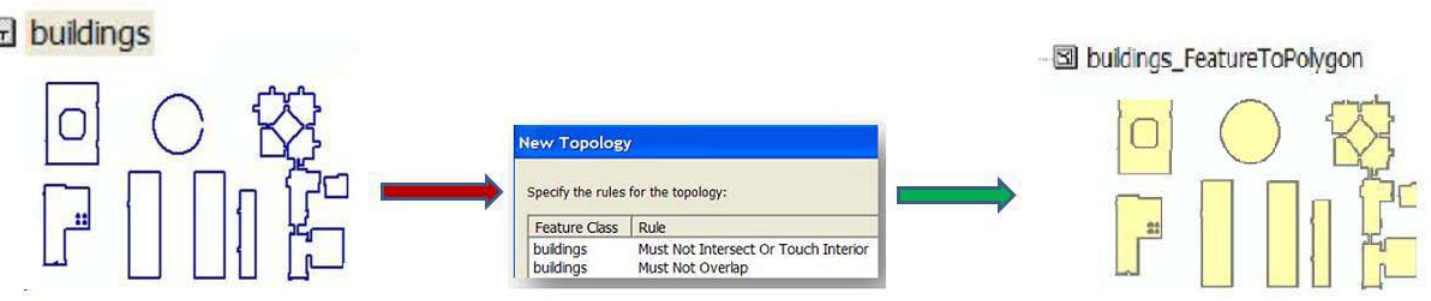

Figure 5.6: Convert features to polygons after applying topology rules

\subsubsection{Identify Attributes for Feature Classes}

Attributes that need to be identified for feature classes should have been known before starting project implementation so that the client had enough time to collect the necessary information. However, due to the lack experience, identifying attributes with the client came during the geodatabase design implementation. Therefore, attributes in the project were identified without adding their information. Attribute tables for feature classes include CAD properties in their attributes. They came from CAD data conversion to feature classes. When CAD files are moved to GIS, CAD properties are treated in ArcGIS as attributes of CAD features. These properties were descriptions of CAD features such as line color, line width, line type, thickness, etc. Therefore, CAD properties in the attribute table for feature classes were deleted because they were not useful to the GIS. They were deleted in ArcCatalog from the Fields tab in the Feature Class Properties. This was also a way to add attributes identified by the client. Each field name was required to select one data type. There were nine data types available to be chosen, including: Short Integer, Long Integer, Float, Double, Text, Date, BLOB, GUID, and Raster. According to ArcGIS 9.3 Desktop Help, if data is needed to store integers only between - 32,768 and 32,767, the short integer should be specified because a short integer takes up only two bytes, whereas a long integer takes up four bytes. If data is needed to store fractional number between $-3.4 \mathrm{E}-38$ and $1.2 \mathrm{E} 38$ only, the float should be specified because it takes up four bytes, whereas the double takes up eight bytes. The date data type can store dates, times, or both. Choosing the correct data type minimizes the storage of the file geodatabase and improves the performance. It also facilitates analysis, data management, and business needs (ESRI, 2008). The binary larger object (BLOB) and global identifier (GUID) data types were not used for this project because there was no need for long sequences of binary numbers or applications requiring globally unique identifiers. Finally, text represented a series of alphanumeric symbols. The expected maximum length for each text attribute was identified in the field properties table.

\subsubsection{Create Subtypes and Domains}

Subtypes and domains speed data entry and ensure that it remains accurate and validates data stored in the geodatabase. This has the advantage of providing an efficient way for editing and adding attributes, and using less storage space in the geodatabase. 
Subtypes categorize records within a field by specifying an integer code and an associated description to provide only valid attribute values for that field. For example, in the project there were four types of manholes: sewer, electrical vault, tunnel, and telecom. So subtypes were created for the Type field. A short integer was selected for this field as data type. Then from the subtypes tab, the type field was given integer codes values for its attributes (Figure5.7). The code 0 was given for the sewer pipes, code 1 for electrical vault, code 2 for tunnel, and code 3 for telecom. When the user wants to add or edit an attribute in the type field, the user just needs to select between these subtypes instead of typing them.

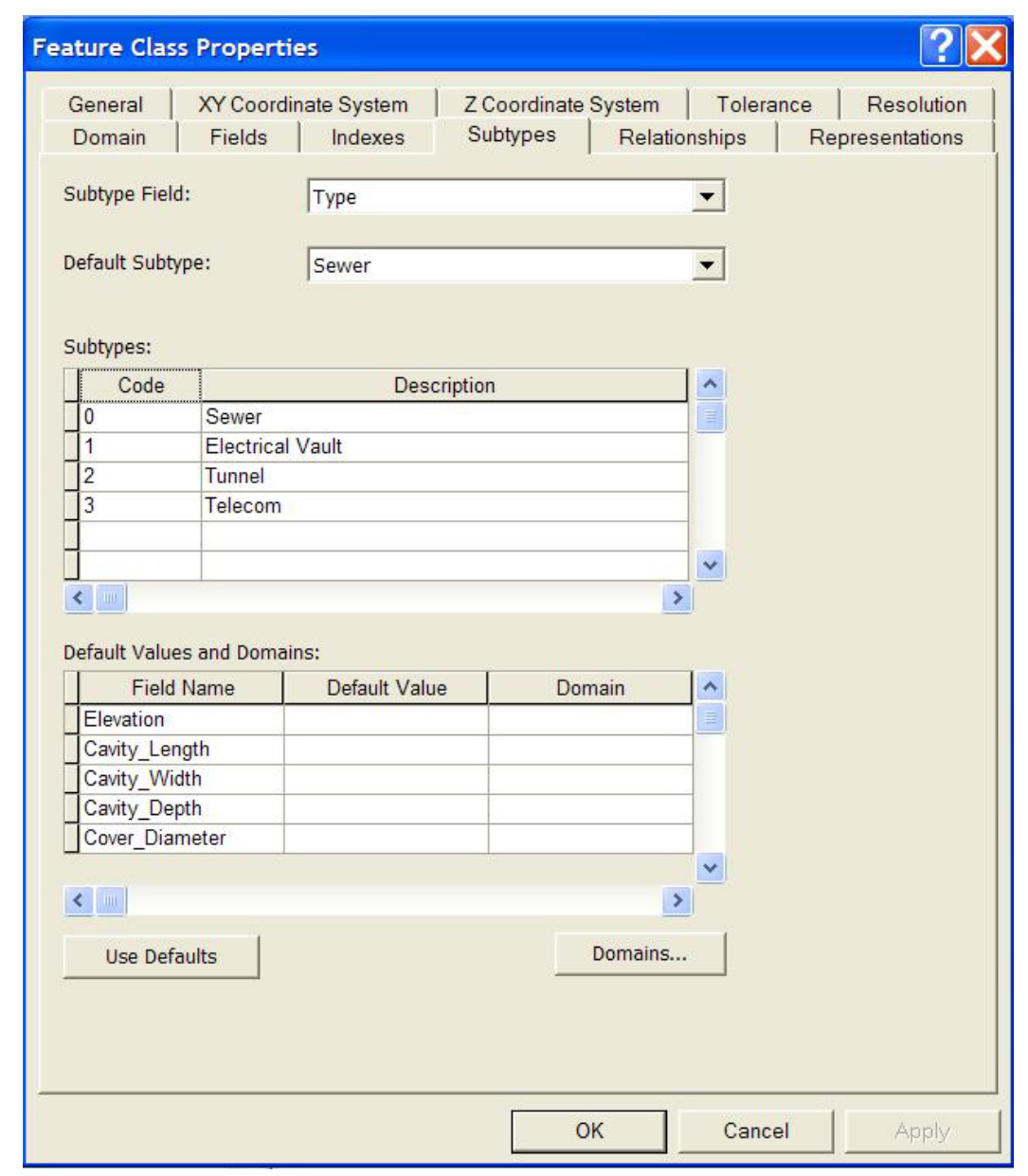

Figure 5.7: Creating a subtype for manhole feature class

Domains control valid attribute values for any field in the geodatabase. They can be shared for any feature classes. There are two types of domains: coded value domains and range domains. A range domain is a numeric attribute that specifies a range of numeric data entry with minimum and maximum values. It is used for preventing data entry error. A coded value domain can be used for any type of attribute to specify a valid set of values. For example, in the project, there were many underground facility feature classes that had a material field. The choices of the material field were the same for these underground facilities. Thus, a coded value domain called materials was created for these 
feature classes. In addition, a feature class cannot have more than one subtype. However, domain attributes can be created instead. For example, the parking lot feature class needed two different subtypes: one for the Type field, and another for the Covered field. For creating a domain, the Domains button was clicked to open the workspace domain (Figure 5.8). In the workspace domain there are three separates tables: domain name and description, domain properties, and coded values. The domain properties table has four properties: Field Type, Domain Type, Split Policy, and Merge Policy. Split and merge policies control attribute values when a feature is split or merged. In the project, the split policy and merge policy were applied to the default, which means the attribute of the resulting feature took on the default value for the attribute of the given feature class or subtype. However, it was found that some attribute domains in the file geodatabase were automatically generated. These domains were AnnotationStatus, BooleanSymbolValue, HorizontalAlignment, and VerticalAlignment. They support feature linked annotation. "The AnnotationStatus indicates whether a feature has annotation or not. The BooleanSymbolValue domain indicates if user defined symbols are utilized. The HorizontalAlignment and VerticalAlignment domain store the position for feature linked annotation" (Addison, 2006). For more information about domains and subtypes in the project (See Appendix A).

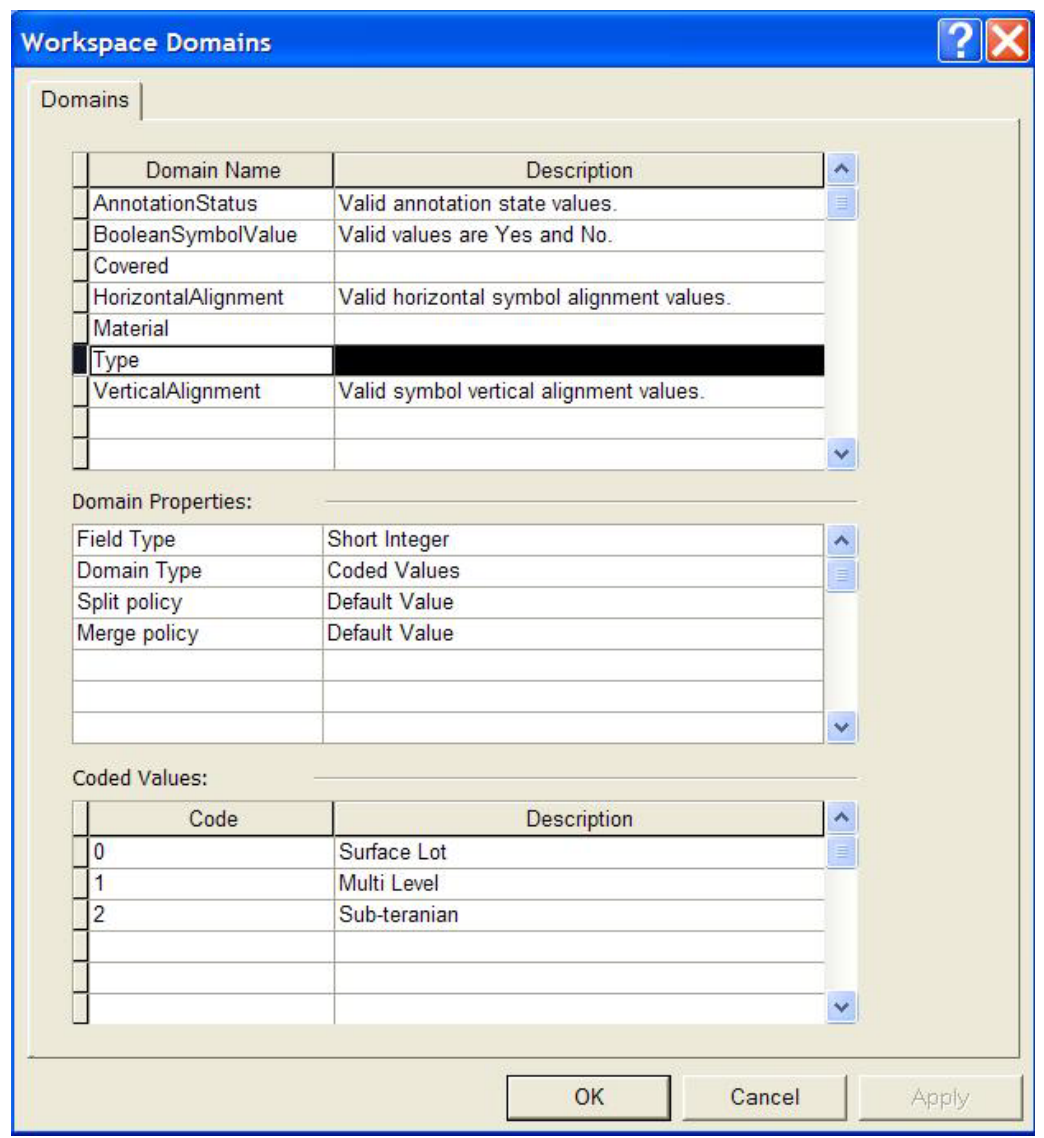

Figure 5.8: The project Workspace Domains 


\subsubsection{Create Metadata}

The last task in the geodatabase design was creating the metadata for feature data sets, feature classes, aerial photos, file geodatabase, and CAD data. This was done using the Edit metadata icon in ArcCatalog. To make that icon active, the metadata tab must be clicked. The style sheet used for metadata was FGDC ESRI. Metadata for all data included: abstract, purpose, point of contact for the person or organization that provided data, date for creating metadata, keywords for theme and place, data quality report, data accuracy, date of data creation, spatial reference, and contact information for the person who created metadata. Contact information included name of person, position, organization, telephone number, and address. Metadata was stored in XML format.

\subsection{Spatial Adjustment}

The first group of CAD files provided by the client, which is the most recent files, was created from a photo that was not an orthophoto. Also, surveying measurements were not applied when these CAD features first created. Thus, these CAD features had bad accuracy in their locations, and provided errors of plus or minus approximately ten feet for feature classes that were converted from them. Accuracy can, however, be enhanced by applying the Rubbersheet method from the Spatial Adjustment tool due to irregular geometric distortions. This method enhances the accuracy by aligning unreferenced features to more accurate information derived from orthophoto. The following is a brief description of how the spatial adjustment process was done. The inaccurate CAD data was first converted into feature classes. These feature classes were added onto ArcMap, and an edit session was started from the Editor tool to make the Spatial Adjustment tool active. Then snapping and snapping tolerance were adjusted from the Editor tool. Next, input for adjustment was chosen from Set Adjust Data in the Spatial Adjustment tool menu. All inaccurate feature classes were chosen for spatial adjustment. The Rubbersheet method was selected from Adjustment Method in the Spatial Adjustment tool menu. According ArcGIS 9.3 Desktop Help, the Rubbersheet method uses a piecewise transformation that preserves straight lines to move features (Figure 5.9). It uses two temporary triangulated irregular networks (TINs) like structures to interpolate changes in the $\mathrm{x}$ and $\mathrm{y}$ axis for source (from) and destination (to). A node is defined by its $\mathrm{x}, \mathrm{y}$ location and a $\mathrm{z}$ value which is used to interpolate the amount of adjustment, unlike the $\mathrm{z}$ value that used for elevation, as in a standard TIN. The $\mathrm{z}$ value is the amount of change between the from-end and to-end of a link (ESRI, 2008). 


\section{Target layer (accurate)}

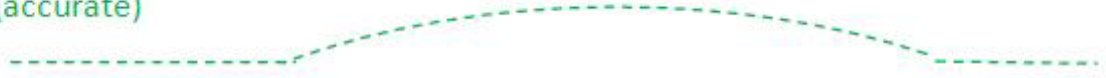

Source layer (inaccurate)

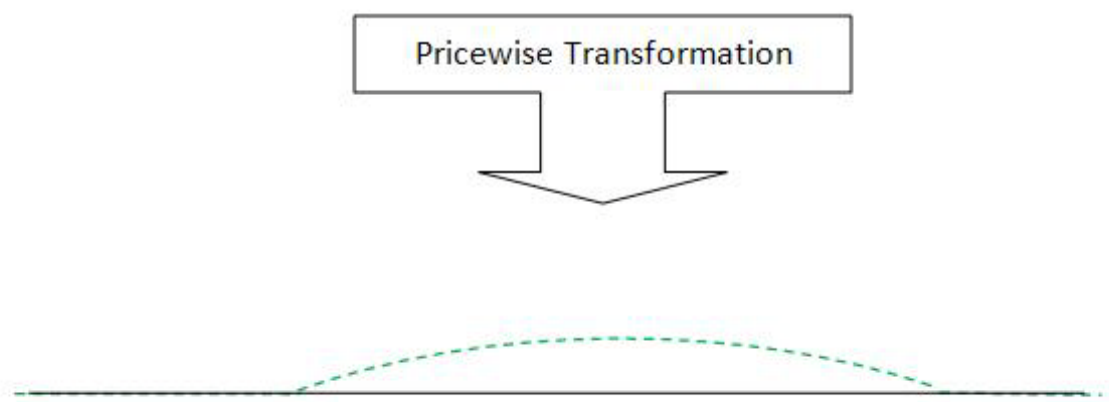

Figure 5.9: Piecewise transformation

The Rubbersheeting adjustment has two different interpolation methods: linear and natural neighbor. According to ArcGIS 9.3 Desktop Help, the linear method is quick and accurate if there are lots of displacement links over the being adjusted, but it does not account for the neighborhood. The natural neighbor is similar to Inverse Distance Weighting (IDW). It is slower but more accurate if there are not many displacement links (ESRI, 2008). In the project, displacement links were scattered across the project area, so the natural neighbor method was more accurate to use than the linear method. Therefore, the natural neighbor option was chosen and new displacement links were created from the New Displacement Link icon in the Spatial Adjustment tool. The first mouse click was in the feature class source. The second mouse click defined the destination, which was the aerial photo. Many displacement links were created. The closer features were to displacement links, the further they moved. However, some locations matched the target. They did not need to create a displacement link, so another type of link called an identity link was created, which is made for such issues. After creating many displacement links and identity links, they were adjusted by clicking Adjust in the Spatial Adjustment tool menu. Finally, the edits were saved from the Editor tool.

\subsection{Base Map Creation and Design}

The base map was created and designed for the California Institute of Technology (Caltech) to be used for facilities management purposes by the Facilities Department. The base map contained fundamental features for the campus, such as streets, buildings, emergency telephones, fire hydrants, light poles, manholes, parking lots, pool areas, sidewalks, sport fields, vegetation, aerial photos, and underground facilities. Underground facilities included chilled water pipes, hot water pipes, compressed air pipes, condensate water pipes, demonized water pipes, domestic water pipes, sewer pipes, 
steam pipes, storm water pipes, and tunnels. The campus streets were selected and extracted from the ESRI street shapefile. Emergency telephones were digitized to create their feature class. Their locations were estimated by using an emergency telephone digital map for Caltech. In the same way, some pool areas were digitized because the provided map had not updated since 1994. Applying the same cartography that the Facilities Department uses helps users in the department to be familiar with design of the base map. Thus, many created feature classes applied the same cartography for the digital map of Caltech, such as vegetation, sidewalks, parking buildings, annotations, parking lines, and sport fields. The sidewalks were created by applying the Erase tool from the Analysis tool. The input was boundaries, and erase features were vegetation, streets, buildings, pool areas, and sport fields. The Erase tool accepts only one erase feature, so the tool was applied many times to create the sidewalks feature class. Parking lots and parking lines were selected and their feature classes were extracted. Cut Polygon Features and Merge tasks in the Editor tool were applied to create the parking lots features.

The next step was design the base map (Figure 5.10). The base map design included assigned symbols, specified range of scales, creating and designing labels, and designing layout and map elements. Feature classes were divided into three groups in the table of contents: ground facilities, underground facilities, and annotations. The ground facilities group had one subgroup called Display on Min. Scale of 1:2000. This subgroup was created for ground feature classes that only display on a minimum of scale 1:2000. The reason for that scale was due to the difficulties to see more detailed information out beyond 1:2000, and prevent the map from being too busy. These features were palms, manholes, light poles, and fire hydrants. Annotations were grouped to two subgroups: ground facility annotations, and underground facility annotations. 


\section{CALIFORNIA INSTITUTE OF TECHNOLOGY CAMPUS MAP}

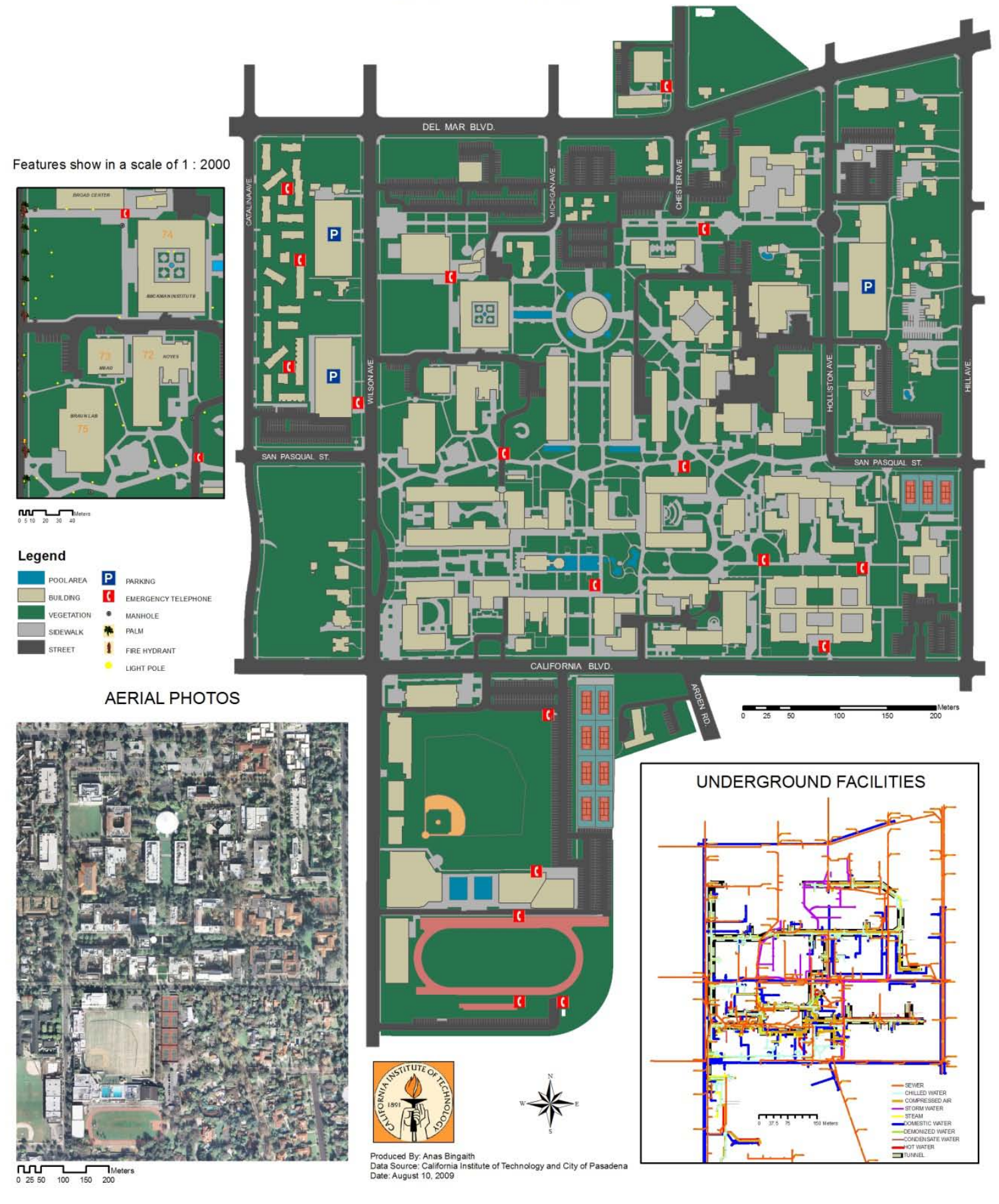

Figure 5.10: Caltech base map contents 


\subsection{Convert Feature Classes to CAD Data}

The last step of the implementation was converting feature classes to CAD data. Some CAD data had bad accuracy in their feature classes. The way to enhance their accuracy was to converting them to feature classes, and then apply the Spatial Adjustment tool. Some CAD data also had polygons with non closed boundaries. This problem was fixed by applying the topology and digitizing for feature classes. Therefore, feature classes were converted to CAD data so that the client has better accuracy of CAD feature locations, and CAD data with enclosed areas including their ideal geometric type. The method of converting feature classes to CAD data was done using Export to CAD tool in the tool box. The output type was set to the same DWG format as the original one. 


\section{Chapter 6 - Results and Analysis}

This chapter presents the results and analysis of the project. It also explains how this project was important to the client and what major problems were faced. This project is the beginning of smooth transition from CAD to GIS for the client, and providing an excellent environment for spatial data sets. Moving from CAD to GIS involved georeferencing CAD files, converting CAD data to geodatabase feature classes, geodatabase design, and base map creation. These tasks were useful for the client in assigning geographic coordinates to spatial data, providing better management of data, base map creation and design, and accuracy enhancement of CAD feature locations.

\subsection{Assigning Geographic Coordinates to Spatial Data}

The project assigned a reference and geographic coordinates to spatial data by georeferencing CAD files. This provides the advantage of using all the functionality of a GIS, and relating spatial data to a map from many sources in a global coordinate system. For example, if the city of Pasadena decides to provide recent sewer data, including drawings and attributes, to the Facilities Department at the Caltech, users at Caltech could relate these data to the base map in effective and efficient way by using GIS.

\subsection{Providing Better Management of Data}

GIS provides solutions for data management. This project allows users to employ all the functionality of GIS and its tools to provide better management of facilities. These tools are used to manage and organize various data sets, as well as to display, explore, analyze, assign symbols, print, publish, and create maps. Data in this project were organized, documented, identified, assigned subtypes and domains, cleaned, and enhanced.

All data were stored in one container: a file geodatabase. They were categorized to feature data sets and raster catalog, so users do not need to search for data from different places and formats. This saves time searching for data, and prevents duplication of work. For any project, information about data is required to search different formats and sources. For example, users at Caltech need to search from an index book to figure out the codename that given for each CAD file.

Data sets used by the Facilities Department at Caltech were lacking metadata. Therefore, the project provided metadata for each dataset. Metadata included properties and descriptive information about the data. GIS stores this valuable document in one place and format. This reduces time spent searching for information about data and resources. For example, when the client provided CAD files, they did not include any important information about them, such as date of collection, quality, and how they were created. Lack of metadata made users in the Facilities Department to work and update the wrong group of CAD file that has bad accuracy in their feature locations. However, the CAD files that have most accurate features were ignored and had not updated since 1994. Furthermore, the lack of metadata caused more time in the project for testing the accuracy of aerial photos and figuring out what the causes of bad accuracy were after georeferencing the $\mathrm{CAD}$ files. 
Attributes and properties of geographic objects were identified in a table so that geographic features could be visualized, queried, and analyzed from information in the table of attributes. Some of these attributes were assigned subtypes and domains to provide an efficient way for editing and to use less storage space in the geodatabase.

The project provided clean data sets. Non useful CAD properties in the table of attributes were deleted. Incomplete drawings were digitized, or applied some topology rules. For example, many polygon features in CAD data such as buildings and tree cover had non closed boundaries. Therefore, enclosed polygons were provided to the client, so areas and polygon topology could be applied by users. Features in a GIS have relationships between them and appear they understand each other. Thus, users can create networks and topology. Users could also recombine any features to provide better data.

\subsection{Base Map Creation and Design}

The project provides a base map to the client. Data sets in the base map came from many sources. Aerial photos were provided by the city of Pasadena, and provided to the client. The street feature class was created by ESRI and downloaded from the University of Redlands. Streets in the project area were then extracted and added to the base map. Many feature classes were converted from CAD layers, such manholes, fire hydrants, palms, light poles, pool areas, buildings, parking lots, sport fields, tree cover, vegetation, sidewalks, and all underground facilities. Underground facilities include hot water, chilled water, sewer, tunnel, storm, steam, compressed air, condensate water, demonized water, domestic water, and condensate water. However, some of these feature classes were enhanced and digitized to provide better data to the client, such as pool areas, buildings, sidewalks, parking lots, sport fields, and tree cover. Moreover, the emergency telephones feature class was created by digitizing. Different maps were created for Caltech campus as follow: Ground facility maps included buildings, vegetation, light poles, parking lots, manholes, and fire hydrants (Appendix B). Underground facility maps include hot water, demonized water, domestic water, compressed air, steam, chilled water, condensate water, sewer, tunnel, and storm (Appendix C). The features classes were assigned symbols and labels. Some features were designed to display in different scales, so it prevents the map from being too busy.

\subsection{Accuracy Enhancement of CAD Feature Locations}

The major problem in the project was the bad accuracy of some CAD feature locations provided by the client, which produced inaccurate feature classes. However, the rubbersheet method was applied to enhance the accuracy and provided better accuracy of feature classes. Thus, feature classes were converted to CAD data after rubbersheeting to provide better accurate of CAD feature locations to the client. 


\section{Chapter 7 - Conclusions and Future Work}

\subsection{Conclusion}

This project begins the process of adopting GIS to work with CAD for the Facilities Department at the California Institute of Technology (Caltech). Procedures and methodologies for this project were provided to the Facilities Department so that any GIS professional user can replicate the work. These procedures and methodologies include: georeferencing CAD files, geodatabase design, spatial adjustment, and base map creation and design. The project is useful for the client in assigning geographic coordinates to features, managing data sets, designing the base map, and enhancing accuracy of feature classes and CAD feature locations. This project completed the following major objectives:

- Georeference CAD files

- Design the geodatabase

- Create and design a base map

- Enhance the accuracy of some CAD feature locations

Georeferencing CAD files were the process to assign geographic coordinates to CAD files. Then necessary CAD data layers were extracted and converted to feature classes. Feature classes have the same geographic coordinates for georeferenced CAD file. Therefore, spatial data from many sources in a global coordinate system can be related to the Caltech map.

Data sets that provided by this project include feature classes and aerial photos. They were stored in one container, a file geodatabase, and organized into feature data sets and a raster catalog. The project also provided metadata about data sets. In addition, attributes were identified for feature classes. Moreover, subtypes and domains were created to provide an efficient way for editing and using less storage space in the geodatabase. Furthermore, non useful CAD properties were deleted from the table of attributes, and enclosed polygon features were provided.

A base map was created and designed for the client. The base map design includes assigning symbols, scales, and labels to display maps interactively and clear. There were aerial photos and 38 feature classes were provided to the client. Some of these feature classes were extracted from other layers or digitized.

The accuracy for some feature classes that converted from CAD files that had inaccurate locations of features was enhanced. Finally, feature classes were converted to CAD data, so the client has clean CAD data with better accuracy in their feature locations.

\subsection{Future Work}

The project established GIS for the Facilities Department at Caltech so that users in the department can use all GIS functionality and apply it for their needs. Therefore, there are many possible future works for the Facilities Department, as discussed in Chapter 2.

Three dimensional (3-D) visual could be modeled and animated for Caltech campus using ArcGIS. The 3-D visual enables users in the Facilities Department to effectively visualize, plan, analyze, and design spatial data. The 3-D visual can be created using 
ArcScene application. For example, users in the Facilities Department can create a 3-D virtual tour of Caltech. They can also view a feature from multiple viewpoints and walk around or fly through digitally. In addition, users can use the 3-D visual for analysis of evacuation routes or crime patterns on campus. This provides the best visual interpretation for planners and decision makers. According to Michael Parkin, the Facilities Department at MIT has applied GIS using 3-D analyst to visualize, analyze, and manage space at campus. Not only that but also GIS is brought inside their buildings. Spatial data infrastructure is created from their interior building spaces. This is used for space management, facilities management, public safety, and campus planning (Parkin, 2008).

The Facilities Department can also implement GIS for strategic planning, such as accessibility planning, pedestrian planning, emergency planning and response, and facility space planning. GIS can provide interactive maps to planners and students to display and illustrate handicap accessible routes and buildings, bicycle routes, and bus stops and routes. A GIS also can be applied to locate the best areas of evacuation and evacuation routes, and tell if an area of campus is well protected in the event of any emergency. Moreover, the Facilities Department can use GIS to manage classroom space, identify all features in the classroom, and show the detailed floor plans of campus buildings.

One of the best ways to communicate effectively with people is through the web. An important tool for communication is GIS for the web. Many of GIS applications can present interactive maps through the web, such as event location maps, campus base maps, and strategic planning maps. According to Miceli, the Facilities Department can also apply GIS to track work orders and show that in the web. The track work order application provides a daily list of tasks, and shows task descriptions with their locations in maps. The application would relieve maintenance crews from returning to the office during the day as new work orders come in (Miceli, 2003). 


\section{Works Cited}

Addison, A. (2006, July). Northwest Missouri State University. Retrieved from librarytheses: https://www.nwmissouri.edu/library/theses/AddisonAaron/

Bassham, C. R. (1994). GIS/MUNICIPAL MANAGEMENT INFORMATION SYSTEM AT TEXAS A\&M UNIVERSITY. Retrieved May 10, 2010, from Texas A\&M University: http://libraries.maine.edu/Spatial/gisweb/spatdb/amfm/am94056.html

Bolsted, P. (2008). GIS Fundamentals. (Third Edition). White Bear Lake: Eider Press.

ESRI. (2008). GIS desktop Help 9.3. Redlands, CA, United States of America.

Kathryn, C. (2002). Geographic Information Systems Master Plan. Retrieved June 8, 2009, from City of Salisbury: http://gis.salisburync.org/media/strategic_plan/2001_gis_master_plan.pdf

Lewis, J., \& Nell, D. B. (2007). Computer science illuminated. (Second Edition). Sudbury: Jones and Bartlett Publishers.

London, S. (2006). Mapping handicapped accessibility facilities. Retrieved April 28, 2010, from Ferris State University: http://btcsure1.ferris.edu/gisproject/

Miceli, R. (2003). Facilities Management Geographic Information System for the University of Redlands. Redlands: University of Redlands.

Parkin, M. (2008). GIS for Facilities Management. Retrieved May 10, 2010, from ESRI: http://www.esri.com/industries/facilities-management/index.html

Ramroop, S. (2006, August 7). 3-D model development case study at Troy University. Retrieved April 15, 2010, from ESRI Library: http://proceedings.esri.com/library/userconf/proc06/papers/papers/pap_1139.pdf

SBL Geomatics Group. (2009, March 10). Georeferencing and CAD GIS Services. Retrieved June 10, 2009, from Articles Base: http://www.articlesbase.com/outsourcing-articles/georeferencing-and-cad-gisservices-809905.html

Weiss, M. D. (2009, April 14). Ten Innovative Uses for GIS in Facility Management. Retrieved June 5, 2009, from advanced-infrastructure: http://www.advancedinfrastructure.com/content/ten-innovative-uses-gis-facility-management 



\section{Appendix A. Schema Report}

\section{Report Creation}

Date

Author

System Information

Operating System

.Net Framework

Diagrammer

Geodatabase

Workspace Type

File

\section{ArcGIS Diagrammer}

Saturday, February 27, 2010

Anas_Bingaith/SPATIAL on ANAS_BINGAITH

Microsoft Windows NT 5.1.2600 Service Pack 3

2.0.50727.3603

1.0.3422.27552

Personal Geodatabase

C:IMIP\Deliverable\MIP_Deliverable_Caltech_Anas\XML_DATA_SCHEMA.XML

\section{Table Of Contents}

\begin{tabular}{ll} 
Domains & Listing of Coded Value and Range Domains. \\
ObjectClasses & Listing of Tables and FeatureClasses. \\
\hline Spatial Reference & Listing of Spatial References used by FeatureClasses and FeatureDatasets.
\end{tabular}

\begin{tabular}{|c|c|c|}
\hline \multicolumn{3}{|c|}{ Domains } \\
\hline Domain Name & Owner & Domain Type \\
\hline AnnotationStatus & & Coded Value \\
\hline BooleanSymbolValue & & Coded Value \\
\hline Covered & & Coded Value \\
\hline HorizontalAlignment & & Coded Value \\
\hline Material & & Coded Value \\
\hline$\overline{\text { Type }}$ & & Coded Value \\
\hline VerticalAlignment & & Coded Value \\
\hline
\end{tabular}

\section{AnnotationStatus}

\begin{tabular}{ll}
$\begin{array}{l}\text { Owner } \\
\text { Description } \\
\text { Domain Type } \\
\text { Field Type } \\
\text { Merge Policy }\end{array}$ & $\begin{array}{l}\text { Valid annotation state values. } \\
\text { Coded Value } \\
\text { Split Policy } \\
\text { Domain Members } \\
\text { Default Value }\end{array}$ \\
$\begin{array}{l}\text { Name } \\
\text { Duplicate }\end{array}$ \\
$\begin{array}{l}\text { Placed } \\
\text { Unplaced }\end{array}$ & Value \\
$\begin{array}{l}\text { BooleanSymbolValue } \\
\text { Owner }\end{array}$ \\
$\begin{array}{l}\text { Description } \\
\text { Domain Type }\end{array}$ & 1 \\
Field Type & Valid values are Yes and No. \\
\hline & Coded Value \\
\hline
\end{tabular}




$\begin{array}{ll}\begin{array}{l}\text { Merge Policy } \\ \text { Split Policy } \\ \text { Domain Members } \\ \text { Name }\end{array} & \begin{array}{l}\text { Default Value } \\ \text { Duplicate }\end{array} \\ \text { Yes } & \text { Value } \\ \text { No } & 1 \\ \end{array}$

\section{Covered}

\section{Owner}

Description

Domain Type Coded Value

Field Type Small Integer

Merge Policy Default Value

Split Policy Default Value

Domain Members

Name

Value

Yes

0

No

HorizontalAlignment

Owner

Description

Domain Type

Field Type

Merge Policy

Split Policy

Domain Members

Name

Left

Center

Right

Full

\section{Material}

Owner

Description

Domain Type

Field Type

Merge Policy

Split Policy

Domain Members

Name

\section{Type}

Owner

Description

Domain Type

Field Type

Merge Policy

Split Policy

Domain Members

Name

Surface Lot

Multi Level

Sub-teranian
Valid horizontal symbol alignment values.

Coded Value

Small Integer

Default Value

Duplicate

\section{Value}

0

1

2

3
Coded Value

Small Integer

Default Value

Default Value

Value

Coded Value

Small Integer

Default Value

Default Value

Value

0

1

2 


\section{VerticalAlignment}

\section{Owner}

Description

Domain Type

Field Type

Merge Policy

Split Policy

Domain Members

Name

Top

Valid symbol vertical alignment values.

Coded Value

Small Integer

Default Value

Duplicate

Center

Value

Baseline

Bottom

0

1

2

3

\section{ObjectClasses}

\begin{tabular}{|c|c|c|c|c|}
\hline ObjectClass Name & $\begin{array}{c}\text { Type } \\
\end{array}$ & Geometry & Subtype & \\
\hline \multicolumn{4}{|c|}{ Ground_Facilities } & $\underline{\mathrm{SR}}$ \\
\hline Boundaries & Simple FeatureClass & Polygon & - & \\
\hline building & Simple FeatureClass & Polygon & - & \\
\hline Building_Name_anno & Annotation FeatureClass & Polygon & - & \\
\hline Building_No_anno & Annotation FeatureClass & Polygon & - & \\
\hline Emergency_Telephone & Simple FeatureClass & Point & $\begin{array}{c}\text { East } \\
\text { North } \\
\text { North East } \\
\text { North West } \\
\text { South } \\
\text { South East } \\
\text { South West } \\
\text { West }\end{array}$ & \\
\hline Fire_Hydrant & Simple FeatureClass & Point & - & \\
\hline Light_Pole & Simple FeatureClass & Point & - & \\
\hline$\underline{\text { manhole }}$ & Simple FeatureClass & Point & $\begin{array}{c}\text { Electrical Vaul } \\
\text { Sewer } \\
\text { Telecom } \\
\text { Tunnel }\end{array}$ & \\
\hline palm & Simple FeatureClass & Point & - & \\
\hline Parking_Label & Simple FeatureClass & Point & - & \\
\hline Parking_Line & Simple FeatureClass & Polyline & - & \\
\hline Parking_Lot & Simple FeatureClass & Polygon & - & \\
\hline Pool_area & Simple FeatureClass & Polygon & - & \\
\hline$\underline{\text { Sidewalk }}$ & Simple FeatureClass & Polygon & - & \\
\hline Sport_Field & Simple FeatureClass & Polygon & - & \\
\hline street & Simple FeatureClass & Polyline & - & \\
\hline street_anno & Annotation FeatureClass & Polygon & - & \\
\hline street_Polygon & Simple FeatureClass & Polygon & - & \\
\hline Tree_cover & Simple FeatureClass & Polygon & - & \\
\hline$\underline{\text { Vegetation }}$ & Simple FeatureClass & Polygon & - & \\
\hline
\end{tabular}




\begin{tabular}{|c|c|c|c|}
\hline \multicolumn{4}{|c|}{ Underground_Facilities } \\
\hline Chilled & Simple FeatureClass & Polyline & - \\
\hline chilled_water_anno & Annotation FeatureClass & Polygon & - \\
\hline compressed_air & Simple FeatureClass & Polyline & - \\
\hline compressed_air_anno & Annotation FeatureClass & Polygon & - \\
\hline condensate & Simple FeatureClass & Polyline & - \\
\hline condensate_water_anno & Annotation FeatureClass & Polygon & - \\
\hline Demonized & Simple FeatureClass & Polyline & - \\
\hline Demonized_water_anno & Annotation FeatureClass & Polygon & - \\
\hline Domestic & Simple FeatureClass & Polyline & - \\
\hline Domestic_water_anno & Annotation FeatureClass & Polygon & - \\
\hline Hot & Simple FeatureClass & Polyline & - \\
\hline hot water anno & Annotation FeatureClass & Polygon & - \\
\hline sewer & Simple FeatureClass & Polyline & - \\
\hline sewer_anno & Annotation FeatureClass & Polygon & - \\
\hline steam & Simple FeatureClass & Polyline & - \\
\hline steam_anno & Annotation FeatureClass & Polygon & - \\
\hline storm & Simple FeatureClass & Polyline & - \\
\hline$\overline{\text { tunnel }}$ & Simple FeatureClass & Polyline & - \\
\hline \multicolumn{4}{|c|}{ Stand Alone ObjectClass(s) } \\
\hline
\end{tabular}

\section{Boundaries}

\section{Alias \\ Boundaries}

Dataset Type FeatureClass

FeatureType Simple

Field Name

OBJECTID

Shape

Shape_Length

Shape_Area

Subtype Name

Index Name

FDO_OBJECTID

FDO_Shape

\begin{tabular}{l} 
Alias Name \\
OBJECTID \\
Shape \\
Shape_Length \\
Shape_Area \\
\multicolumn{1}{c}{ Default Value } \\
Ascending \\
Yes \\
No
\end{tabular}

Geometry:Polygon

Average Number of Points: 0

Has M:No

Has Z:Yes

Grid Size: 3600

Model Name Type Precn.Scale LengthNull

$\begin{array}{llllll}\text { OBJECTID OID } & 0 & 0 & 4 & \text { No }\end{array}$

Shape Geometry $0 \begin{array}{llll}0 & 0 & \text { Yes }\end{array}$

$\begin{array}{llllll}\text { Shape_Length } & \text { Double } & 0 & 0 & 8 & \text { Yes } \\ \text { Shape_Area } & \text { Double } & 0 & 0 & 8 & \text { Yes }\end{array}$

Unique Domain Fields

Yes OBJECTID

No Shape

\section{building}

\begin{tabular}{|c|c|c|}
\hline $\begin{array}{l}\text { Alias } \\
\text { Dataset Type }\end{array}$ & $\begin{array}{l}\text { building } \\
\text { FeatureClass }\end{array}$ & \\
\hline FeatureType & Simple & \\
\hline $\begin{array}{l}\text { Field Name } \\
\text { OBJECTID } \\
\text { Shape } \\
\text { Shape_Length } \\
\text { Shape_Area } \\
\text { Name } \\
\text { Number } \\
\text { Type } \\
\text { Street } \\
\text { Building_Condition } \\
\text { Type_of_construction } \\
\text { Landmark_Status }\end{array}$ & & $\begin{array}{l}\text { Alias Name } \\
\text { OBJECTID } \\
\text { Shape } \\
\text { Shape_Length } \\
\text { Shape_AArea }\end{array}$ \\
\hline Year_of_construction & & \\
\hline Number_of_floors & & \\
\hline
\end{tabular}

Geometry:Polygon

Average Number of Points:0

Has M:No

Has Z:Yes

Grid Size: 240

Model Name Type Precn.Scale LengthNull OBJECTID

Shape

Shape_Length

Shape_Area 


\begin{tabular}{llll} 
Subtype Name & \multicolumn{1}{c}{ Default Value } & Domain \\
Index Name & Ascending & Unique & Fields \\
FDO_OBJECTID & Yes & Yes & OBJECTID \\
FDO_Shape & No & No & Shape
\end{tabular}

\section{Building_Name_anno}

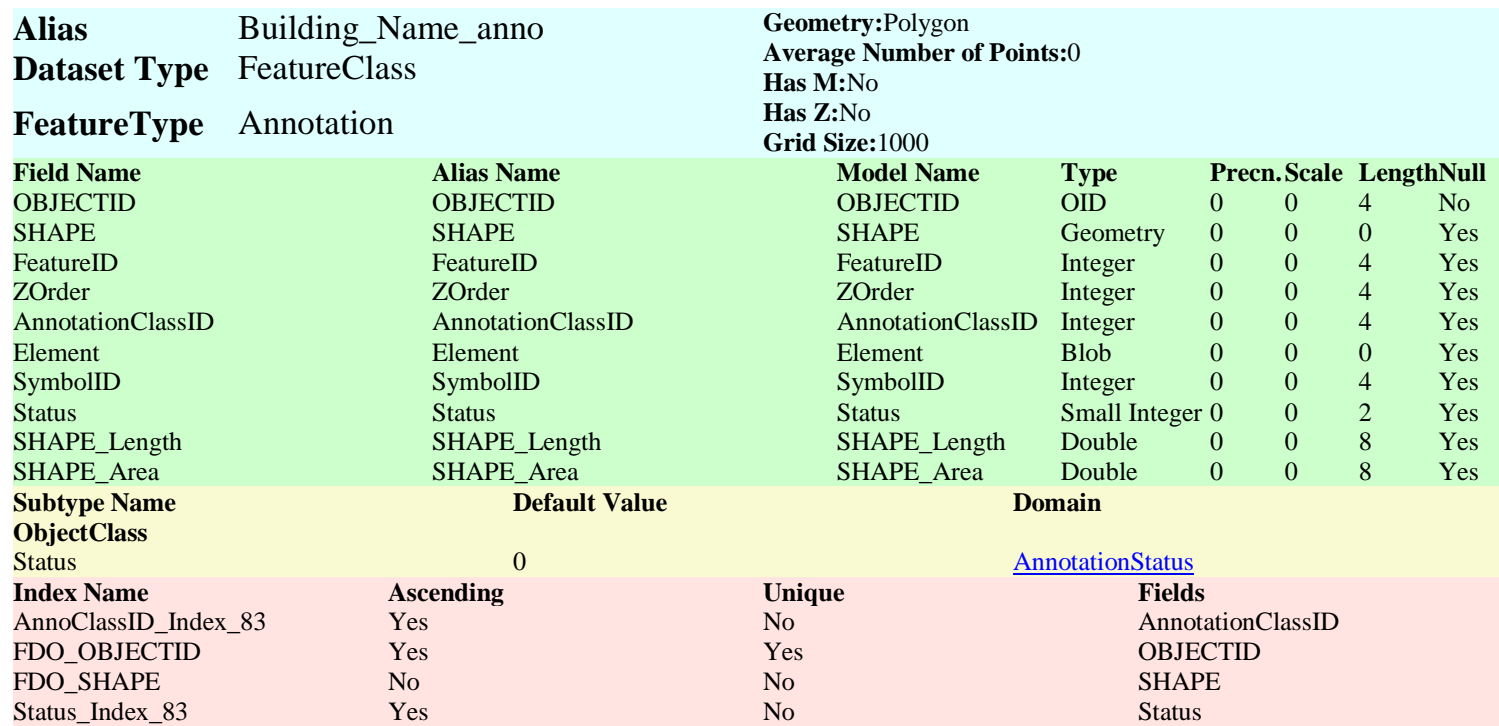

\section{Building_No_anno}

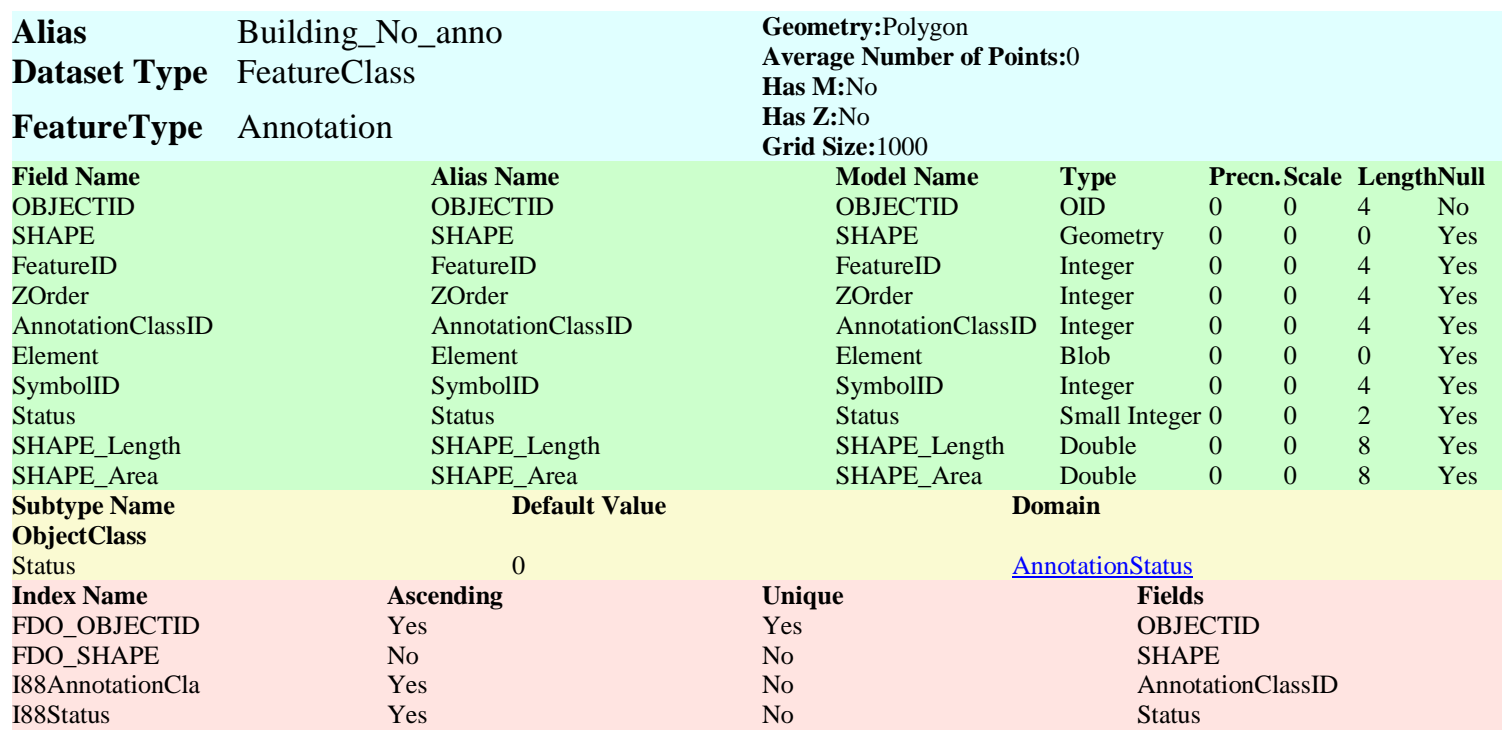

\section{Chilled}

\section{Alias \\ Dataset Type FeatureClass \\ FeatureType Simple \\ Chilled}

Field Name

OBJECTID

Shape

Shape_Length

Install_Date

Size

Source

Supply_Return
Alias Name

OBJECTID

Shape

Shape_Length
Geometry:Polyline

Average Number of Points:0

Has M:No

Has Z:Yes

Grid Size: 320

\begin{tabular}{llllll} 
Model Name & Type & \multicolumn{2}{l}{ Precn. Scale } & LengthNull \\
OBJECTID & OID & 0 & 0 & 4 & No \\
Shape & Geometry & 0 & 0 & 0 & Yes \\
Shape_Length & Double & 0 & 0 & 8 & Yes \\
& Date & 0 & 0 & 8 & Yes \\
& Single & 0 & 0 & 4 & Yes \\
& String & 0 & 0 & 15 & Yes \\
& String & 0 & 0 & 15 & Yes
\end{tabular}




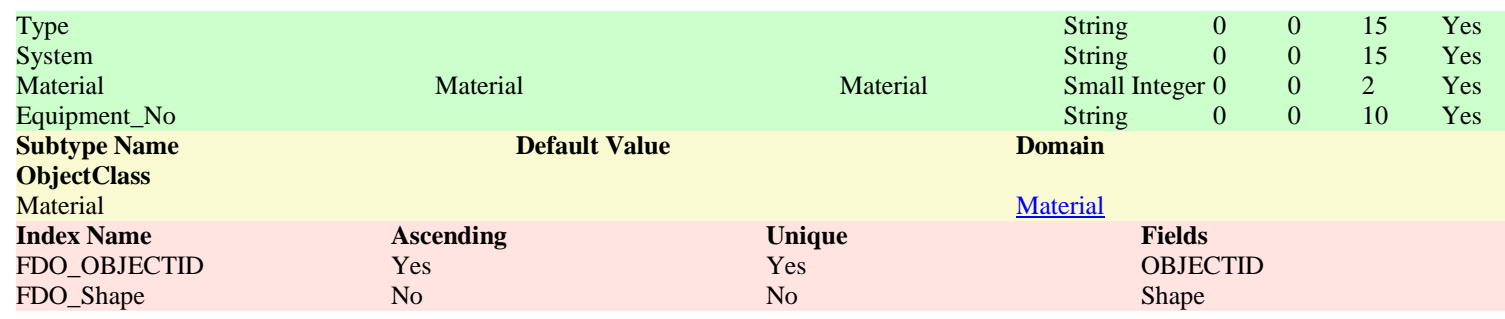

chilled_water_anno

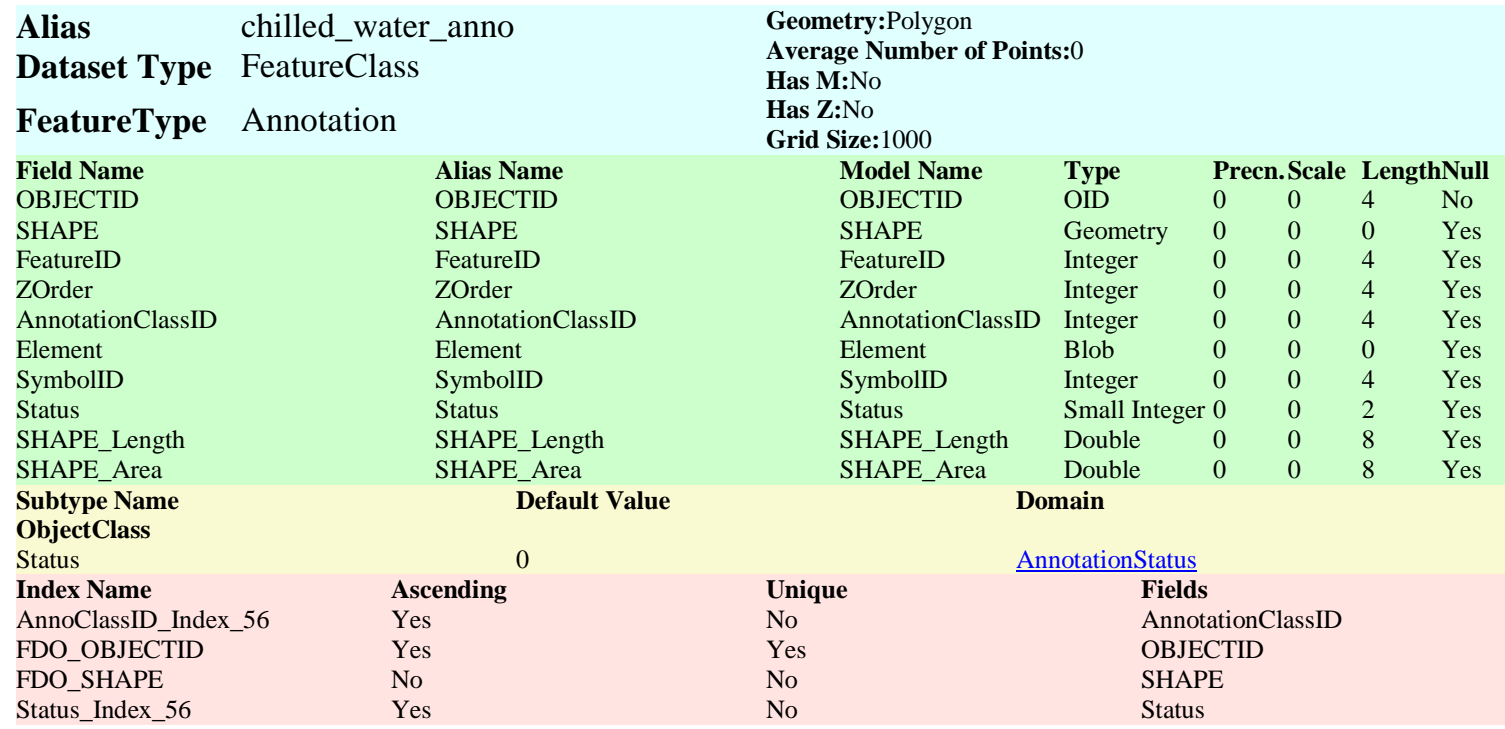

\section{compressed_air}

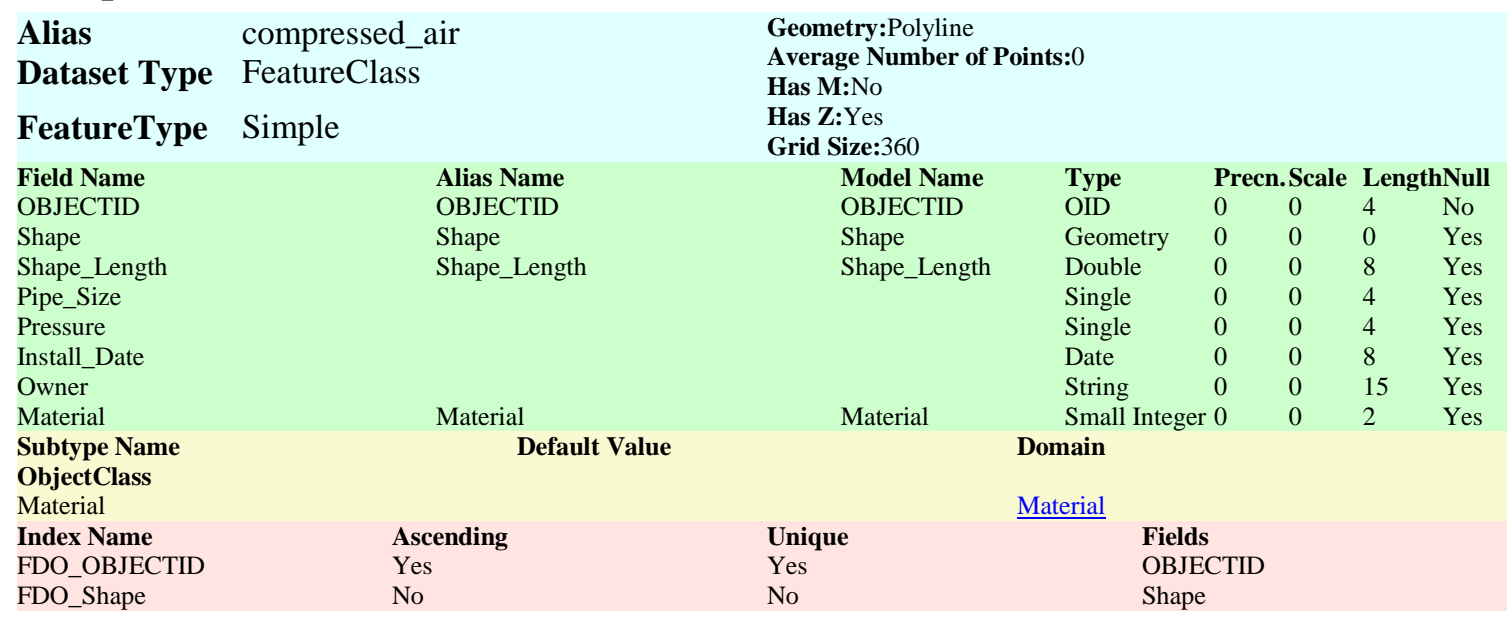

\section{compressed_air_anno}

Alias compressed_air_anno

Dataset Type FeatureClass

FeatureType Annotation

Field Name

OBJECTID

SHAPE

FeatureID

ZOrder

AnnotationClassID
Alias Name

OBJECTID

SHAPE

FeatureID

ZOrder

AnnotationClassID
Geometry:Polygon

Average Number of Points:0

Has M:No

Has Z:No

Grid Size: 1000

\begin{tabular}{llllll} 
Model Name & Type & \multicolumn{3}{c}{ Precn. Scale } & LengthNull \\
OBJECTID & OID & 0 & 0 & 4 & No \\
SHAPE & Geometry & 0 & 0 & 0 & Yes \\
FeatureID & Integer & 0 & 0 & 4 & Yes \\
ZOrder & Integer & 0 & 0 & 4 & Yes \\
AnnotationClassID & Integer & 0 & 0 & 4 & Yes
\end{tabular}




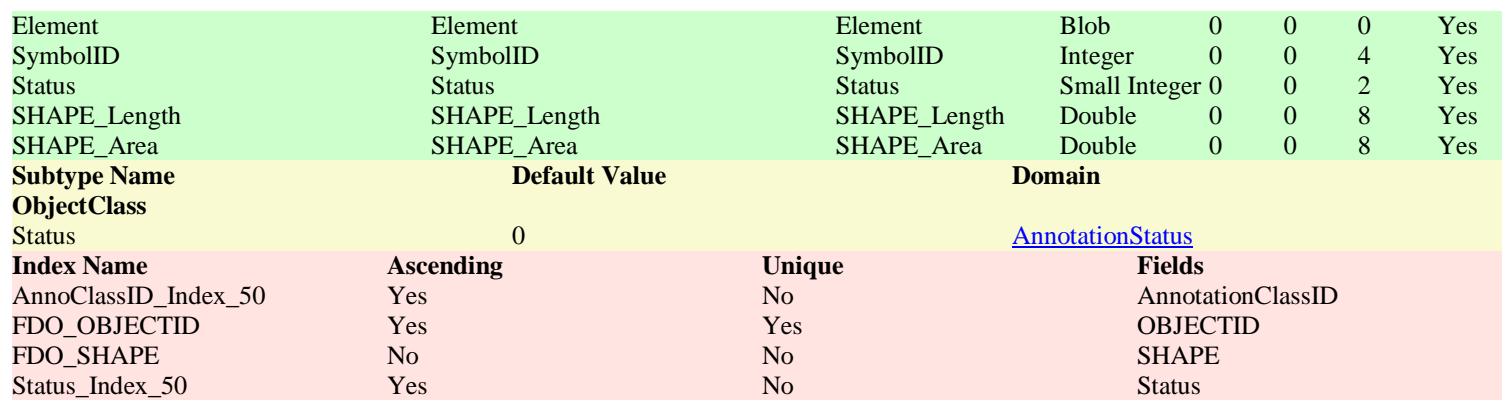

\section{condensate}

\begin{tabular}{|c|c|c|c|c|c|c|c|}
\hline $\begin{array}{l}\text { Alias } \\
\text { Dataset Type }\end{array}$ & $\begin{array}{l}\text { condensate } \\
\text { FeatureClass }\end{array}$ & \multicolumn{6}{|c|}{$\begin{array}{l}\text { Geometry:Polyline } \\
\text { Average Number of Points:0 }\end{array}$} \\
\hline FeatureType & Simple & $\begin{array}{l}\text { Has Z:Yes } \\
\text { Grid Size: } 1100\end{array}$ & & & & & \\
\hline Field Name & Alias Name & Model Name & Type & Precr & Scale & Len & hNull \\
\hline OBJECTID & OBJECTID & OBJECTID & OID & 0 & 0 & 4 & No \\
\hline Shape & Shape & Shape & Geometry & 0 & 0 & 0 & Yes \\
\hline Shape_Length & Shape_Length & Shape_Length & Double & 0 & 0 & 8 & Yes \\
\hline Equip_No & & & String & 0 & 0 & 15 & Yes \\
\hline Install_Date & & & Date & 0 & 0 & 8 & Yes \\
\hline Size & & & Single & 0 & 0 & 4 & Yes \\
\hline Source & & & String & 0 & 0 & 15 & Yes \\
\hline Supply_return & & & String & 0 & 0 & 15 & Yes \\
\hline Type & & & String & 0 & 0 & 15 & Yes \\
\hline System & & & String & 0 & 0 & 15 & Yes \\
\hline Material & Material & Material & Small Integer & & 0 & 2 & Yes \\
\hline $\begin{array}{l}\text { Subtype Name } \\
\text { ObjectClass }\end{array}$ & Default Value & & nain & & & & \\
\hline Material & & & erial & & & & \\
\hline Index Name & Ascending & Unique & Fields & & & & \\
\hline FDO_OBJECTID & Yes & Yes & OBJEC & CTID & & & \\
\hline FDO_Shape & No & No & Shape & & & & \\
\hline
\end{tabular}

\section{condensate_water_anno}

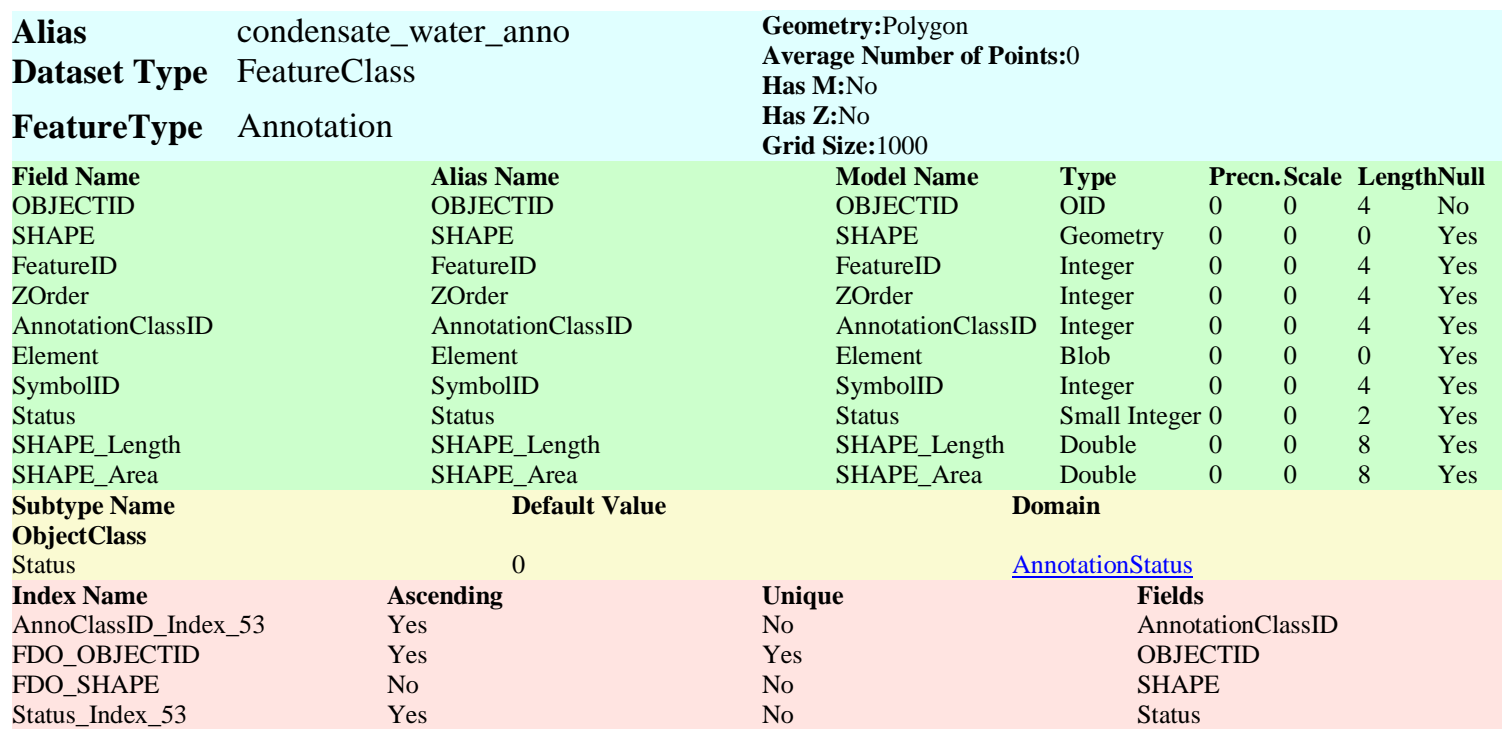

\section{Demonized}
Alias
Demonized
Dataset Type FeatureClass
Geometry:Polyline
Average Number of Points:0
FeatureType Simple

\author{
Has M:No \\ Has Z:Yes \\ Grid Size: 570
}




\begin{tabular}{|c|c|c|c|c|c|c|c|}
\hline Field Name & \multirow{2}{*}{$\begin{array}{l}\text { Alias Name } \\
\text { OBJECTID }\end{array}$} & \multirow{2}{*}{$\begin{array}{l}\text { Model Name } \\
\text { OBJECTID }\end{array}$} & Type & \multicolumn{2}{|c|}{ Precn.Scale } & \multicolumn{2}{|c|}{ LengthNull } \\
\hline OBJECTID & & & OID & 0 & 0 & 4 & No \\
\hline Shape & Shape & Shape & Geometry & 0 & 0 & 0 & Yes \\
\hline Shape_Length & Shape_Length & Shape_Length & Double & 0 & 0 & 8 & Yes \\
\hline Equip_No & & & String & 0 & 0 & 10 & Yes \\
\hline Install_Date & & & Date & 0 & 0 & 8 & Yes \\
\hline Size & & & Single & 0 & 0 & 4 & Yes \\
\hline Source & & & String & 0 & 0 & 15 & Yes \\
\hline Supply_Return & & & String & 0 & 0 & 15 & Yes \\
\hline Type & & & String & 0 & 0 & 15 & Yes \\
\hline System & & & String & 0 & 0 & 15 & Yes \\
\hline Material & Material & Material & \multicolumn{2}{|c|}{ Small Integer 0} & 0 & 2 & Yes \\
\hline Subtype Name & \multicolumn{2}{|c|}{ Default Value } & \multirow{2}{*}{\multicolumn{5}{|c|}{ Domain }} \\
\hline \multicolumn{3}{|l|}{ ObjectClass } & & & & & \\
\hline Material & & & \multicolumn{5}{|c|}{ Iaterial } \\
\hline Index Name & \multicolumn{2}{|l|}{ Ascending } & \multicolumn{3}{|c|}{ Fields } & & \\
\hline FDO_OBJECTID & Yes & Yes & \multicolumn{3}{|c|}{ OBJECTID } & & \\
\hline FDO_Shape & No & No & \multicolumn{3}{|c|}{ Shape } & & \\
\hline
\end{tabular}

\section{Demonized_water_anno}

Alias Demonized_water_anno Geometry:Polygon

Dataset Type FeatureClass

FeatureType Annotation

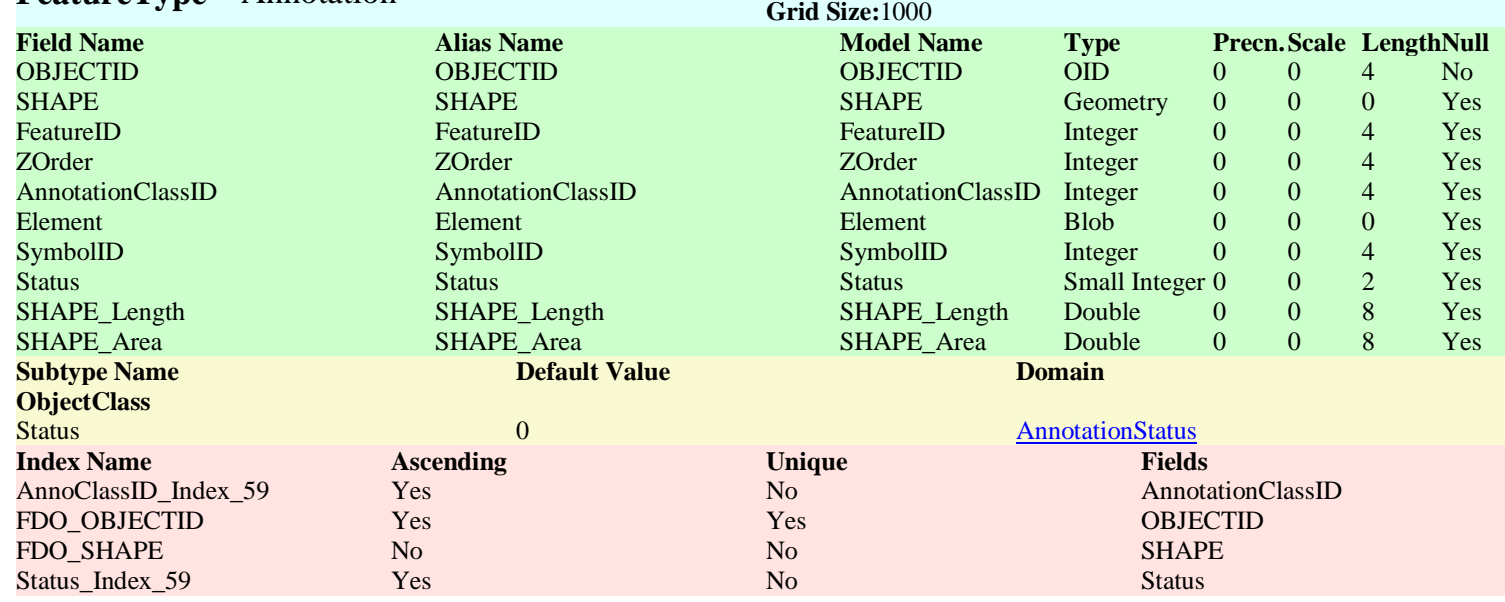

\section{Domestic}

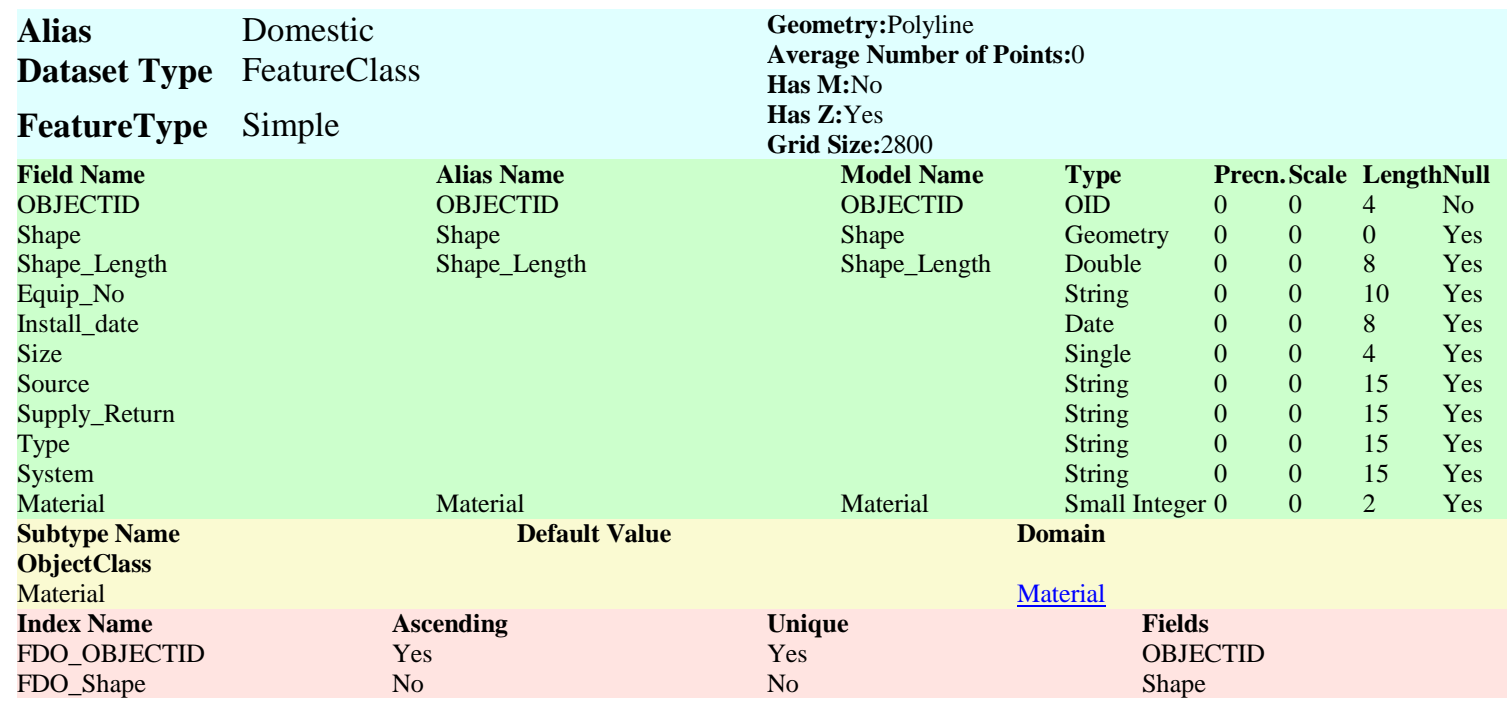

\section{Domestic_water_anno}




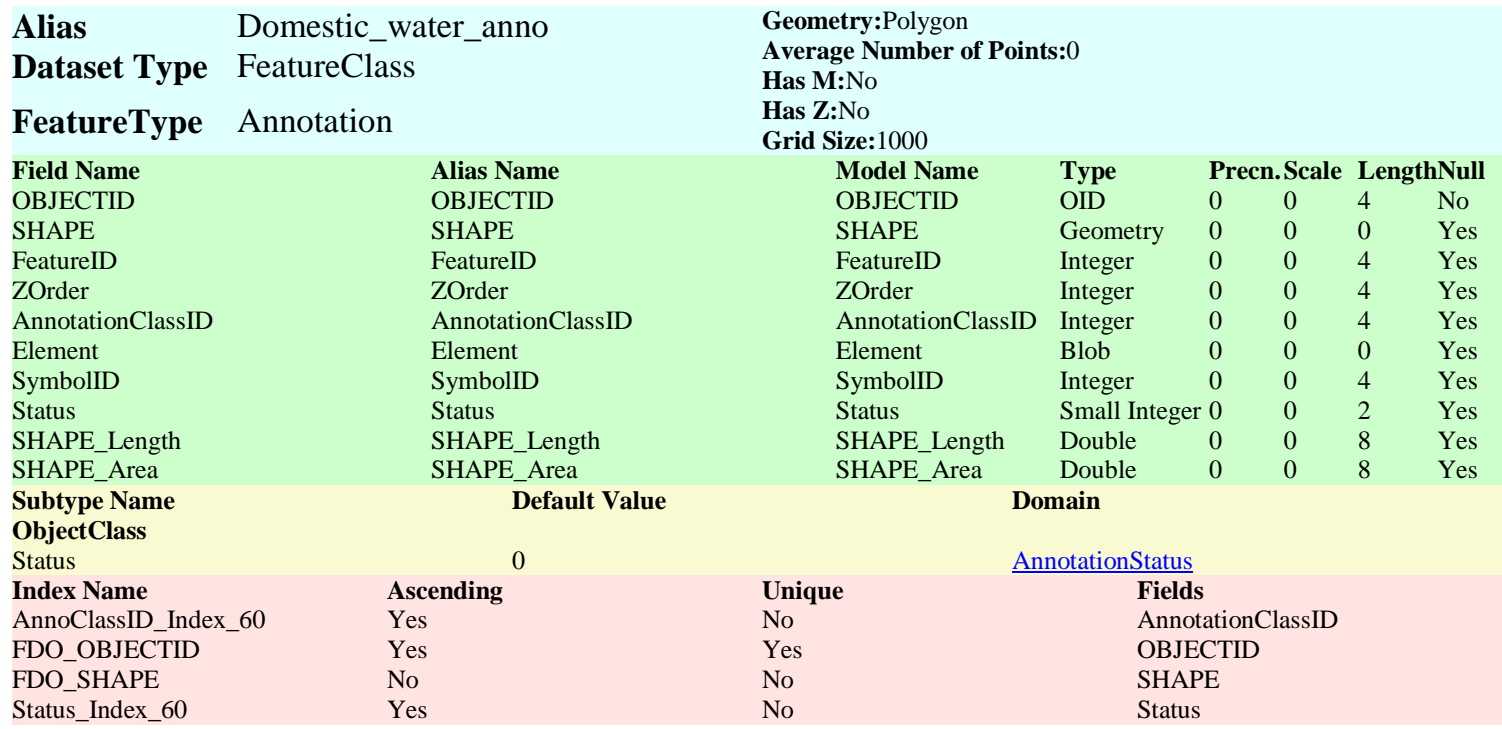

\section{Emergency_Telephone}

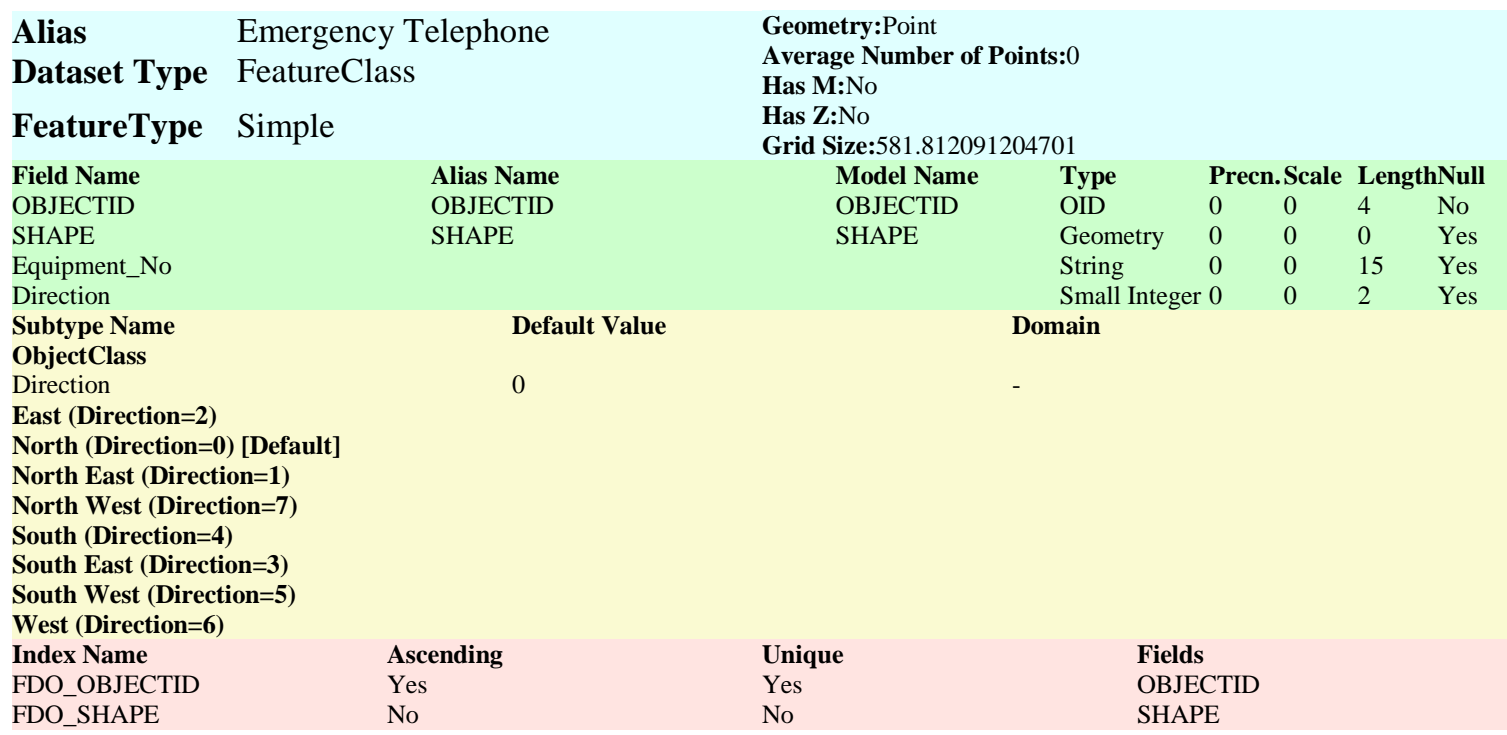

\section{Fire_Hydrant}

Alias Fire_Hydrant

Dataset Type FeatureClass

FeatureType Simple

$\begin{array}{ll}\text { Field Name } & \text { A } \\ \text { OBJECTID } & \text { OBJ } \\ \text { Shape } & \\ \text { Elevation } & \\ \text { Style } & \text { Mount } \\ \text { Pressure } & \\ \text { Flow } & \text { Update_Test_date } \\ \text { Owner }\end{array}$

Owner

Subtype Name

Index Name

FDO_OBJECTID

FDO_Shape
Geometry:Point

Average Number of Points:0

Has M:No

Has Z:Yes

Grid Size:493.523966334221

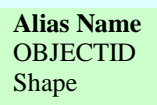

Model Name
OBJECTID
Shape

Type
OID
Geometry

Double

String

String

Single

Single

Date

String

Default Value

Domain

Unique

Yes

Fields

OBJECTID

Shape

\begin{tabular}{llll}
\multicolumn{3}{l}{ Precn. Scale } & \multicolumn{2}{l}{ LengthNull } \\
0 & 0 & 4 & No \\
0 & 0 & 0 & Yes \\
0 & 0 & 8 & No \\
0 & 0 & 15 & Yes \\
0 & 0 & 15 & Yes \\
0 & 0 & 4 & Yes \\
0 & 0 & 4 & Yes \\
0 & 0 & 8 & Yes \\
0 & 0 & 15 & Yes \\
& & & \\
CTID & & &
\end{tabular}


Hot

\begin{tabular}{|c|c|c|c|c|c|c|c|}
\hline \multirow{3}{*}{$\begin{array}{l}\text { Alias } \\
\text { Dataset Type } \\
\text { FeatureType }\end{array}$} & \multirow{2}{*}{$\begin{array}{l}\text { Hot } \\
\text { FeatureClass }\end{array}$} & \multicolumn{6}{|c|}{ Geometry:Polyline } \\
\hline & & \multicolumn{6}{|c|}{$\begin{array}{l}\text { Geometry:Polyline } \\
\text { Average Number of Points:0 } \\
\text { Has M:No }\end{array}$} \\
\hline & Simple & $\begin{array}{l}\text { Has Z:Yes } \\
\text { Grid Size: } 380\end{array}$ & & & & & \\
\hline Field Name & Alias Name & Model Name & Type & Precr & Scale & LeI & hNull \\
\hline OBJECTID & OBJECTID & OBJECTID & OID & 0 & 0 & 4 & No \\
\hline Shape & Shape & Shape & Geometry & 0 & 0 & 0 & Yes \\
\hline Shape_Length & Shape_Length & Shape_Length & Double & 0 & 0 & 8 & Yes \\
\hline Equip_No & & & String & 0 & 0 & 15 & Yes \\
\hline Install_date & & & Date & 0 & 0 & 8 & Yes \\
\hline Size & & & Single & 0 & 0 & 4 & Yes \\
\hline Source & & & String & 0 & 0 & 15 & Yes \\
\hline Supply_Return & & & String & 0 & 0 & 15 & Yes \\
\hline Type & & & String & 0 & 0 & 15 & Yes \\
\hline System & & & String & 0 & 0 & 15 & Yes \\
\hline Material & Material & Material & Small Integer & 0 & 0 & 2 & Yes \\
\hline $\begin{array}{l}\text { Subtype Name } \\
\text { ObjectClass }\end{array}$ & Default Value & & main & & & & \\
\hline Material & & & terial & & & & \\
\hline Index Name & Ascending & Unique & Fields & & & & \\
\hline FDO_OBJECTID & Yes & Yes & OBJEC & CTID & & & \\
\hline FDO_Shape & No & No & Shape & & & & \\
\hline
\end{tabular}

\section{hot_water_anno}

\section{Alias \\ hot_water_anno \\ Dataset Type FeatureClass}

FeatureType Annotation

Field Name

OBJECTID

SHAPE

FeatureID

ZOrder

AnnotationClassID

Element

SymbolID

Status

SHAPE_Length

SHAPE_Area

Subtype Name

ObjectClass

Status

Index Name

AnnoClassID_Index_58

FDO_OBJECTID

FDO_SHAPE

Status_Index_58

\begin{tabular}{l} 
Alias Name \\
OBJECTID \\
SHAPE \\
FeatureID \\
ZOrder \\
AnnotationClassID \\
Element \\
SymbolID \\
Status \\
SHAPE_Length \\
SHAPE_Area \\
\multicolumn{1}{c}{ Default Value } \\
$\quad 0$ \\
scending \\
Ses
\end{tabular}

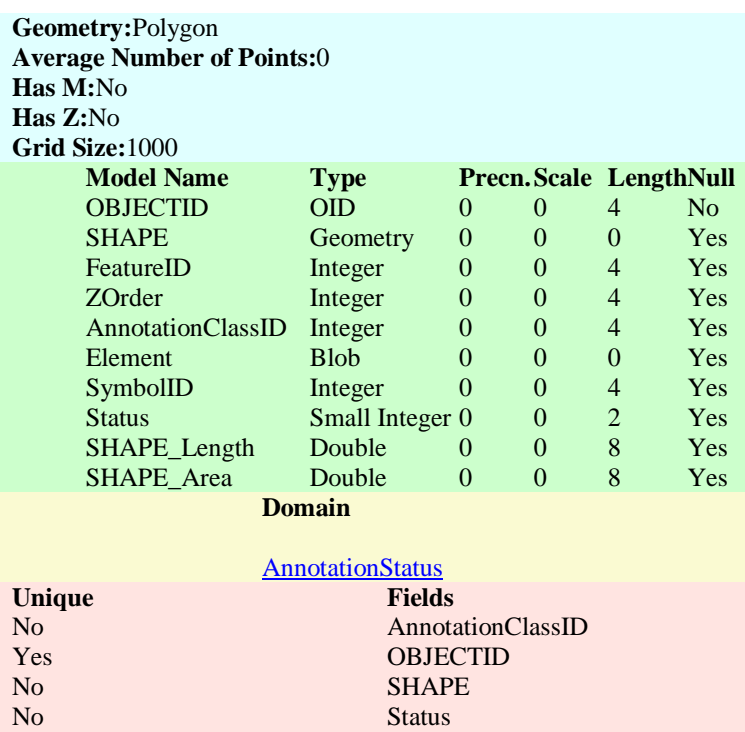

\section{Light_Pole}

\section{Alias \\ Light_Pole \\ Dataset Type FeatureClass \\ FeatureType Simple}

\begin{tabular}{ll}
$\begin{array}{l}\text { Field Name } \\
\text { OBJECTID }\end{array}$ & \multicolumn{1}{c}{$\begin{array}{c}\text { Alias Name } \\
\text { OBJECTID } \\
\text { Shape }\end{array}$} \\
Lamp_Type & Shape \\
Manufacturer & \\
Pole_Height & \\
Equipment_No & \\
Voltage & \\
Walttage & \\
Ref_Name & \\
Subtype Name & \\
Index Name & \\
FDO_OBJECTID & Ascending Default Value \\
FDO_Shape & Yes
\end{tabular}

\section{OBJECTID}

Shape

Pole_Height

Equipment_No

Walttag

Ref_Name

Index Name

FDO_Shape
Geometry:Point

Average Number of Points:0

Has M:No

Has Z:Yes

Grid Size:133.294906068946

\begin{tabular}{llllll} 
Model Name & Type & \multicolumn{3}{l}{ Precn. Scale } & LengthNull \\
OBJECTID & OID & 0 & 0 & 4 & No \\
Shape & Geometry & 0 & 0 & 0 & Yes \\
& String & 0 & 0 & 10 & Yes \\
& String & 0 & 0 & 10 & Yes \\
& Single & 0 & 0 & 4 & Yes \\
& String & 0 & 0 & 15 & Yes \\
& Small Integer 0 & 0 & 2 & Yes \\
& Small Integer 0 & 0 & 2 & Yes \\
& String & 0 & 0 & 10 & Yes \\
& Domain & & & \\
& \multicolumn{3}{c}{ Fields } \\
& OBJECTID & & \\
Shape &
\end{tabular}


manhole

$\begin{array}{ll}\text { Alias } & \text { manhole } \\ \text { Dataset Type } & \text { FeatureClass } \\ \text { FeatureType } & \text { Simple }\end{array}$

$\begin{array}{ll}\text { Field Name } & \text { Alias Name } \\ \text { OBJECTID } & \text { OBJECTID } \\ \text { Shape } & \text { Shape } \\ \text { Elevation } & \\ \text { Cavity_Length } & \text { Cavity_Size } \\ \text { Cavity_Width } & \\ \text { Cavity_Depth } & \\ \text { Cover_Diameter } & \\ \text { Type } & \end{array}$

Subtype Name

ObjectClass

Type

Electrical Vault $($ Type $=1$ )

Sewer $($ Type $=0)$ [Default]

Telecom (Type $=3$ )

Tunnel $($ Type $=2)$

Index Name

FDO_OBJECTID

FDO Shape
Default Value

0
Geometry:Point

Average Number of Points:0

Has M:No

Has Z:Yes

Grid Size:241.115101184432

Model Name

OBJECTID

Shape

Type

Geometry

Double

Cavity_Length

Single

Single

Single

Single

Small Integer 0

Domain

\begin{tabular}{cccc} 
Precn. Scale & \multicolumn{3}{l}{ LengthNull } \\
0 & 0 & 4 & No \\
0 & 0 & 0 & Yes \\
0 & 0 & 8 & No \\
0 & 0 & 4 & Yes \\
0 & 0 & 4 & Yes \\
0 & 0 & 4 & Yes \\
0 & 0 & 4 & Yes \\
0 & 0 & 2 & Yes
\end{tabular}

\section{palm}

Alias

Dataset Type FeatureClass

FeatureType Simple

Field Name
OBJECTID
Shape
Trunk_Size
Height
Subtype Name
Index Name
FDO_OBJECTID
FDO_Shape

palm

\section{Ascending}

Yes

No

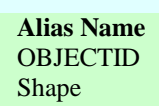

Default Value

Default Value
Ascending
Yes
No

Geometry:Point

Average Number of Points:0

Has M:Yes

Has Z:Yes

Grid Size:171.599472840054

\begin{tabular}{llllll} 
Model Name & Type & \multicolumn{3}{c}{ Precn. Scale } & LengthNull \\
OBJECTID & OID & 0 & 0 & 4 & No \\
Shape & Geometry & 0 & 0 & 0 & Yes \\
& $\begin{array}{l}\text { Single } \\
\text { Single }\end{array}$ & 0 & 0 & 4 & Yes \\
& Domain & 0 & 4 & Yes \\
& \multicolumn{3}{c}{ Fields } \\
OBJECTID & & \\
Shape &
\end{tabular}

\section{Parking_Label}

\section{Alias \\ Parking Label \\ Dataset Type FeatureClass \\ FeatureType Simple}

Field Name
OBJECTID
SHAPE
Subtype Name
Index Name
FDO_OBJECTID
FDO_SHAPE

\section{Parking_Line}

Alias

Dataset Type FeatureClass

FeatureType Simple

Field Name

OBJECTID

Shape

Shape_Length

Subtype Name

Index Name

FDO_OBJECTID

FDO_Shape
Parking_Line

Alias Name

OBJECTID

SHAPE

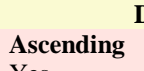

Yes

No

\section{Default Value}

C Yes

Fields

OBJECTID

Shape 


\section{Parking_Lot}

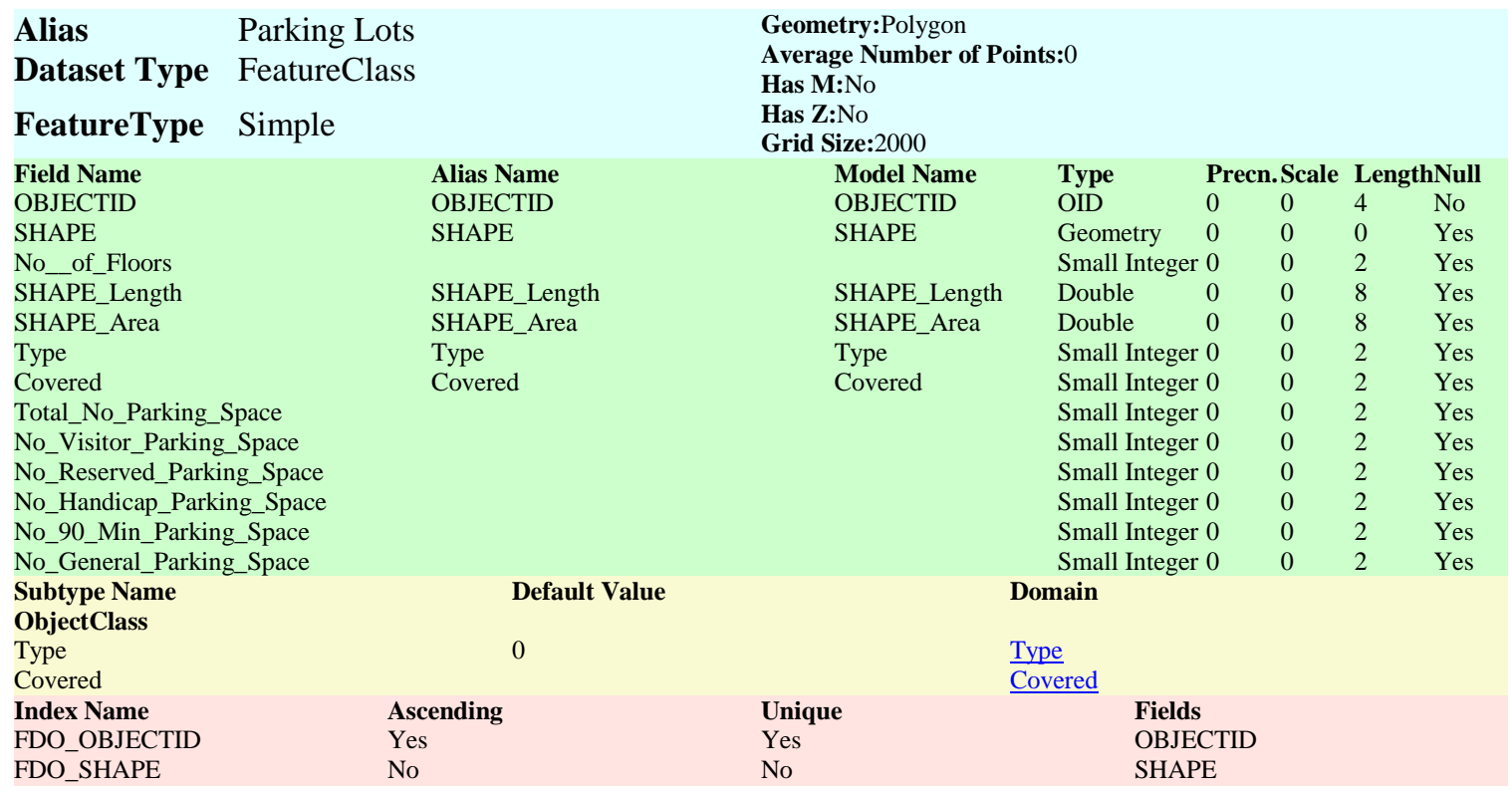

\section{Pool_area}

\section{Alias \\ Dataset Type FeatureClass \\ FeatureType Simple}

$\begin{array}{ll}\text { Field Name } & \text { Alias Name } \\ \text { OBJECTID } & \text { OBJECTID } \\ \text { Shape } & \text { Shape } \\ \text { Shape_Length } & \text { Shape_Length } \\ \text { Shape_Area } & \text { Shape_Area } \\ \text { Dimensions } & \\ \text { Type } & \end{array}$

Name

Subtype Name

Index Name

FDO_OBJECTID

FDO_Shape
Default Value

$\begin{array}{ll}\text { Ascending } & \text { Unique } \\ \text { Yes } & \text { Yes } \\ \text { No } & \text { No }\end{array}$

Geometry:Polygon

Average Number of Points:0

Has M:No

Has Z:Yes

Grid Size: 310

\begin{tabular}{llllll} 
Model Name & Type & \multicolumn{3}{c}{ Precn.Scale } & LengthNull \\
OBJECTID & OID & 0 & 0 & 4 & No \\
Shape & Geometry & 0 & 0 & 0 & Yes \\
Shape_Length & Double & 0 & 0 & 8 & Yes \\
Shape_Area & Double & 0 & 0 & 8 & Yes \\
& Single & 0 & 0 & 4 & Yes \\
& String & 0 & 0 & 15 & Yes \\
& String & 0 & 0 & 10 & Yes \\
& Domain & & & \\
& Fields \\
OBJECTID & & \\
& Shape
\end{tabular}

\section{sewer}

\begin{tabular}{|c|c|c|c|c|c|c|c|}
\hline \multirow{2}{*}{$\begin{array}{l}\text { Alias } \\
\text { Dataset Type } \\
\text { FeatureType }\end{array}$} & $\begin{array}{l}\text { sewer } \\
\text { FeatureClass }\end{array}$ & \multicolumn{6}{|c|}{$\begin{array}{l}\text { Geometry:Polyline } \\
\text { Average Number of Points:0 }\end{array}$} \\
\hline & Simple & \multicolumn{6}{|c|}{$\begin{array}{l}\text { Has Z:Yes } \\
\text { Grid Size: } 730\end{array}$} \\
\hline Field Name & Alias Name & Model Name & Type & Pre & Scale & Len & hNull \\
\hline OBJECTID & OBJECTID & OBJECTID & OID & 0 & 0 & 4 & No \\
\hline Shape & Shape & Shape & Geometry & 0 & 0 & 0 & Yes \\
\hline Shape_Length & Shape_Length & Shape_Length & Double & 0 & 0 & 8 & Yes \\
\hline Size & & & Single & 0 & 0 & 4 & Yes \\
\hline Slope & & & Single & 0 & 0 & 4 & Yes \\
\hline Status & & & String & 0 & 0 & 15 & Yes \\
\hline Installation_Date & installation data & Installation_Date & Date & 0 & 0 & 8 & Yes \\
\hline Rim_Elevation & & & Single & 0 & 0 & 4 & Yes \\
\hline Invert_Elevation & & & Single & 0 & 0 & 4 & Yes \\
\hline Owner & & & String & 0 & 0 & 15 & Yes \\
\hline Subtype Name & Default Value & & nain & & & & \\
\hline Index Name & Ascending & Unique & & & & & \\
\hline FDO_OBJECTID & Yes & Yes & & CTI & & & \\
\hline FDO_Shape & No & No & Sh & & & & \\
\hline
\end{tabular}


sewer_anno

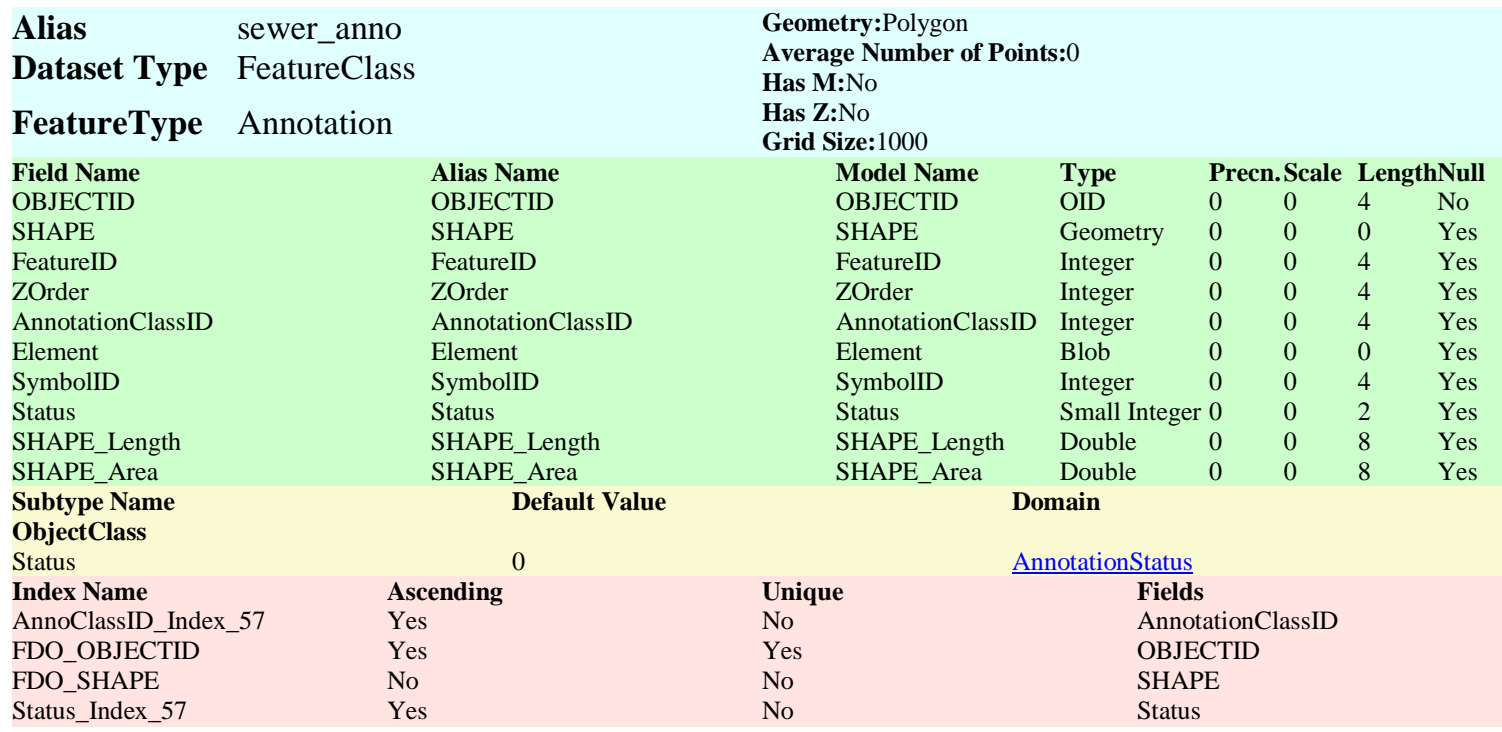

\section{Sidewalk}

\section{Alias Sidewalk \\ Dataset Type FeatureClass \\ FeatureType Simple}

Field Name

OBJECTID

Shape

Shape_Length

Shape_Area

Subtype Name

Index Name

FDO_OBJECTID

FDO_Shape

\section{Sport_Field}

$\begin{array}{ll}\text { Alias } & \text { Sport_Field } \\ \text { Dataset Type } & \text { FeatureClass } \\ \text { FeatureType } & \text { Simple }\end{array}$

\begin{tabular}{ll} 
Field Name & \multicolumn{1}{c}{ Alias Name } \\
OBJECTID & OBJECTID \\
Shape & Shape \\
Shape_Length & Shape_Length \\
Shape_Area & Shape_Area \\
Floor_Type & \multicolumn{1}{c}{ Default Value } \\
Subtype Name & \\
Index Name & Ascending \\
FDO_OBJECTID & Yes \\
FDO_Shape & No
\end{tabular}

Alias Name

OBJECTID

Shape

Shape_Length

Shape_Area

Default Value

\section{Ascending}

Yes

No
Geometry:Polygon

Average Number of Points:0

Has M:No

Has Z:Yes

Grid Size:2000

\begin{tabular}{llllll} 
Model Name & Type & \multicolumn{2}{l}{ Precn. Scale } & \multicolumn{2}{l}{ LengthNull } \\
OBJECTID & OID & 0 & 0 & 4 & No \\
Shape & Geometry & 0 & 0 & 0 & Yes \\
Shape_Length & Double & 0 & 0 & 8 & Yes \\
Shape_Area & Double & 0 & 0 & 8 & Yes
\end{tabular}

Unique Domain Fields

Yes OBJECTID

No Shape

Shape

\section{steam}

\section{Alias \\ Dataset Type FeatureClass \\ FeatureType Simple \\ steam}

Field Name

OBJECTID

Shape

Shape_Length

Equip_No
Alias Name

OBJECTID

Shape

Shape_Length
Geometry:Polygon

Average Number of Points:0

Has M:No

Has Z:Yes

Grid Size:530

\begin{tabular}{|c|c|c|c|c|c|}
\hline Model Name & Type & \multicolumn{2}{|c|}{ Precn.Scale } & \multicolumn{2}{|c|}{ LengthNull } \\
\hline OBJECTID & OID & 0 & 0 & 4 & No \\
\hline Shape & Geometry & 0 & 0 & 0 & Yes \\
\hline Shape_Length & Double & 0 & 0 & 8 & Yes \\
\hline Shape_Area & Double & 0 & 0 & 8 & Yes \\
\hline & String & 0 & 0 & 10 & Yes \\
\hline \multicolumn{6}{|c|}{ Domain } \\
\hline & $\begin{array}{l}\text { Fie } \\
\text { OB } \\
\text { Sh }\end{array}$ & & & & \\
\hline
\end{tabular}

Geometry:Polyline

Average Number of Points:0

Has M:No

Has Z:Yes

Grid Size: 440

\begin{tabular}{llllll} 
Model Name & Type & \multicolumn{3}{c}{ Precn.Scale } & \multicolumn{2}{l}{ LengthNull } \\
OBJECTID & OID & 0 & 0 & 4 & No \\
Shape & Geometry & 0 & 0 & 0 & Yes \\
Shape_Length & Double & 0 & 0 & 8 & Yes \\
& String & 0 & 0 & 10 & Yes
\end{tabular}




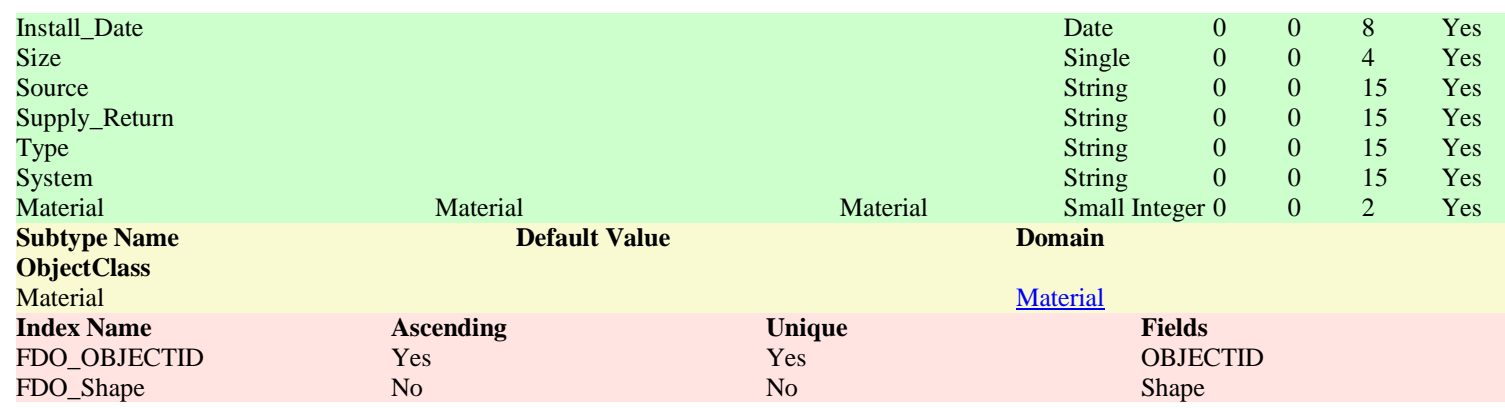

\section{steam_anno}

Alias steam_anno

Dataset Type FeatureClass

FeatureType Annotation

\section{Field Name}

OBJECTID

SHAPE

FeatureID

ZOrder

AnnotationClassID

Element

SymbolID

Status

SHAPE_Length

SHAPE_Area

Subtype Name

ObjectClass

Status

Index Name

AnnoClassID_Index_55

FDO_OBJECTID

FDO_SHAPE

Status_Index_55

Alias Name
OBJECTID
SHAPE
FeatureID
ZOrder
AnnotationClassID
Element
SymbolID
Status
SHAPE_Length
SHAPE_Area $\quad$ Default Value

Ascending
Yes
Yes
No
Yes

Geometry:Polygon

Average Number of Points:0

Has M:No

Has Z:No

Grid Size: 1000

\begin{tabular}{llllll} 
Model Name & Type & \multicolumn{3}{l}{ Precn. Scale } & LengthNull \\
OBJECTID & OID & 0 & 0 & 4 & No \\
SHAPE & Geometry & 0 & 0 & 0 & Yes \\
FeatureID & Integer & 0 & 0 & 4 & Yes \\
ZOrder & Integer & 0 & 0 & 4 & Yes \\
AnnotationClassID & Integer & 0 & 0 & 4 & Yes \\
Element & Blob & 0 & 0 & 0 & Yes \\
SymbolID & Integer & 0 & 0 & 4 & Yes \\
Status & Small Integer 0 & 0 & 2 & Yes \\
SHAPE_Length & Double & 0 & 0 & 8 & Yes \\
SHAPE_Area & Double & 0 & 0 & 8 & Yes \\
& Domain & & & &
\end{tabular}

AnnotationStatus

Unique Fields

No AnnotationClassID

Yes OBJECTID

No SHAPE

No Status

\section{storm}

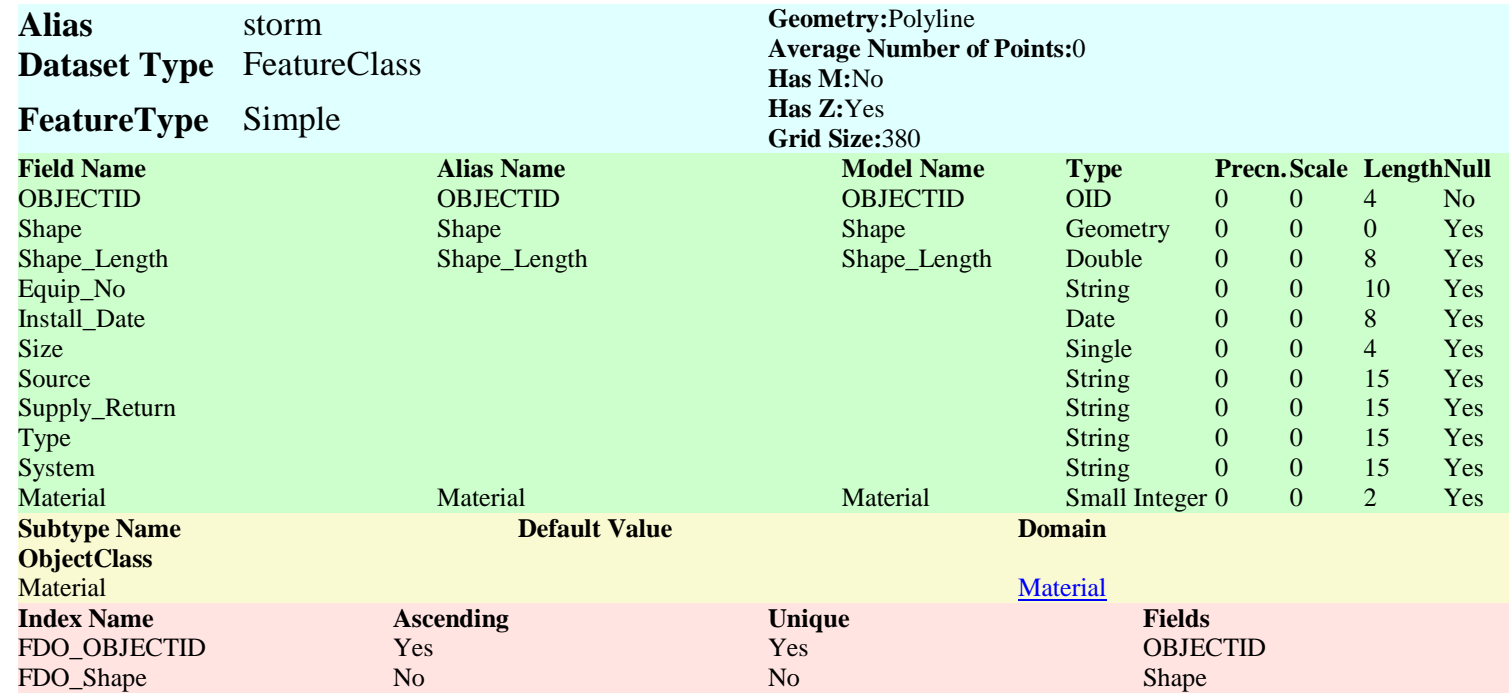




\begin{tabular}{|c|c|c|c|c|c|c|c|}
\hline $\begin{array}{l}\text { Alias } \\
\text { Dataset Type }\end{array}$ & $\begin{array}{l}\text { street } \\
\text { FeatureClass }\end{array}$ & \multicolumn{6}{|c|}{$\begin{array}{l}\text { Geometry:Polyline } \\
\text { Average Number of Points: } 0\end{array}$} \\
\hline FeatureType & Simple & $\begin{array}{l}\text { Has Z:No } \\
\text { Grid Size: } 1200\end{array}$ & & & & & \\
\hline Field Name & Alias Name & Model Name & Type & & Scale & Len & hNull \\
\hline OBJECTID & OBJECTID & OBJECTID & OID & 0 & 0 & 4 & No \\
\hline Shape & Shape & Shape & Geometry & 0 & 0 & 0 & Yes \\
\hline L_F_ADD & & & Integer & 0 & 0 & 4 & Yes \\
\hline L_T_ADD & & & Integer & 0 & 0 & 4 & Yes \\
\hline R_F_ADD & & & Integer & 0 & 0 & 4 & Yes \\
\hline R_T_ADD & & & Integer & 0 & 0 & 4 & Yes \\
\hline PREFIX & & & String & 0 & 0 & 2 & Yes \\
\hline NAME & & & String & 0 & 0 & 40 & Yes \\
\hline TYPE & & & String & 0 & 0 & 20 & Yes \\
\hline FCC & & & String & 0 & 0 & 3 & Yes \\
\hline ACC & & & String & 0 & 0 & 1 & Yes \\
\hline SPEED & & & Integer & 0 & 0 & 4 & Yes \\
\hline POSTAL_L & & & String & 0 & 0 & 5 & Yes \\
\hline POSTAL_R & & & String & 0 & 0 & 5 & Yes \\
\hline GEONAME_L & & & String & 0 & 0 & 40 & Yes \\
\hline GEONAME_R & & & String & 0 & 0 & 40 & Yes \\
\hline STATE_L & & & String & 0 & 0 & 2 & Yes \\
\hline STATE_R & & & String & 0 & 0 & 10 & Yes \\
\hline STATE & & & String & 0 & 0 & 40 & Yes \\
\hline COUNTRY & & & String & 0 & 0 & 3 & Yes \\
\hline Label & & & String & 0 & 0 & 25 & Yes \\
\hline Shape_Length & Shape_Length & Shape_Length & Double & 0 & 0 & 8 & Yes \\
\hline Subtype Name & Default Value & & nain & & & & \\
\hline Index Name & Ascending & Unique & & & & & \\
\hline FDO_OBJECTID & Yes & Yes & $\mathrm{OB}$ & CT & & & \\
\hline FDO_Shape & No & No & Sha & & & & \\
\hline
\end{tabular}

\section{street_anno}

\section{Alias \\ Dataset Type FeatureClass}

\section{FeatureType Annotation}

\begin{tabular}{|c|}
\hline Field Name \\
\hline OBJECTID \\
\hline SHAPE \\
\hline FeatureID \\
\hline ZOrder \\
\hline AnnotationClassID \\
\hline Element \\
\hline SymbolID \\
\hline Status \\
\hline SHAPE_Length \\
\hline SHAPE_Area \\
\hline Subtype Name \\
\hline ObjectClass \\
\hline Status \\
\hline Index Name \\
\hline AnnoClassID_Index_82 \\
\hline FDO_OBJECTID \\
\hline FDO_SHAPE \\
\hline Status_Index_82 \\
\hline
\end{tabular}

\begin{tabular}{l} 
Alias Name \\
OBJECTID \\
SHAPE \\
FeatureID \\
ZOrder \\
AnnotationClassID \\
Element \\
SymbolID \\
Status \\
SHAPE_Length \\
SHAPE_Area \\
\multicolumn{1}{c}{ Default Value }
\end{tabular}

Ascending
Yes
Yes
No
Yes

Geometry:Polygon

Average Number of Points:0

Has M:No

Has Z:No

Grid Size: 1000

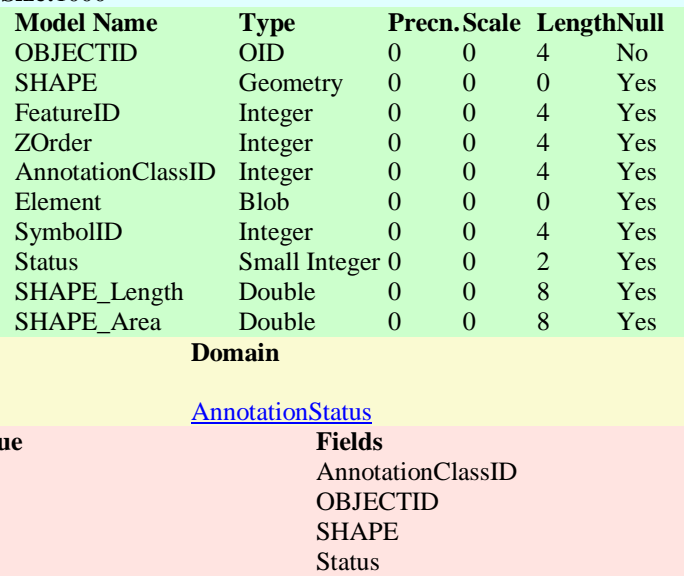

\section{street_Polygon}

Alias street_Polygon

Dataset Type FeatureClass

FeatureType Simple

$\begin{array}{ll}\text { Field Name } & \text { Alias Name } \\ \text { OBJECTID } & \text { OBJECTID } \\ \text { Shape } & \text { Shape } \\ \text { Shape_Length } & \text { Shape_Length } \\ \text { Shape_Area } & \text { Shape_Area }\end{array}$

Geometry:Polygon

Average Number of Points:0

Has M:No

Has Z:Yes

Grid Size: 3600

\begin{tabular}{llllll} 
Model Name & Type & \multicolumn{3}{c}{ Precn.Scale } & \multicolumn{2}{c}{ LengthNull } \\
OBJECTID & OID & 0 & 0 & 4 & No \\
Shape & Geometry & 0 & 0 & 0 & Yes \\
Shape_Length & Double & 0 & 0 & 8 & Yes \\
Shape_Area & Double & 0 & 0 & 8 & Yes
\end{tabular}




\begin{tabular}{llll} 
Subtype Name & \multicolumn{1}{c}{ Default Value } & Domain \\
Index Name & Ascending & Unique & Fields \\
FDO_OBJECTID & Yes & Yes & OBJECTID \\
FDO_Shape & No & No & Shape
\end{tabular}

\section{Tree cover}

$\begin{array}{ll}\text { Alias } & \text { Tree_cover } \\ \text { Dataset Type } & \text { FeatureClass }\end{array}$

FeatureType Simple

\section{Field Name}

OBJECTID

Shape

Type

Trunk_Size

Canopy_Size

Shape_Length

Shape_Area

Subtype Name

Index Name

FDO_OBJECTID

FDO_Shape
Alias Name

OBJECTID

Shape

Shape_Length Shape_Area

Ascending

Yes

No

Default Value

\section{tunnel}

\section{Alias tunnel \\ Dataset Type FeatureClass}

FeatureType Simple

Field Name
OBJECTID
Shape
Shape_Length
Size
Material
Subtype Name
ObjectClass
Material
Index Name
FDO_OBJECTID
FDO_Shape

\section{Vegetation}

$\begin{array}{ll}\text { Alias } & \text { Vegetation } \\ \text { Dataset Type } & \text { FeatureClass } \\ \text { FeatureType } & \text { Simple }\end{array}$

\section{Field Name}

OBJECTID

Shape

Type

Trunk_Size

Shape_Length

Shape_Area

Subtype Name

Index Name

FDO_OBJECTID

FDO_Shape

\section{Alias Name}

OBJECTID

Shape

Shape_Length

Material

Default Value

Ascending

Yes

No
Geometry:Polygon

Average Number of Points:0

Has M:No

Has Z:Yes

Grid Size:220

\begin{tabular}{llllll} 
Model Name & Type & \multicolumn{3}{c}{ Precn.Scale } & LengthNull \\
OBJECTID & OID & 0 & 0 & 4 & No \\
Shape & Geometry & 0 & 0 & 0 & Yes \\
& String & 0 & 0 & 15 & Yes \\
& Single & 0 & 0 & 4 & Yes \\
Shape_Length & Single & 0 & 0 & 4 & Yes \\
Shape_Area & Double & 0 & 0 & 8 & Yes \\
& Double & 0 & 0 & 8 & Yes \\
& Domain & & & \\
& Fields \\
OBJECTID & & \\
Shape
\end{tabular}

Shape
Geometry:Polyline

Average Number of Points:0

Has M:No

Has Z:Yes

Grid Size:680

Model Name Type Precn.Scale LengthNull

OBJECTID

Shape

Shape_Length

$\begin{array}{lllll}\text { OID } & 0 & 0 & 4 & \text { No } \\ \text { Geometry } & 0 & 0 & 0 & \text { Yes }\end{array}$

$\begin{array}{lllll}\text { Double } & 0 & 0 & 8 & \text { Yes }\end{array}$

Single $0=0$

Material

Small Integer 0

Domain

$\underline{\text { Material }}$

Unique $\quad$ Fields

Yes OBJECTID

No Shape
Geometry:Polygon

Average Number of Points:0

Has M:No

Has Z:Yes

Grid Size:330

\begin{tabular}{llllll} 
Model Name & Type & \multicolumn{3}{l}{ Precn.Scale } & LengthNull \\
OBJECTID & OID & 0 & 0 & 4 & No \\
Shape & Geometry & 0 & 0 & 0 & Yes \\
& String & 0 & 0 & 15 & Yes \\
Shape_Length & $\begin{array}{l}\text { Single } \\
\text { Double }\end{array}$ & 0 & 0 & 4 & Yes \\
Shape_Area & $\begin{array}{c}\text { Double } \\
\text { Domain }\end{array}$ & 0 & 0 & 8 & Yes \\
& \multicolumn{3}{c}{ Fields } \\
OBJECTID \\
Shape
\end{tabular}




\section{Spatial References}

\begin{tabular}{|c|c|c|}
\hline Dimension & Minimum & Precision \\
\hline \multicolumn{3}{|c|}{ Ground_Facilities } \\
\hline $\mathbf{X}$ & -117608900 & \multirow{2}{*}{3048.00609601219} \\
\hline $\mathbf{Y}$ & -91881400 & \\
\hline $\mathbf{M}$ & -100000 & 10000 \\
\hline $\mathbf{Z}$ & -3173.47238669141 & 1048576000488.28 \\
\hline \multicolumn{3}{|c|}{$\begin{array}{l}\text { Coordinate System Description } \\
\text { PROJCS["NAD_1983_StatePlane_California_V_FIPS_0405_Feet",GEOGCS["GCS_North_American_19 } \\
\text { 83",DATUM["D_North_American_1983",SPHEROID["GRS_1980",6378137.0,298.257222101]],PRIME } \\
\text { M["Greenwich",0.0],UNIT["Degree",0.0174532925199433]],PROJECTION["Lambert_Conformal_Conic" } \\
\text { ],PARAMETER["False_Easting",6561666.666666666],PARAMETER["False_Northing",1640416.666666 } \\
\text { 667],PARAMETER["Central_Meridian",,- } \\
\text { 118.0],PARAMETER["Standard_Parallel_1",34.03333333333333],PARAMETER["Standard_Parallel_2", } \\
\text { 35.46666666666667],PARAMETER["Latitude_Of_Origin",33.5],UNIT["Foot_US",0.3048006096012192] } \\
\text {,AUTHORITY["EPSG",2229]] }\end{array}$} \\
\hline \multicolumn{3}{|c|}{ Underground_Facilities } \\
\hline $\mathbf{X}$ & -117608900 & \multirow{2}{*}{3048.00609601219} \\
\hline $\mathbf{Y}$ & -91881400 & \\
\hline $\mathbf{M}$ & -100000 & 10000 \\
\hline $\mathrm{Z}$ & -100000 & 10000 \\
\hline \multicolumn{3}{|c|}{$\begin{array}{l}\text { Coordinate System Description } \\
\text { PROJCS["NAD_1983_StatePlane_California_V_FIPS_0405_Feet",GEOGCS["GCS_North_American_19 } \\
\text { 83",DATUM["D_North_American_1983",SPHEROID["GRS_1980",6378137.0,298.257222101]],PRIME } \\
\text { M["Greenwich",0.0],UNIT["Degree",0.0174532925199433]],PROJECTION["Lambert_Conformal_Conic" } \\
\text { ],PARAMETER["False_Easting",6561666.666666666],PARAMETER["False_Northing",1640416.666666 } \\
\text { 667],PARAMETER["Central_Meridian",- } \\
\text { 118.0],PARAMETER["Standard_Parallel_1",34.033333333333333],PARAMETER["Standard_Parallel_2", } \\
\text { 35.46666666666667],PARAMETER["Latitude_Of_Origin",33.5],UNIT["Foot_US",0.3048006096012192] } \\
\text {,AUTHORITY["EPSG",2229]] }\end{array}$} \\
\hline
\end{tabular}





\section{Appendix B. Ground Facility Campus Maps}

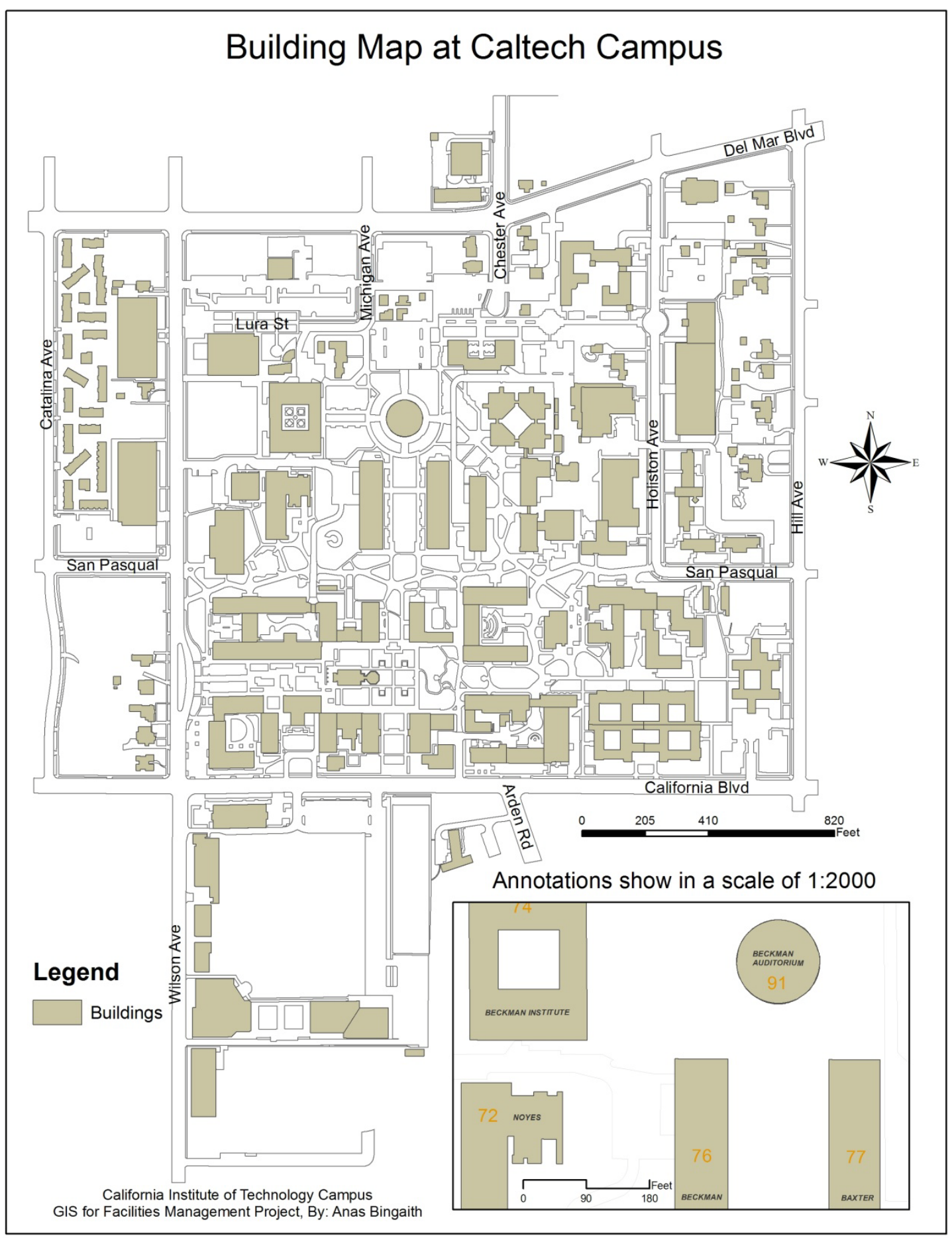




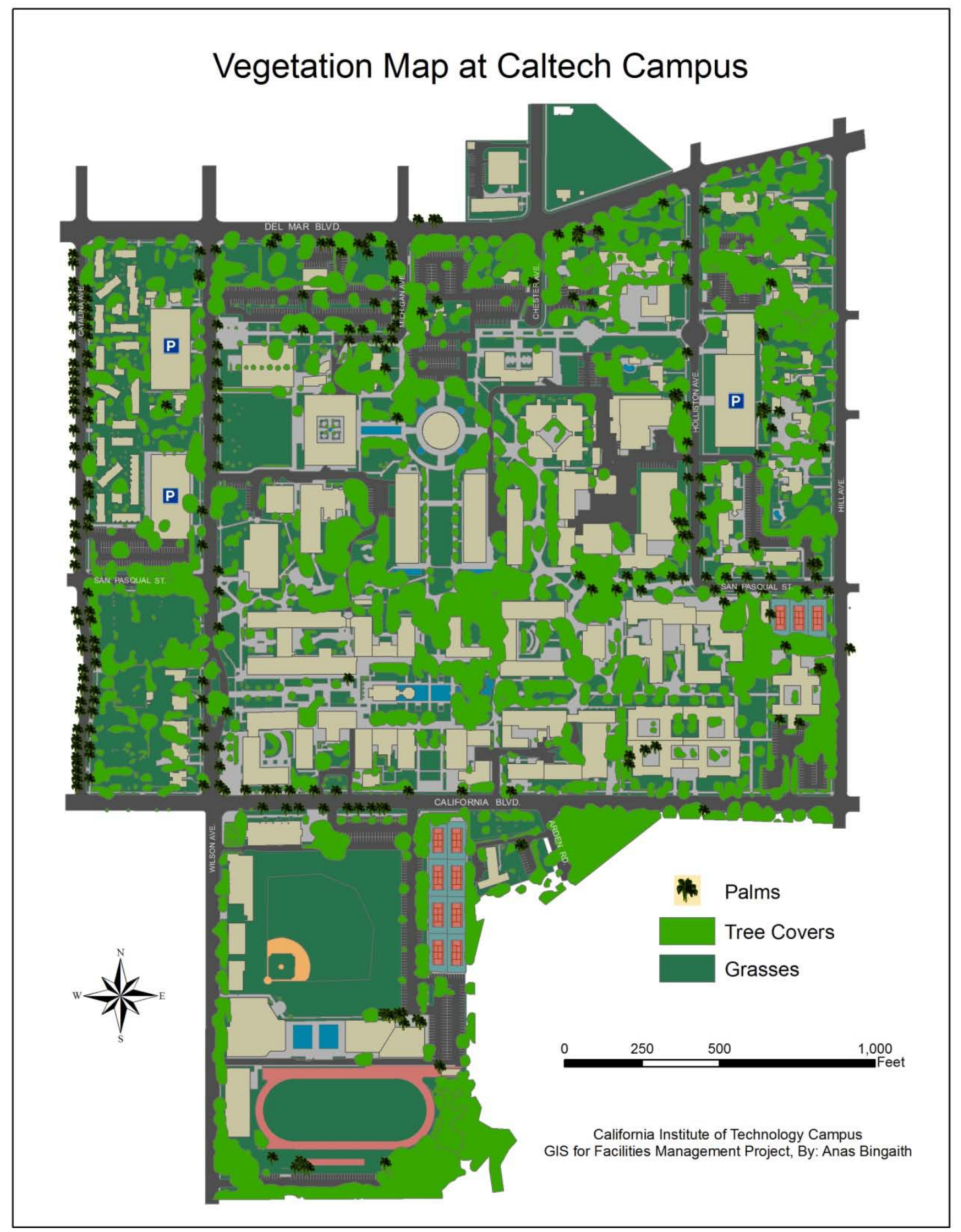




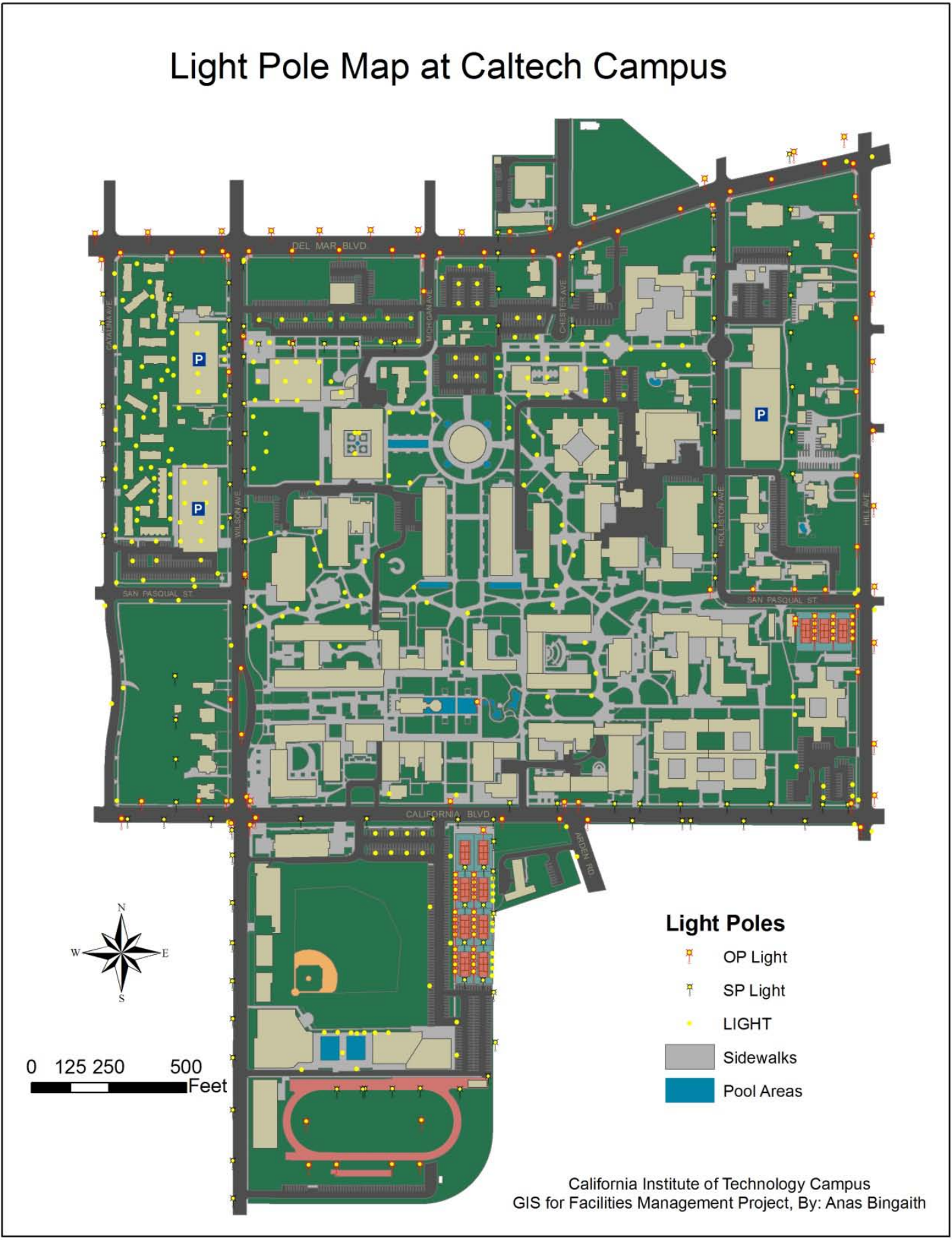




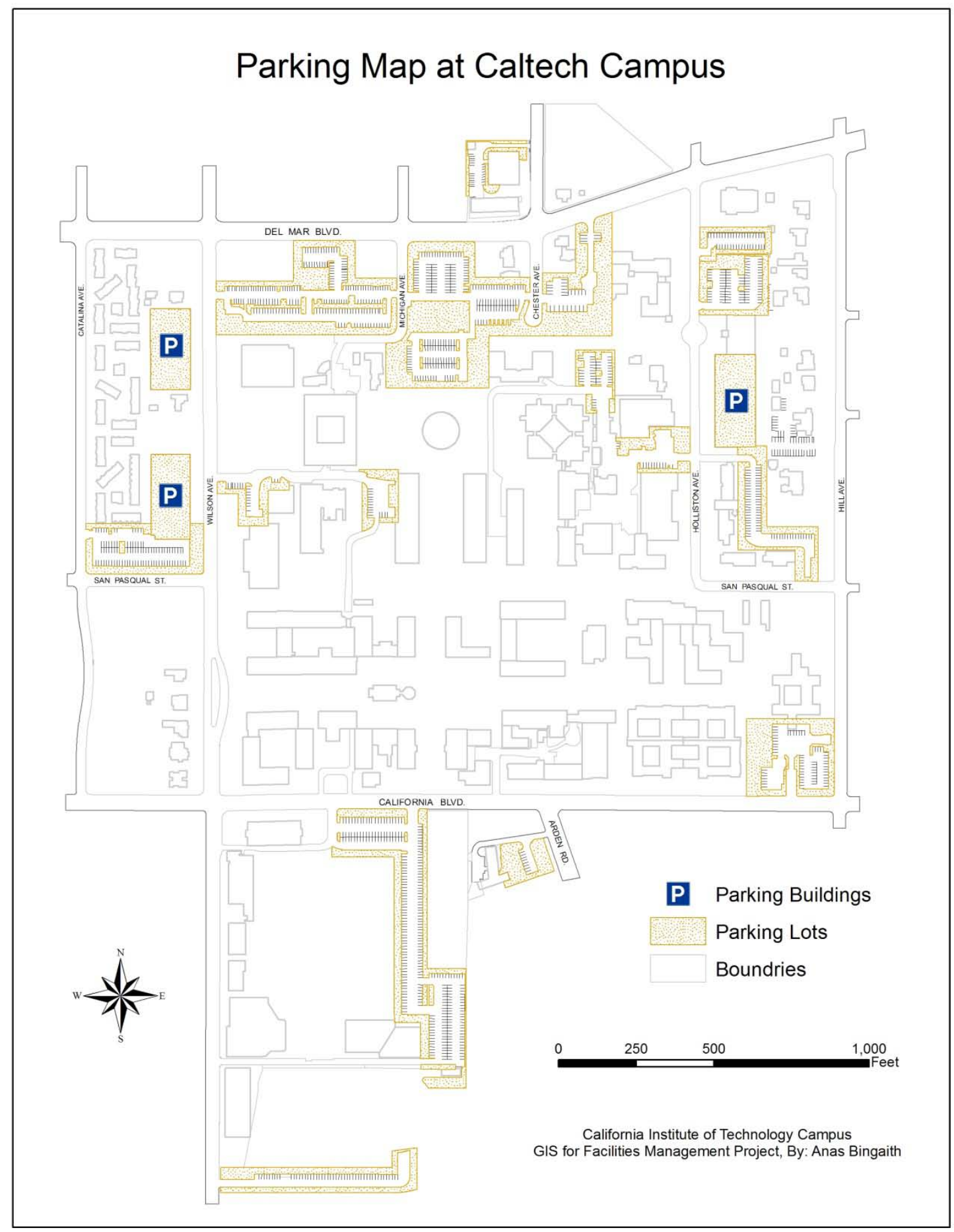




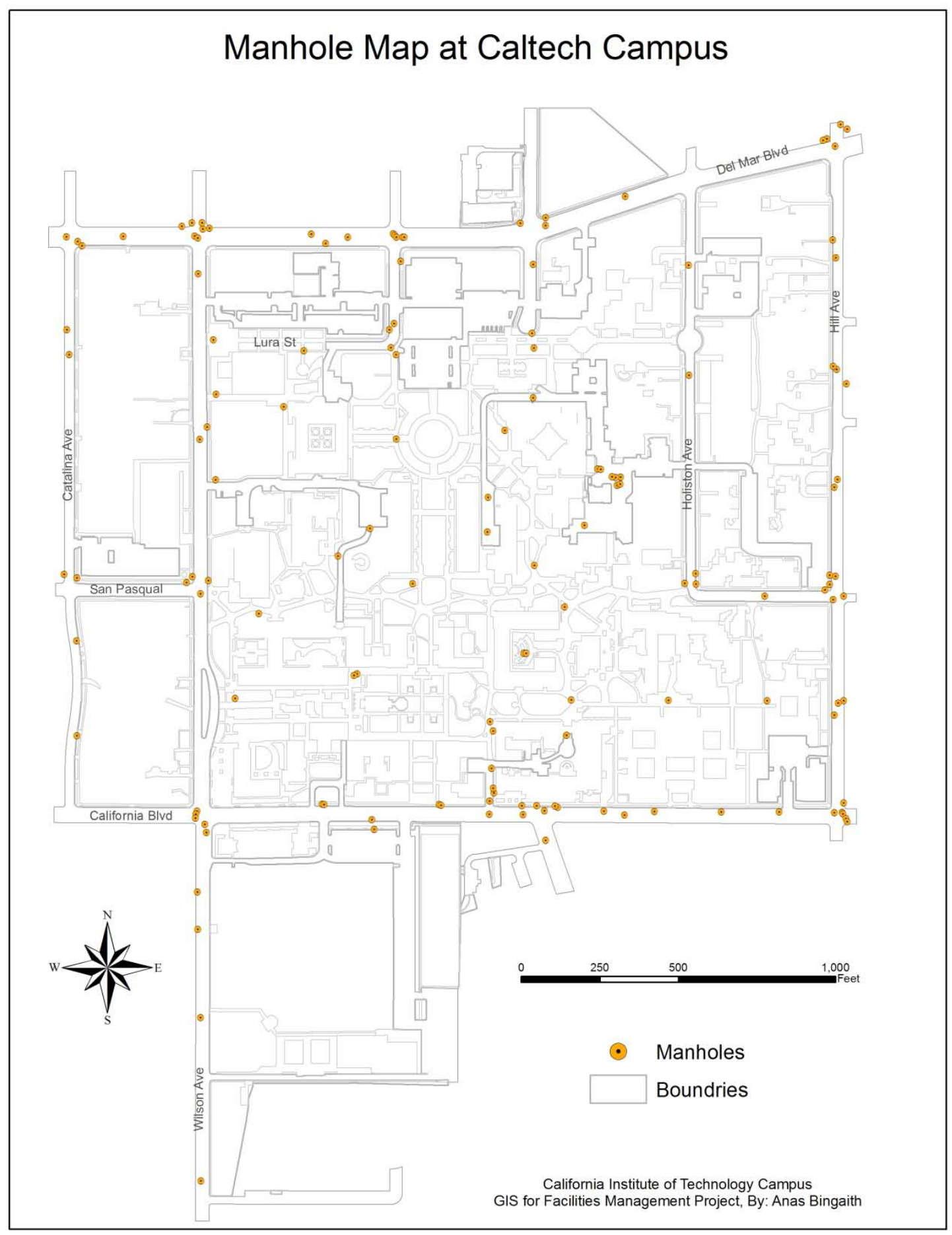


Fire Hydrant Map at Caltech Campus

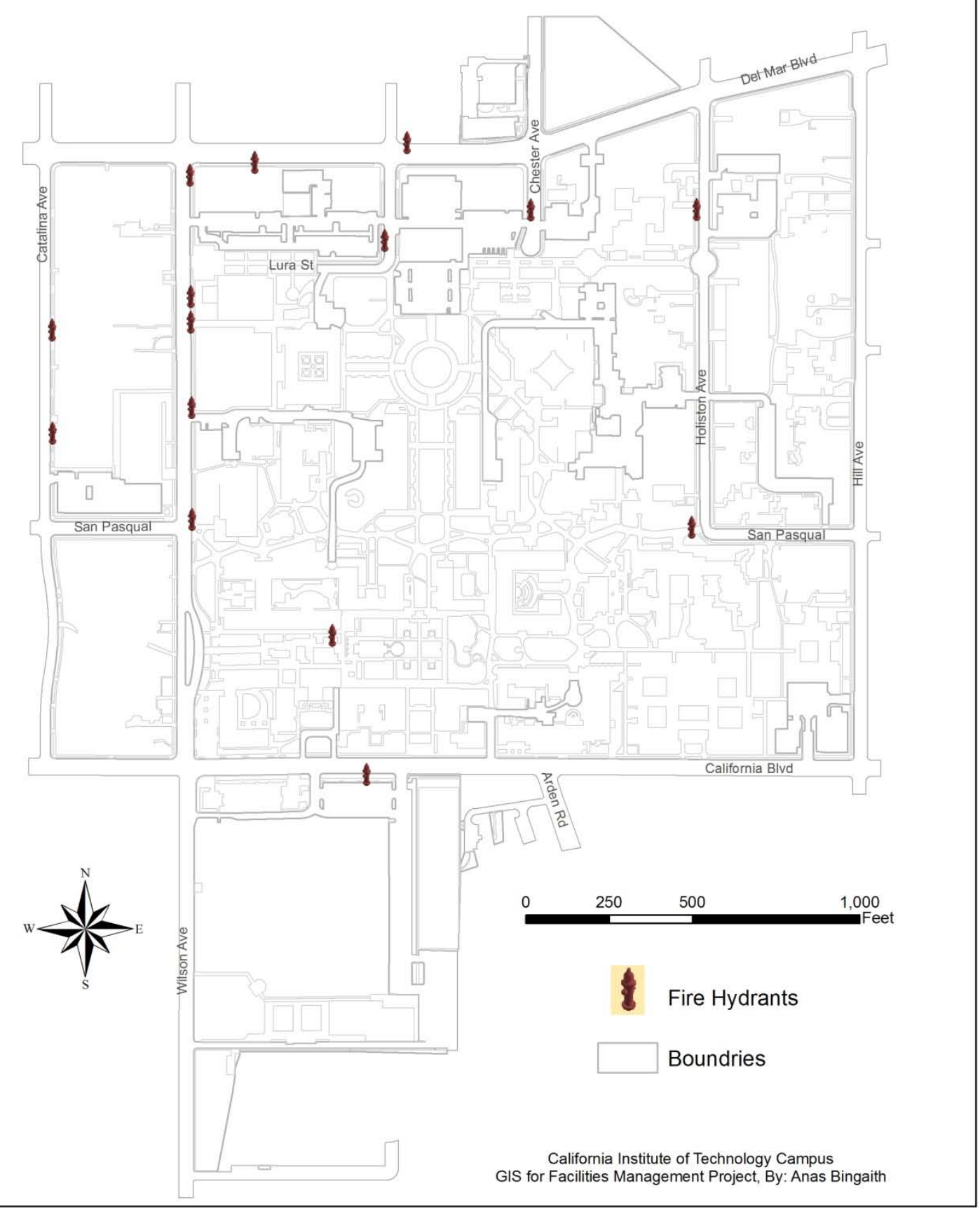




\section{Emergency Telephone Map at Caltech Campus}

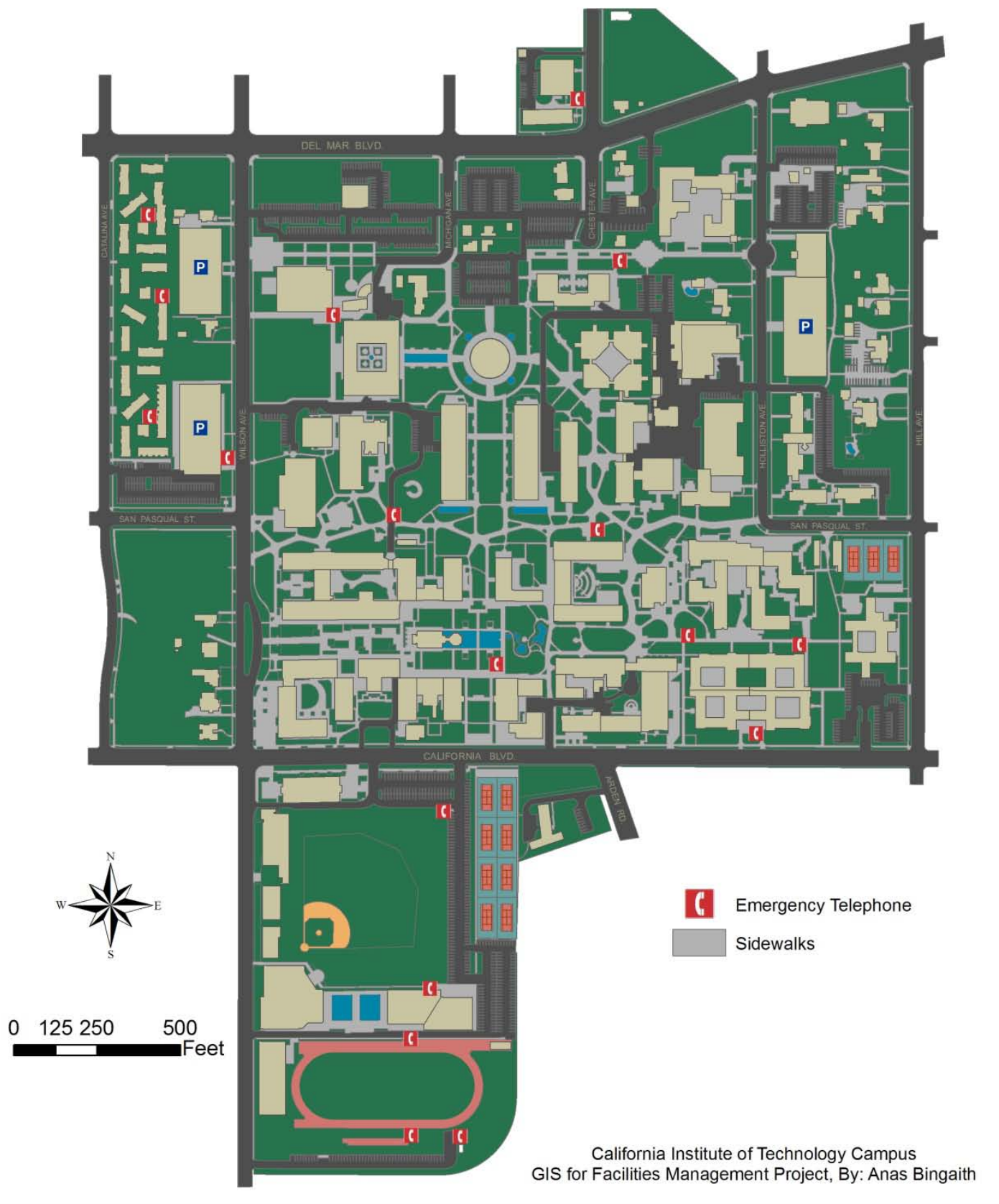





\section{Appendix C. Underground Facility Campus Maps}

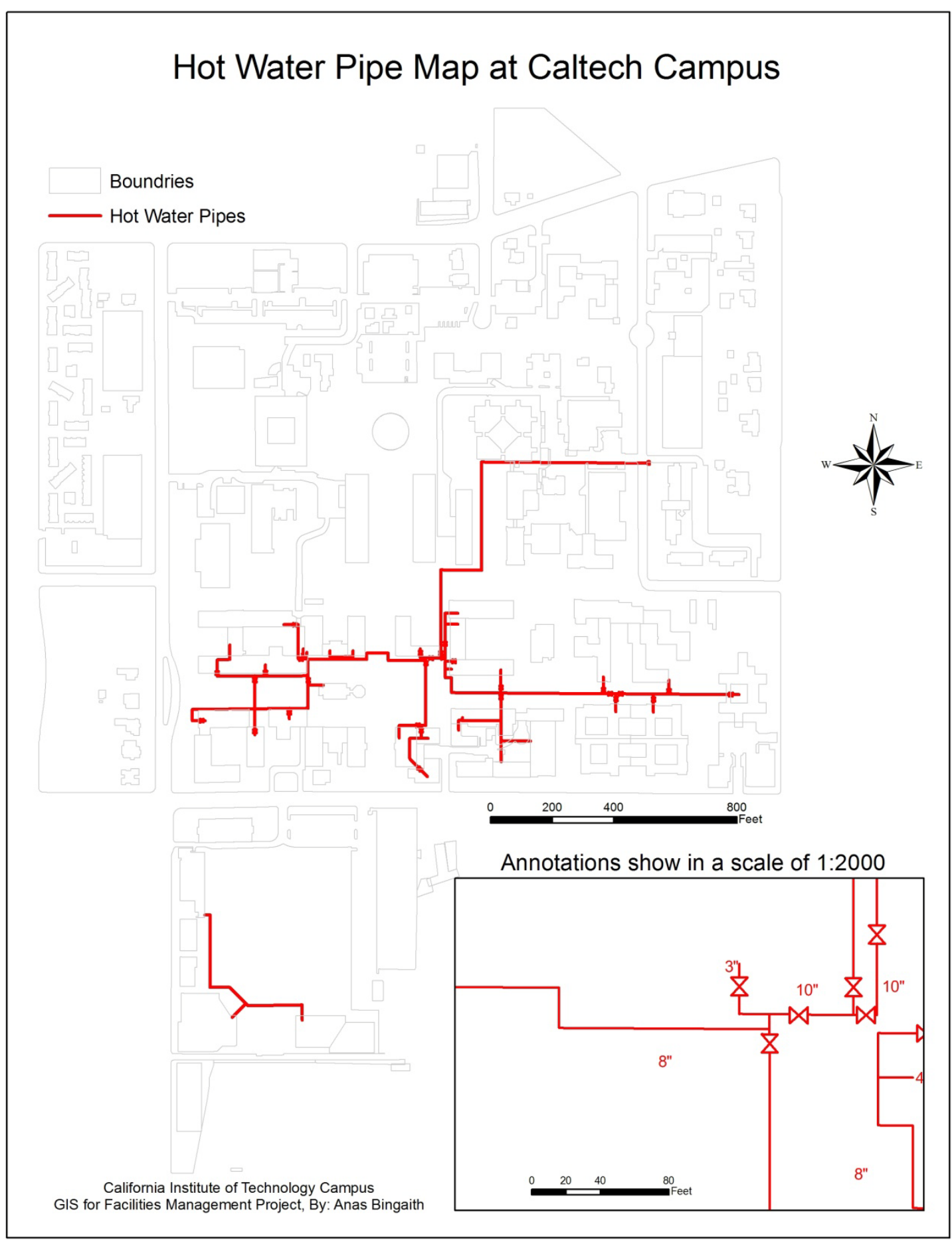




\section{Demonized Water Pipe Map at Caltech Campus}

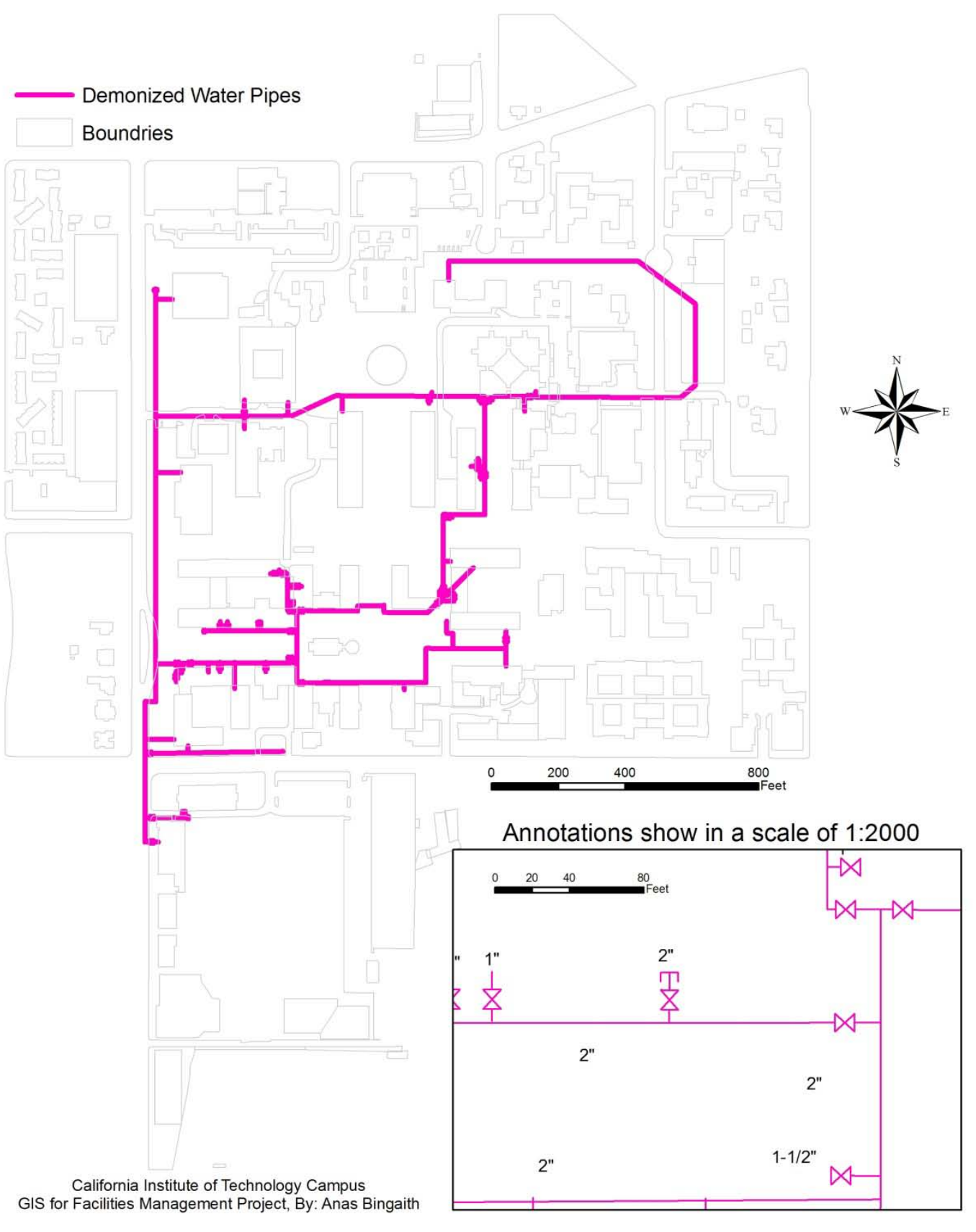




\section{Domestic Water Pipe Map at Caltech Campus}

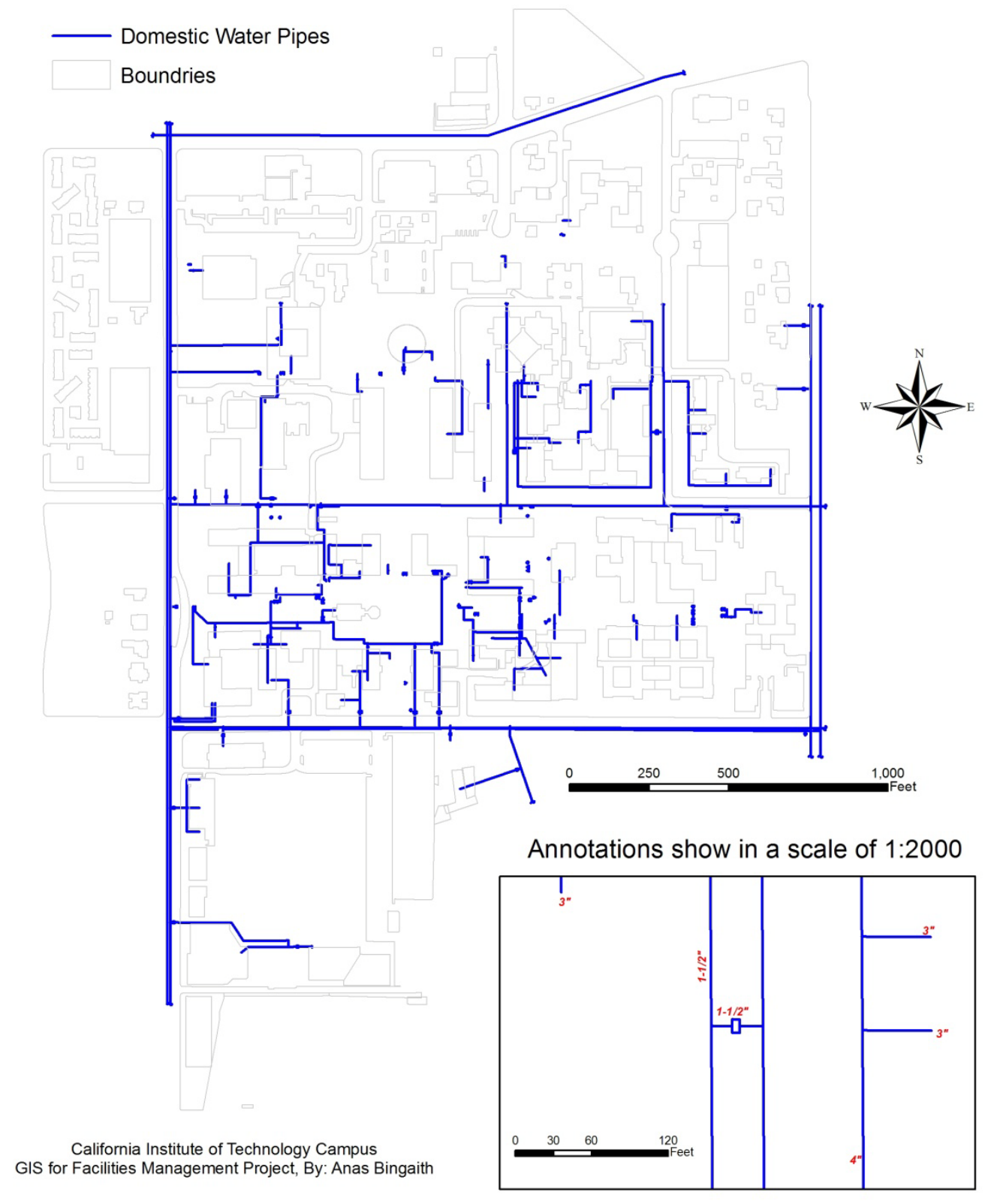




\section{Compressed Air Pipe Map at Caltech Campus}

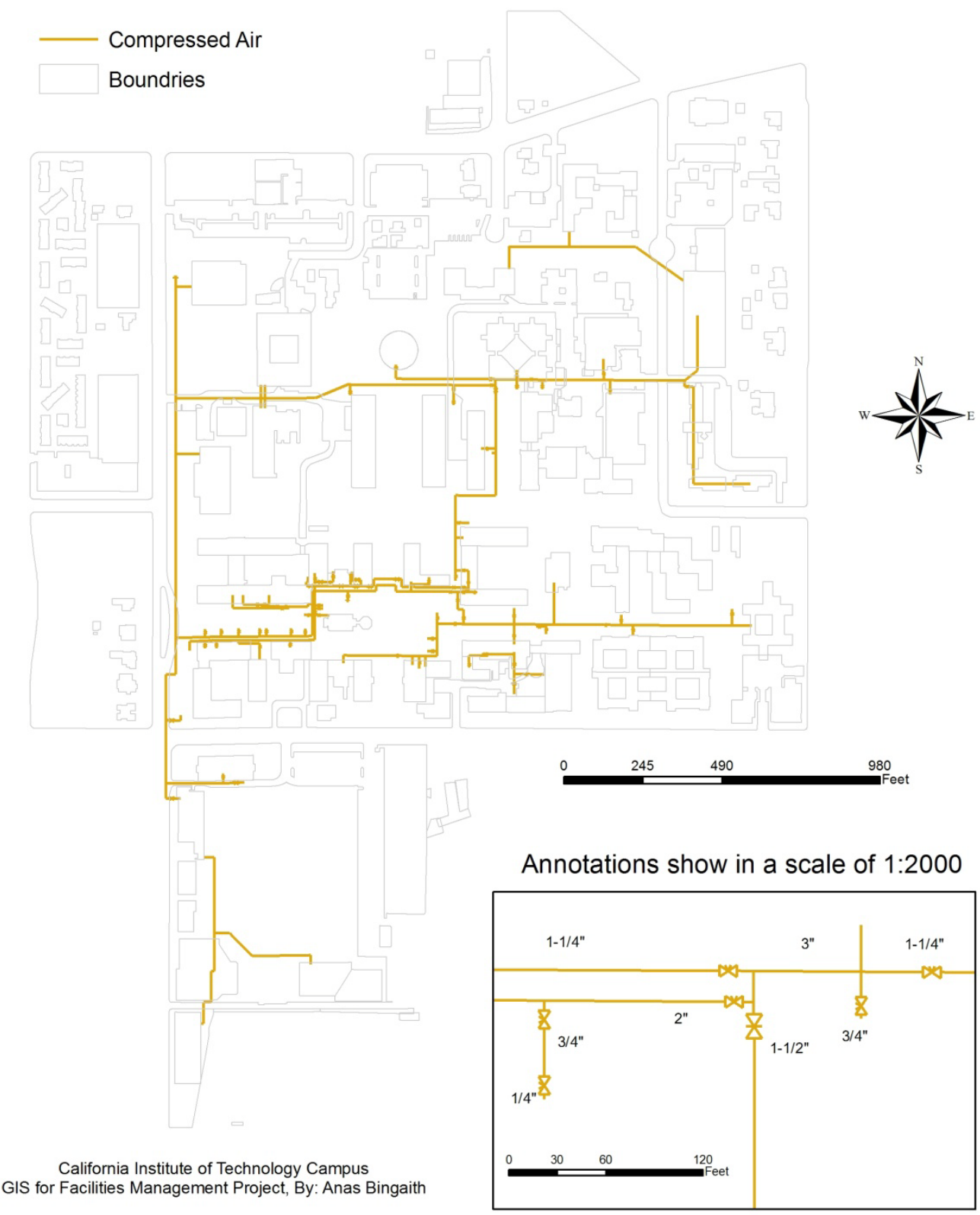




\section{Steam Pipe Map at Caltech Campus}

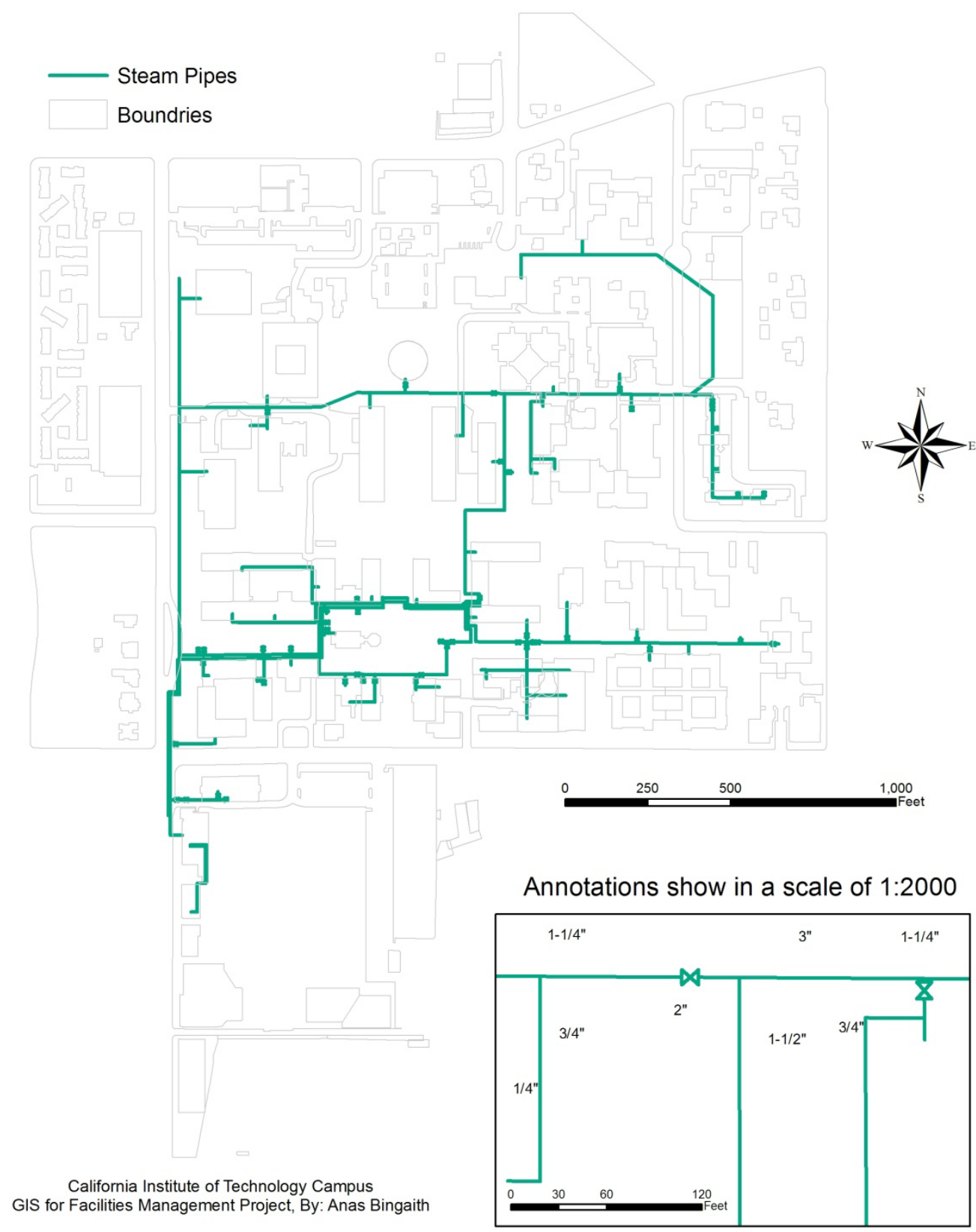




\section{Chilled Water Pipe Map at Caltech Campus}

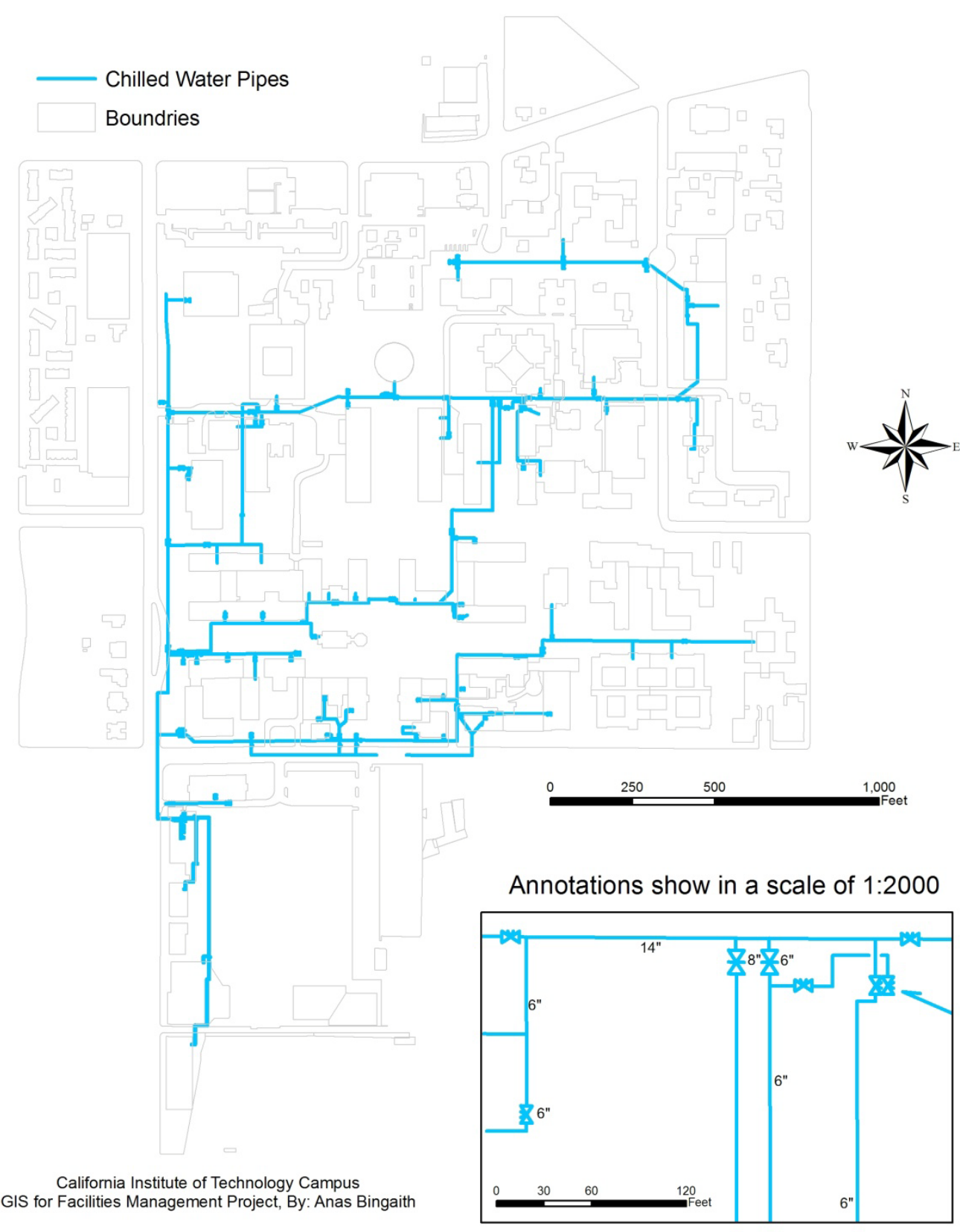




\section{Condensate Water Pipe Map at Caltech Campus}

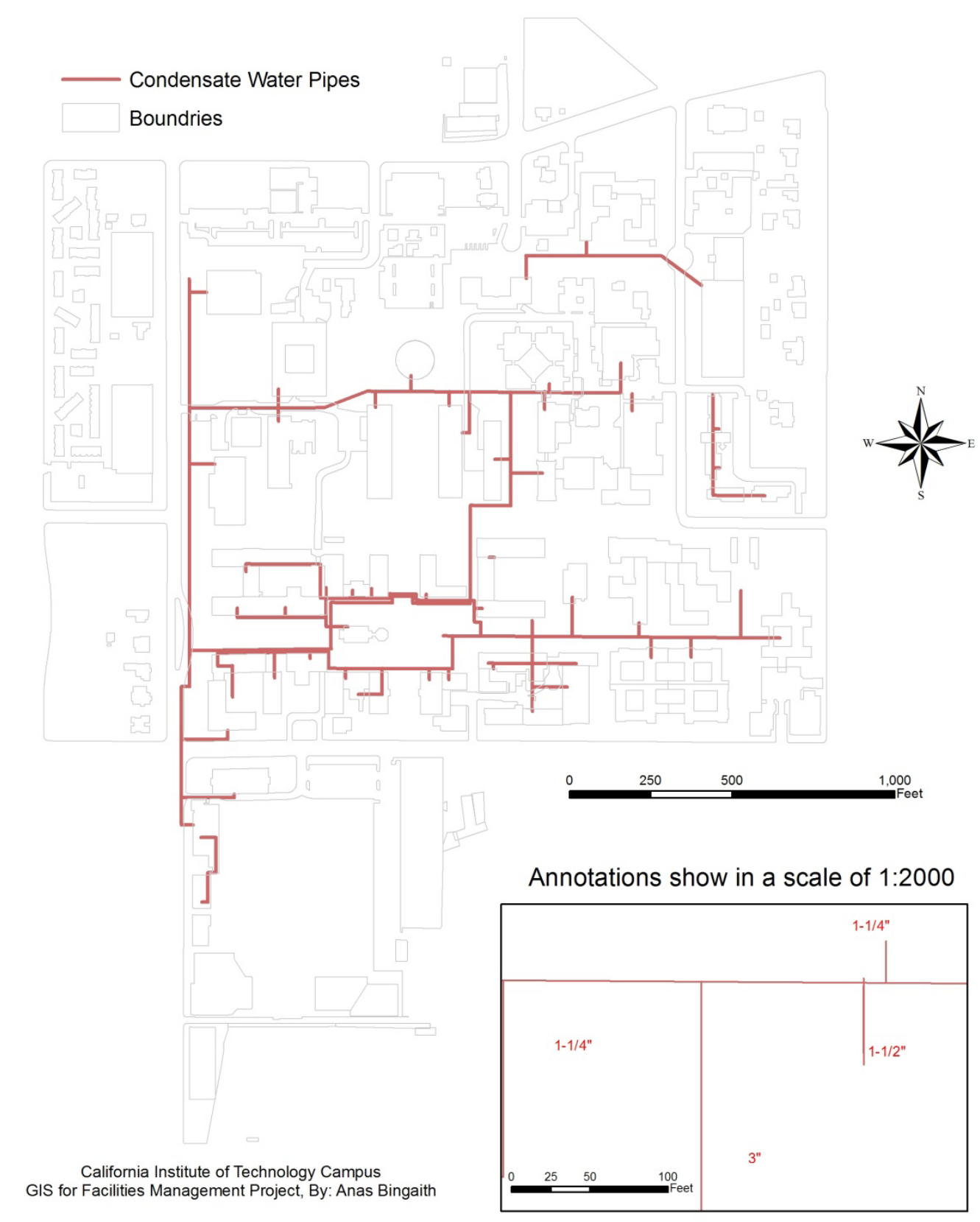




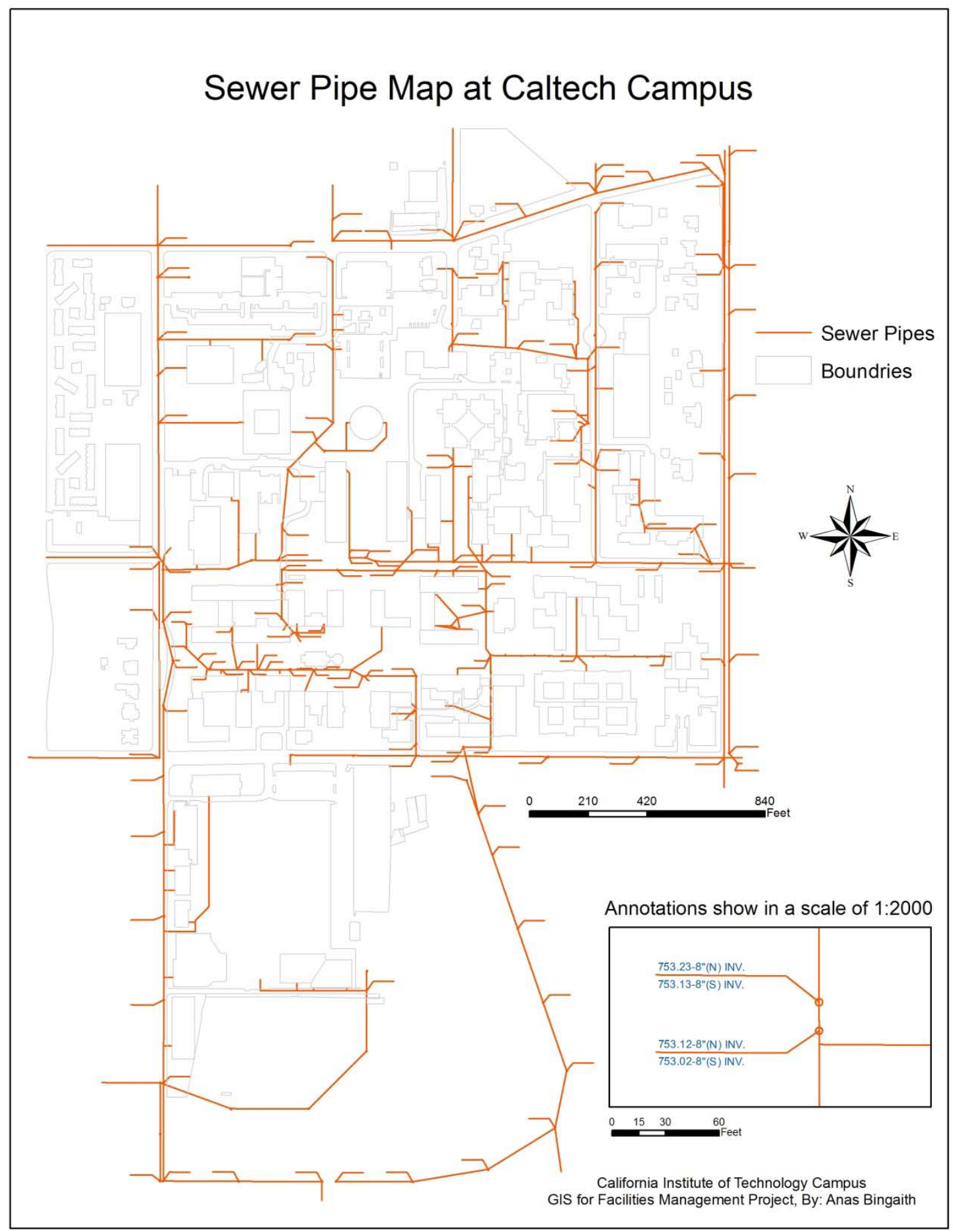




\section{Storm Water Pipe Map at Caltech Campus}

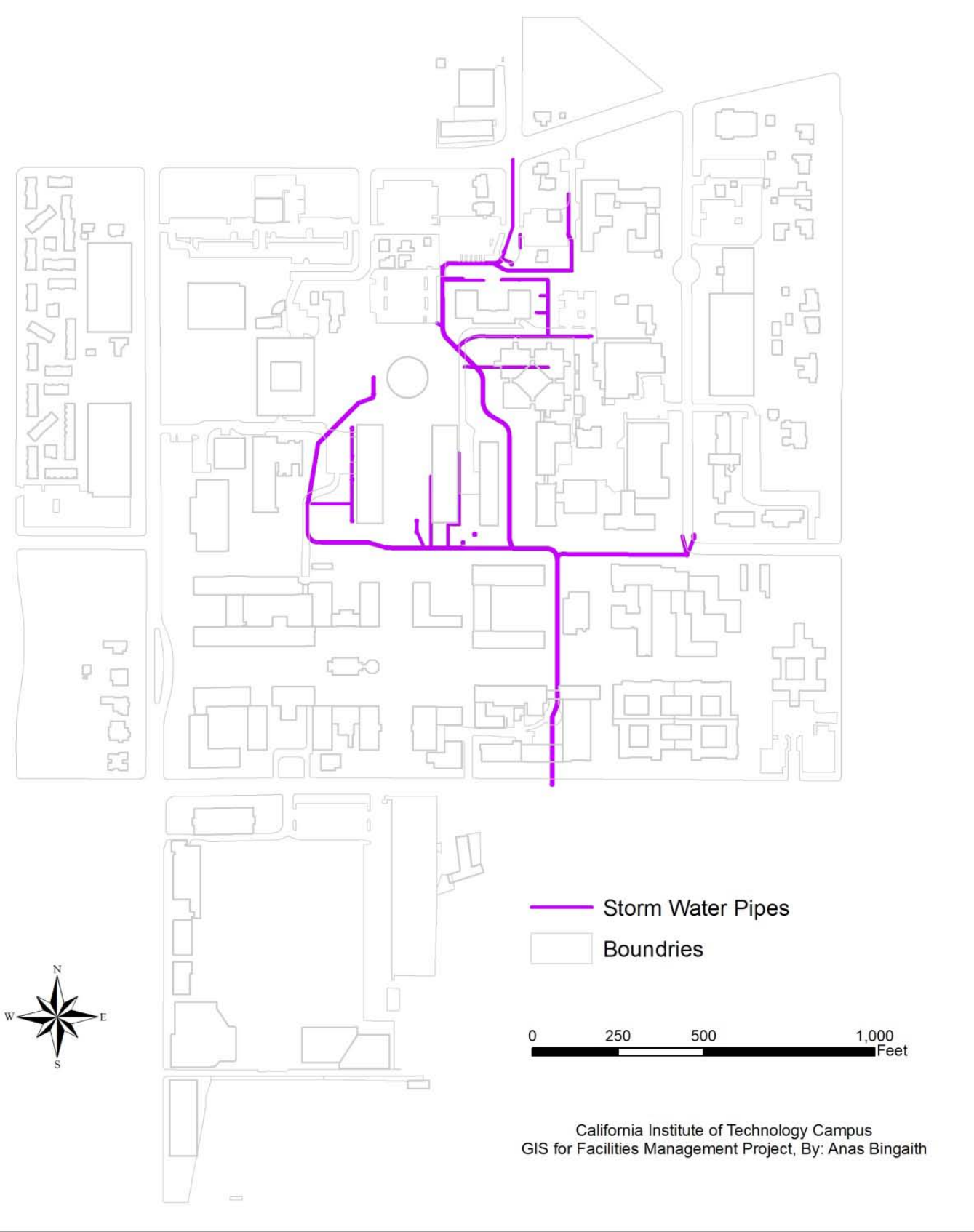




\section{Tunnel Map at Caltech Campus}

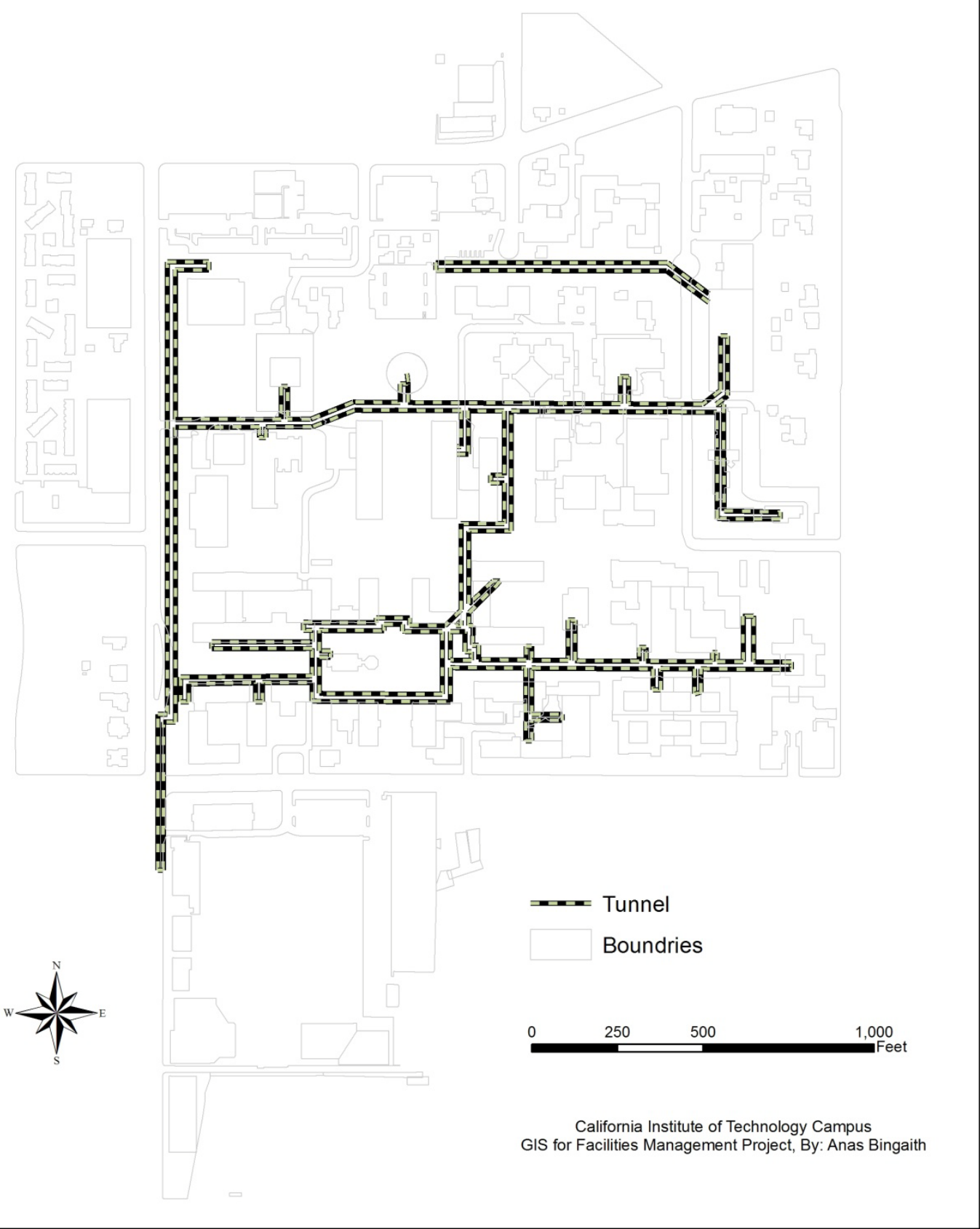

